University of New Orleans

ScholarWorks@UNO

Fall 12-17-2011

\title{
A Dynamical Study of the Evolution of Pressure Waves Propagating through a Semi-Infinite Region of Homogeneous Gas Combustion Subject to a Time-Harmonic Signal at the Boundary
}

John Eslick

University of New Orleans, drjeslick@gmail.com

Follow this and additional works at: https://scholarworks.uno.edu/td

Part of the Non-linear Dynamics Commons, Numerical Analysis and Computation Commons, and the Partial Differential Equations Commons

\section{Recommended Citation}

Eslick, John, "A Dynamical Study of the Evolution of Pressure Waves Propagating through a Semi-Infinite Region of Homogeneous Gas Combustion Subject to a Time-Harmonic Signal at the Boundary" (2011). University of New Orleans Theses and Dissertations. 1367.

https://scholarworks.uno.edu/td/1367

This Dissertation-Restricted is protected by copyright and/or related rights. It has been brought to you by ScholarWorks@UNO with permission from the rights-holder(s). You are free to use this Dissertation-Restricted in any way that is permitted by the copyright and related rights legislation that applies to your use. For other uses you need to obtain permission from the rights-holder(s) directly, unless additional rights are indicated by a Creative Commons license in the record and/or on the work itself.

This Dissertation-Restricted has been accepted for inclusion in University of New Orleans Theses and Dissertations by an authorized administrator of ScholarWorks@UNO. For more information, please contact scholarworks@uno.edu. 
A Dynamical Study of the Evolution of Pressure Waves Propagating through a Semi-Infinite Region of Homogeneous Gas Combustion Subject to a Time-Harmonic Signal at the Boundary

\author{
A Dissertation \\ Submitted to the Graduate Faculty of the \\ University of New Orleans \\ in partial fulfillment of the \\ requirements for the degree of \\ Doctor of Philosophy \\ In \\ Engineering and Applied Science \\ Physics \\ by
}

John Eslick

B.S. in Mathematics, University of New Orleans, 1997

M.S. in Mathematics, University of New Orleans, 1999

M.S. in Applied Physics, University of New Orleans, 2011

December, 2011 


\section{Acknowledgements}

I would like to thank my friends and family, especially John and Julie Guidry and my son Kory for all of their support and encouragement. I would also like to thank my advisor Dr. Puri, who spent many hours providing the necessary guidance during my graduate career. Finally, I would

like to thank my committee members, Dr. Guccione, Dr. Ioup, Dr. Malkinski and Dr. Murphy for their valuable assistance. 


\section{Table of Contents}

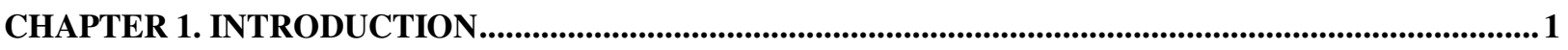

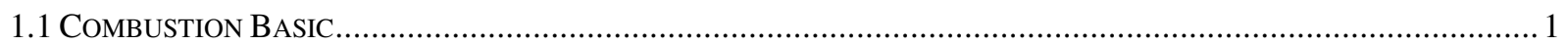

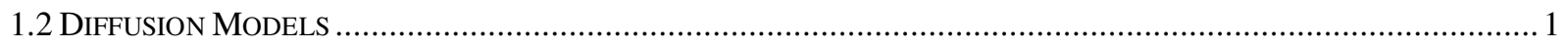

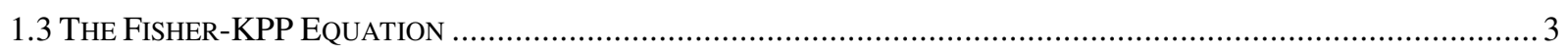

1.4 More Realistic Models of Diffusion: The Maxwell-Cattaneo Model and the GNiI Flux Law ........ 4

1.5 A Dynamical Model of Combustion: A Microscopic APProaCh........................................................ 6

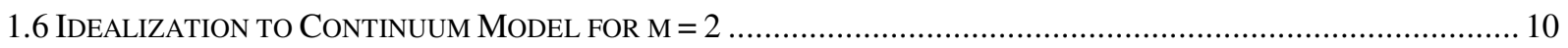

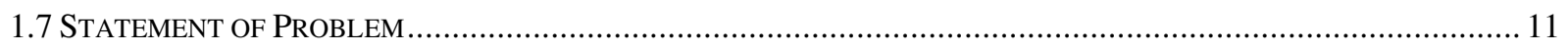

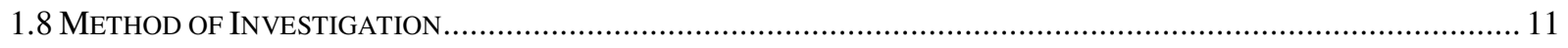

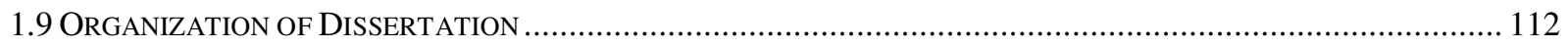

CHAPTER 2. DETERMINING THE ZEROTH-ORDER TERM AND THE FIRST AND SECOND-ORDER

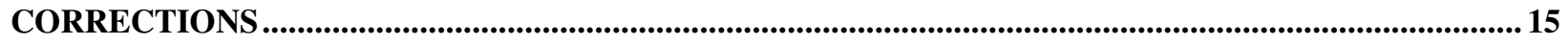

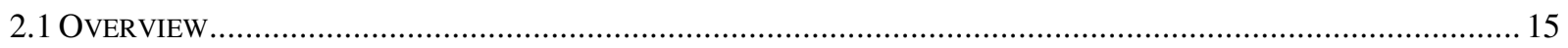

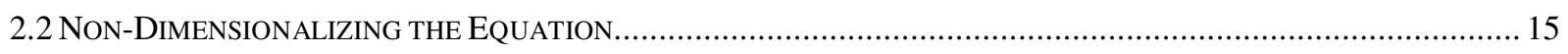

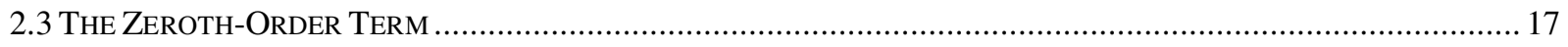

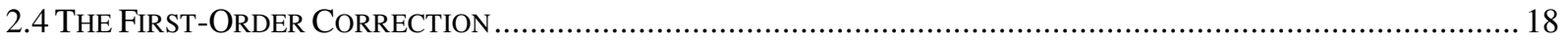

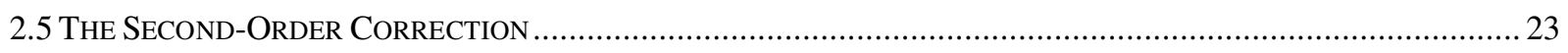

CHAPTER 3. DISPERSION RELATIONS AND PHASE SPEEDS................................................................29

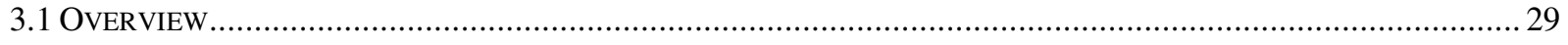

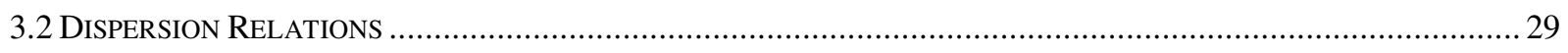

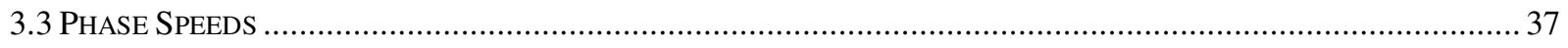

CHAPTER 4. DETERMINING THE LOCATION OF THE PEAK OF THE FIRST-ORDER

CORRECTION AND CONSTRAINTS ON THE PERTURBATION PARAMETER ................................... 42

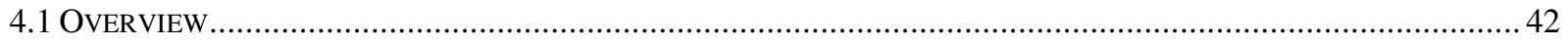

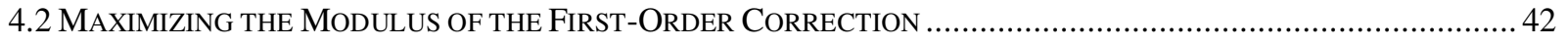


4.3 DETERMINING THE CONSTRAINRS ON THE PERTURBATION PARAMETER …..................................................... 50

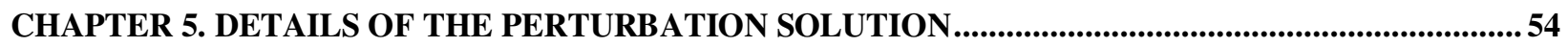

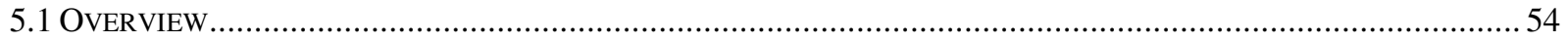

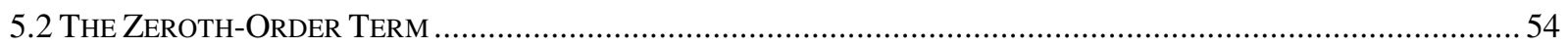

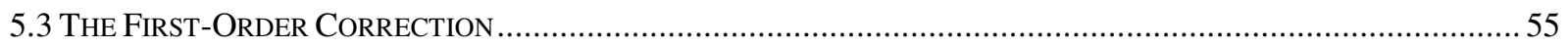

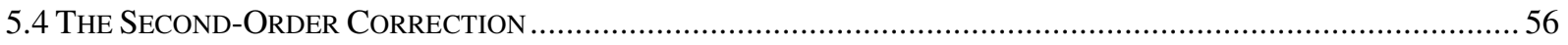

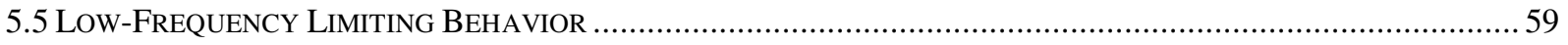

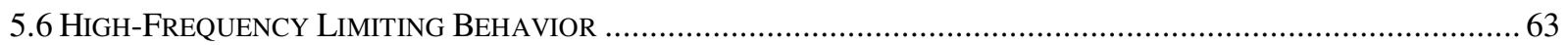

CHAPTER 6. COMPARISON OF THE PERTURBATION SOLUTION WITH THE FINITE-

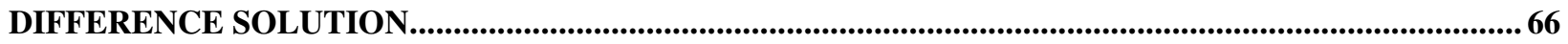

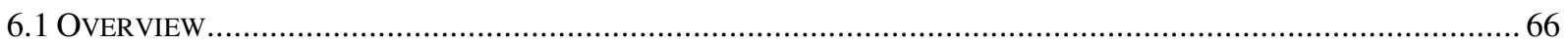

6.2 Demonstrating the Convergence of the Finite-Difference Scheme OVer One Quarter-

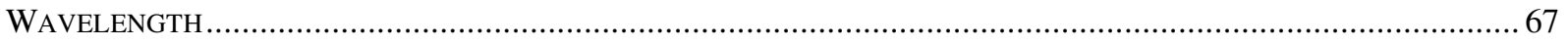

6.3 Determining the Position of the Right Boundary such that the Perturbation Solution DeCays to

$10 \%$

6.4 USing the Finite-Difference Solution to Establish the VAlidity of the Perturbation Solution .. 69

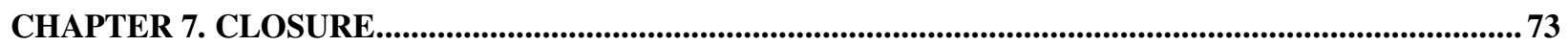

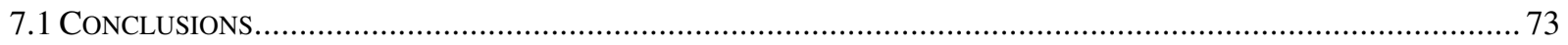

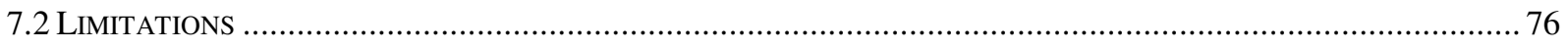

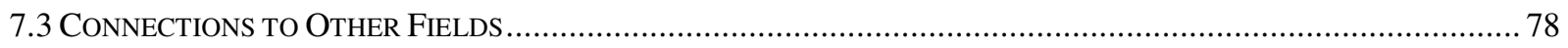

APPENDIX A. MATHEMATICA CODE FOR THE FINITE-DIFFERENCE CALCULATION.................... 79

APPENDIX B. MATHEMATICA CODE FOR COMPUTING THE LOCATION OF THE PEAK OF THE

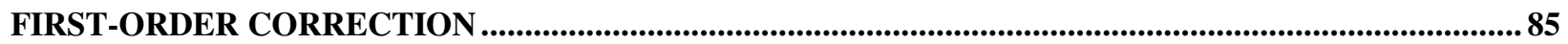

VITA 


\begin{abstract}
In this dissertation, the evolution of a pressure wave driven by a harmonic signal on the boundary during gas combustion is studied. The problem is modeled by a nonlinear, hyperbolic partial differential equation. Steady-state behavior is investigated using the perturbation method to ensure that enough time has passed for any transient effects to have dissipated. The zeroth, first and second-order perturbation solutions are obtained and their moduli are plotted against frequency. It is seen that the first and second-order corrections have unique maxima that shift to the right as the frequency decreases and to the left as the frequency increases. Dispersion relations are determined and their limiting behavior investigated in the low and high frequency regimes. It is seen that for low frequencies, the medium assumes a diffusive-like nature. However, for high frequencies the medium behaves similarly to one exhibiting relaxation. The phase speed is determined and its limiting behavior examined. For low frequencies, the phase speed is approximately equal to $\sqrt{\bar{\omega} /(n+1)}$ and for high frequencies, it behaves as $1 /(\mathrm{n}+1)$, where $n$ is the mode number. Additionally, a maximum allowable value of the perturbation parameter, $\varepsilon=0.8$, is determined that ensures boundedness of the solution. The location of the peak of the first-order correction, $\bar{x}_{\max }$, as a function of frequency is determined and is seen to approach the limiting value of $0.828 / \sqrt{\bar{\omega}}$ as the frequency tends to zero and the constant value of $2 \ln 2$ as the frequency tends to infinity. Analytic expressions are obtained for the approximate general perturbation solution in the low and high-frequency regimes and are plotted together with the perturbation solution in the corresponding frequency regimes, where the agreement is seen to be excellent. Finally, the solution obtained from the perturbation method is compared with the long-time solution obtained by the finite-difference scheme; again, ensuring that the transient effects have dissipated. Since the finite-difference scheme requires a right boundary, its location is chosen so that the wave dissipates in amplitude enough so that any reflections from the boundary will be negligible. The perturbation solution and the finitedifference solution are found to be in excellent agreement. Thus, the validity of the perturbation method is established.
\end{abstract}

Keywords: nonlinear hyperbolic equation, gas combustion, perturbation, non-standard finitedifference, pressure 


\section{Chapter 1. Introduction}

\subsection{Combustion Basics}

Combustion is a sequence of exothermic reactions between a fuel and an oxidant resulting in the liberation of heat and the conversion of chemical species. The fuel can either be a solid, liquid, or gas. Combustion phenomena have been studied kinematically as reaction-diffusion (RD) processes. Some physical examples of combustion phenomena modeled as RD processes are: the study of wild land fires modeled as combustion in two dimensional fuel beds [3, 4], the spread of fire through a porous fuel bed [9], the pyrolysis and combustion of bark [6] and ignition criteria pulverized coal flow [33]. Source terms corresponding to various diffusion models are presented below.

\subsection{Diffusion Models}

Combustion has been studied as a diffusion process from a kinematic point-of-view. The kinematic models arise using the heat (diffusion) equation, flux conditions, and conservation laws. Fick's law, which relates the mass flux $\mathbf{q}$ to the density gradient $\nabla \rho$ via $\mathbf{q}=-D \nabla \rho$, when combined with the continuity equation (mass conservation equation), $\frac{\partial \rho}{\partial t}+\nabla \cdot \mathbf{q}=S(\mathbf{r})$, where $\mathbf{r}$ is the position vector, leads to the general scalar RD equation $[1,3]$

$$
\frac{\partial \rho}{\partial t}-D \frac{\partial^{2} \rho}{\partial x^{2}}=S(\rho, x, t)
$$


a parabolic partial differential equation $[1,8,10]$. Here, the diffusivity $D$ is constant for media and the source term $S(\rho, x, t)$ accounts for the processes of production and annihilation. There are several forms for the source term that of particular importance to researchers in many fields.

Some examples of source terms are listed below.

- The Arrhenius law [32], which states that the reaction-rate constant $k$ is empirically related to the temperature by $k=A e^{-\frac{E}{R_{0} T}}$, where $A$ and $E$ are the frequency factor and activation energy, respectively, and $R_{0}$ is the universal gas constant [32]. Still other models include a convection term. This convection term can be combined with the Arrhenius law.

- This approach has been applied by considering a linear convection term coupled with the Arrhenius law: $-B\left(n-n_{0}\right)+A e^{-\frac{E}{R_{0} T}}$. Here, $B$ is positive and $n$ is the concentration.

- In $[3,4]$, the temperature spread in a two dimensional fuel bed is modeled by incorporating the linear convection term, but replacing the Arrhenius law with $c e^{-\alpha t} H(t)$, where $c$ and $\alpha$ are positive constants and $H(t)$, is the step function, i.e., $S \sim-b\left(n-n_{0}\right)+c e^{-\alpha t} H(t)$.

- If $S$ is a constant function, then the telegrapher equation results. Derivations of the telegrapher equation generally assumes a constant signal propagation speed. However, in [20], the model is generalized to allow for variable propagation speeds.

- If $S=\alpha \rho\left(1-\rho / \rho_{s}\right)$, the Pearl-Verhulst growth law, the Fisher-KPP equation results (see below). Here the positive constants $\alpha$ and $\rho_{s}$ are the growth-rate 
coefficient and saturation density, respectively. The Pearl-Verhulst law is used in some diffusive models of population growth [30, 31].

- $S=\alpha \rho\left[1-\left(\rho / \rho_{s}\right)^{2}\right]$ is called the Fisher law.

- $S=\alpha \rho_{s} \rho^{m}\left[1-\left(\rho / \rho_{s}\right)^{m}\right],(m=2,3)$ is called the mth-order Fisher law. The case $m=2$ is also called the Zeldovich law [34].

- $S=\varepsilon \sigma\left(u_{0}{ }^{4}-u^{4}\right),(0<\varepsilon<1)$ is called the Stefan-Boltzmann law [5, 29]. Here, $\varepsilon$ is the emissivity and the positive constants $\sigma$ and $u_{0}$ are the Stefan-Boltzmann constant and surrounding temperature, respectively.

\subsection{The Fisher-KPP Equation}

Of premium importance is when the source term is given by the Pearl-Verhulst law resulting in the Fisher-KPP equation, owing its name to Fisher [11] and Kolmogoroff, et al. [19], who independently investigated the behavior of the spread of an advantageous gene in a population through random mating by modeling it as a wave-like, as opposed to a diffusive process. The Fisher-KPP equation is a nonlinear, scalar RD equation with a Pearl-Verhulst growth law. The Fisher-KPP equation is obtained by combining the balance law for the species or mass with the constitutive relation known as Fick's law, which states that the mass flux is proportional to the negative of the temperature gradient: $\mathbf{q}=-D \nabla \rho$. The positive constant $D$ is called the diffusivity and depends on the material properties. The Fisher-KPP equation occurs in many areas other than the life sciences, such as the physical sciences and the social sciences $[2,28]$. 


\subsection{More Realistic Models of Diffusion: The Maxwell-Cattaneo Model and the GNII Flux Law}

The diffusion equation obtained in Section 1.2 implies that a material diffusion at any point in a material will be felt instantaneously. This unphysical feature was first discovered as a "paradox of heat conduction (diffusion)" by Maxwell in 1867 and researchers in the theory of heat conduction, where Fick's law is known as Fourier's law, found it necessary to modify it, eliminating the unphysical result of instantaneous transmission of information across the system $[15,30]$. It is most evident under low temperatures/concentrations and high heat-flux conditions. To avoid the paradox of diffusion, we consider the Maxwell-Cattaneo (MC) model, which predicts that diffusion results from damped temperature waves that propagate with finite speed $[12,20]$, by including a relaxation rate $\tau_{0}$ in the flux condition. By defining the operator $\widehat{\mathbf{L}} \equiv 1+\tau_{0} \frac{\partial}{\partial t}$, the MC model can be written in operator form as

$$
\widehat{\mathbf{L}} \mathbf{q}=-D \nabla \rho
$$

Applying the operator $\widehat{\mathbf{L}}$ to the continuity equation $\frac{\partial \rho}{\partial t}+\nabla \cdot \mathbf{q}=S(\rho)$, where the function $S(\rho)$ is, in general, nonlinear gives

$$
\widehat{\mathbf{L}}\left[\frac{\partial \rho}{\partial t}+\nabla \cdot \mathbf{q}\right]=\widehat{\mathbf{L}}[S(\rho)]
$$


or, expanding and using Equation (1.4.1),

$$
\frac{\partial \rho}{\partial t}+\tau_{0} \frac{\partial^{2} \rho}{\partial t^{2}}-D \nabla^{2} \rho=S(\rho)+\tau_{0} \frac{\partial S}{\partial t}
$$

a linear, damped wave equation with a source.

Note that in the limit, $\tau_{0} \rightarrow 0$, Equation (1.4.3) reduces to the (static diffusion)

equation. Then, for $\tau_{0}>0$, we have, upon dividing both sides of Equation (1.4.3) by $\tau_{0}$,

$$
\tau_{0}^{-1} \frac{\partial \rho}{\partial t}+\frac{\partial^{2} \rho}{\partial t^{2}}-D \tau_{0}^{-1} \nabla^{2} \rho=\tau_{0}{ }^{-1} S(\rho)+\frac{\partial S}{\partial t}
$$

Now, letting $\tau_{0} \rightarrow \infty$ and assuming the quantity $D \tau_{0}{ }^{-1}$ remains bounded, we obtain a (propagation/combustion) equation

$$
\frac{\partial^{2} \rho}{\partial t^{2}}-c_{\infty}^{2} \nabla^{2} \rho=\frac{\partial S}{\partial t}
$$

where the quantity $D \tau_{0}{ }^{-1}=c_{\infty}{ }^{2}$ is the square of the speed of the wave. In the limit of infinite relaxation time, the MC model corresponds to the GNII flux model. GNII is the second of three versions of a theory of thermoelasticity developed in the 1990s by Green and Nagdi [13, 14]. Recently, the transient behavior of GNII model has been studied by Jordan and Puri [18]. When the source term is the Pearl-Verhulst law, we have

$$
\frac{\partial S}{\partial t}=\frac{\partial}{\partial t}\left[\alpha \rho\left(1-\frac{\rho}{\rho_{s}}\right)\right]=\alpha\left[\left(1-\frac{\rho}{\rho_{s}}\right) \frac{\partial \rho}{\partial t}-\frac{\rho}{\rho_{s}} \frac{\partial \rho}{\partial t}\right]=\alpha\left(1-\frac{2 \rho}{\rho_{s}}\right) \frac{\partial \rho}{\partial t}
$$


Thus, Equation (1.4.5) becomes

$$
\frac{\partial^{2} \rho}{\partial t^{2}}-c_{\infty}^{2} \nabla^{2} \rho=\alpha\left(1-\frac{2 \rho}{\rho_{s}}\right) \frac{\partial \rho}{\partial t},
$$

or

$$
\frac{\partial^{2} \rho}{\partial t^{2}}-\alpha\left(1-\frac{2 \rho}{\rho_{s}}\right) \frac{\partial \rho}{\partial t}-c_{\infty}{ }^{2} \nabla^{2} \rho=0
$$

\subsection{A Dynamical Model of Combustion: A Microscopic Approach}

Most models of combustion are kinematic models. However, Rosen [24] studied a dynamical model of the fluid flow and the mechanism by which liquid propellant droplets are entrained. An equation for pressure waves in the combustion field is developed, which is then used to analyze the stability of the pressure waves. To simplify the analysis, only processes satisfying the following conditions are considered:

1. The analysis is confined to one spatial dimension.

2. Only fuels for which the average Reynold's number is small were studied. This ensures entrainment of the droplets in the burned gas flow.

3. Viscous effects of the fluid in the longitudinal direction are assumed negligible.

4. Combustion of the droplets is governed by one-step rate controlling physical and chemical processes.

5. The local density of the burned gas is related to the local pressure algebraically. The derivation begins with four governing equations: the conservation laws for mass and momentum, and the production rate and process relation for the burned gas: 


$$
\begin{gathered}
\frac{\partial \rho}{\partial t}+\frac{\partial(\rho u)}{\partial x}=0 \\
\frac{\partial u}{\partial t}+u \frac{\partial u}{\partial x}+\frac{1}{\rho} \frac{\partial P}{\partial x}=0 \\
\frac{\partial \omega}{\partial t}+u \frac{\partial \omega}{\partial x}=f(\omega, P ; \text { fluid element }) \\
\omega \rho=g(P)
\end{gathered}
$$

where $\rho, u, P$ and $\omega$ are the mixture density, mixture velocity, pressure and weight fraction of the burned gas, respectively. The burning rate function $f$ may depend parametrically on the fluid element. As a result, both homogeneous and heterogeneous combustion is admitted. For this investigation, it is assumed that the burning rate function and process relation to have the form

$$
\begin{array}{lr}
f(\omega, P ; \psi)=P^{m-1} \phi(\omega ; \psi) & m \geq 1 \\
g(P)=(-b P+a)^{-1} & a, b>0
\end{array},
$$

where $\psi$ represents the dependency on the fluid element and is a Lagrangian coordinate defined by $d \psi=\rho d x-\rho u d t, m$ is the effective order of the physical or chemical process controlling the combustion of the droplets, and the constants $a, b$ give the "tangent-gas" behavior [7] and relate to average conditions in the burned gas and $\phi$ is nonnegative and vanishes for all fluid elements at the initial and final states of combustion. For practical cases, the constant $m$ ranges between 1 and 3 .

Upon utilizing the Lagrange coordinate, manipulating the resulting equations and using the assumed forms for $f$ and $g$, the governing equation for pressure waves is obtained

$$
\omega a \frac{\partial^{2} P}{\partial t^{2}}+\phi\left[b(m+1) P^{m-1}-a(m-1) P^{m-2}\right] \frac{\partial P}{\partial t}+\phi \frac{\partial \phi}{\partial \omega} P^{2 m-2}(a P-b)-\frac{\partial^{2} P}{\partial \psi^{2}}=0
$$


Outside the region of active combustion, after averaging over $\omega$ as $\omega$ varies from $\omega_{0}$ to 1

(Outside the region of combustion, $\omega$ is either $\omega_{0}$ or 1.), and holding $P$ and its derivatives fixed, the following equation results

$$
\frac{1}{2}\left(1+\omega_{0}\right) a \frac{\partial^{2} P}{\partial t^{2}}+\bar{\phi}\left[b(m+1) P^{m-1}-a(m-1) P^{m-2}\right] \frac{\partial P}{\partial t}-\frac{\partial^{2} P}{\partial \psi^{2}}=0 .
$$

Here, the middle term is due to the interaction of the pressure waves with the combustion process, i.e., a "friction term", with the quantity $\bar{\phi}\left[b(m+1) P^{m-1}-a(m-1) P^{m-2}\right]$ being the "friction coefficient". The pressure waves are only stable if and only if this quantity is positive. This results in the necessary and sufficient condition for local stability of pressure waves which is given by

$$
\frac{b P_{c}}{a}>\frac{m-1}{m+1}
$$

where $P_{c}$ is the average chamber pressure. The effective polytropic index for the burned gas at chamber pressure is defined as

$$
\left.\kappa \equiv \frac{d \ln P}{d \ln (\omega \rho)}\right|_{P=P_{c}}=\frac{a}{b P_{c}}-1,
$$

where a polytropic gas is a gas such that there exists a constant, the polytropic index, $k$, such that $P V^{k}=$ constant, where $1.0<k \leq 1.4$. 
The validity of the RHS of Equation (1.5.3) can be demonstrated using the last equation in (1.5.1) for the process relation of the burned gas. Taking logarithms of both sides, we have, using the second equation in (1.5.2),

$$
\ln (\omega \rho)=-\ln (-b P+a)
$$

Differentiating Equation (1.5.4) with respect to $P$ gives

$$
\frac{d \ln (\omega \rho)}{d P}=-\frac{d \ln (-b P+a)}{d P}=\frac{b}{-b P+a}
$$

Then, from Equations (1.5.4) and (1.5.5), we have

$$
\frac{d \ln P}{d \ln (\omega \rho)}=\frac{d \ln P}{d P} \cdot \frac{d P}{d \ln (\omega \rho)}=\frac{1}{P} \cdot \frac{-b P+a}{b}=-1+\frac{a}{b P}
$$

Evaluating Equation (1.5.6) at $P_{c}$ gives the RHS of Equation (1.5.3). In terms of the effective polytropic index, the pressure waves are stable if and only if

$$
m<1+\frac{2}{\kappa} .
$$

To relate $\varepsilon$ and $\kappa$, we recall that $\varepsilon=b P_{a} / a$. Inverting, multiplying and dividing by $P_{c}$ and using Equation (1.5.3), we have

$$
\frac{1}{\varepsilon}=(\kappa+1) \cdot \frac{P_{c}}{P_{a}}
$$




\subsection{Idealization to Continuum Model for $m=2$}

This section deals with the macroscopic formulation of a dynamical model of gas combustion as discussed in Section 1.5 [25]. Specifically, the simplest nonlinearity is considered in the "friction term" (corresponding to $m=2$ ) of the hyperbolic pressure wave equation.

By combining the conservation laws for mass and momentum, and assuming that the excess local burning rate depends on the local pressure parabolically, and since the excess local burning rate is given by the material derivative of a thermodynamic quantity that is linear in the pressure and specific volume of the gas, mathematically the fifth assumption in Section 1.5 is given by

$$
D_{t}\left(P+\frac{f^{2}}{\rho}\right)=a\left(P-P_{c}\right)+b\left(P-P_{c}\right)^{2},
$$

where $D_{t} \equiv \partial / \partial t+u \partial / \partial x$ is the material derivative. Using a Lagrange coordinate, the following hyperbolic pressure wave equation results

$$
\frac{\partial^{2} P}{\partial t^{2}}-\left[2 b\left(P-P_{c}\right)+a\right] \frac{\partial P}{\partial t}-\frac{\partial^{2} P}{\partial x^{2}}=0
$$

where $a<0$ and the quantity $\left|b\left(P-P_{c}\right) / a\right|$ is small, admitting application of perturbation techniques. We note that the equation above corresponds precisely to the $m=2$ case discussed in Section 1.6. Thus, a two-stage combustion process corresponds to the simplest type of nonlinearity, namely parabolic. 


\subsection{Statement of Problem}

We investigate the behavior of the evolution of the damped pressure waves propagating through a one-dimensional, semi-infinite spatial region in which there is uniform, homogeneous gaseous combustion [26] subject to a time-harmonic signal at the boundary. This problem is modeled by the Boundary Value Problem (BVP):

$$
\begin{array}{cc}
\text { PDE: } & \frac{\partial^{2} P}{\partial t^{2}}-\left[2 b\left(P-P_{\mathrm{c}}\right)+a\right] \cdot \frac{\partial P}{\partial t}-\frac{\partial^{2} P}{\partial x^{2}}=0 \\
\text { BC \#1: } & P(0, t)=P_{\mathrm{a}} \sin \omega t \\
\text { BC \#2: } & P(\infty, t)=0
\end{array}
$$

\subsection{Method of Investigation}

The investigation will be carried out by considering a harmonic signal that perturbs the initial state of a homogeneous gas in a cavity, such that certain conditions formulated by G. Rosen [26] and stated in Chapter 3 are satisfied, triggering combustion. The resulting pressure waves that emerge after all transient effects have dissipated will then be analyzed. Formally, we shall use perturbation analysis as outlined in [10] to solve BVP (1.7.1). However, rather than using the boundary conditions in BVP (1.7.1), we shall use assume $P$ to be complex, $\mathcal{P}$. Thus we shall instead solve the BVP:

$$
\begin{array}{lc}
\text { PDE: } & \frac{\partial^{2} \mathcal{P}}{\partial t^{2}}-\left[2 b\left(\mathcal{P}-\mathcal{P}_{\mathrm{c}}\right)+a\right] \cdot \frac{\partial \mathcal{P}}{\partial t}-\frac{\partial^{2} \mathcal{P}}{\partial x^{2}}=0 \\
\text { BC 1: } & \mathcal{P}(0, t)=\frac{i}{2} \mathcal{P}_{\mathrm{a}} e^{-i \omega t} \\
\text { BC 2: } & \mathcal{P}(\infty, t)=0
\end{array}
$$


Then, for the final solution, we shall take $P=\mathcal{P}+$ c.c., where c.c. denotes the complex conjugate.

\subsection{Organization of Dissertation}

In Chapter 2, we begin by non-dimensionalizing BVP (1.8.1). Next, we write the (nondimensional) solution as $\overline{\mathcal{P}}$, where the bar indicates that the quantity is non-dimensional. We then use the perturbation method to determine $\overline{\overline{\mathcal{P}}}$ through second-order: $\overline{\mathcal{P}_{0}}, \overline{\mathcal{P}_{1}}$ and $\overline{\mathcal{P}_{2}}$.

In Chapter 3, we first determine analytic expressions for the real and imaginary parts of the dispersion relations, which we denote by $\bar{\alpha}_{n}$ and $\bar{\beta}_{n}$, respectively. Next, we examine the low and high frequency limiting behavior of $\bar{\alpha}_{n}$ and $\bar{\beta}_{n}$ and asymptotic expressions are obtained for both limits. The phase speeds, which we denote by $\bar{v}_{\mathrm{ph}, n}$, are analyzed in a similar way.

From Figure 2, it is readily seen that the modulus of the first-order correction, $\left|\overline{\mathcal{P}_{1}}\right|$, possesses a unique maximum value. In Chapter 4 , we investigate the behavior of the location of this maximum, which we denote by $\bar{x}_{\max }$ in terms of frequency $\bar{\omega}$. First, a transcendental equation is obtained relating $\bar{x}_{\max }$ and $\bar{\omega}$. This is done by determining an analytic expression for $\left|\overline{\mathcal{P}_{1}}\right|$ and determining its spatial derivative, $d\left|\overline{\mathcal{P}_{1}}\right| / d \bar{x}$. The maximum is then a zero of the derivative. 
In Chapter 3, we examine the dispersion relations and phase speeds of the pressure wave. First, we derive expressions for $\bar{\alpha}_{n}$ and $\bar{\beta}_{n}$. Then, we investigate and obtain the low and highfrequency limits of $\bar{\alpha}_{n}$ and $\bar{\beta}_{n}$. Next, we study the behavior of the phase speeds. We derive an expression for the phase speed, denoted by $\bar{v}_{\mathrm{ph}, n}$, valid for all frequency ranges. Finally, we study the low and high-frequency limiting behavior of $\bar{v}_{\mathrm{ph}, n}$.

The location of the peak of the modulus of the first-order correction, $\bar{x}_{\max }$, and constraints on the perturbation parameter, $\varepsilon$, are investigated in Chapter 4. $\bar{x}_{\max }$ is determined first. An equation is derived giving $\bar{x}_{\max }$ as a function of frequency. Then, the low and highfrequency limiting behaviors are studied. Analytic expressions are obtained for $\bar{x}_{\max }$ in these regions.

Chapter 5 provides details of the perturbation solution. The real partial solutions $\bar{P}_{0}, \bar{P}_{1}$ and $\bar{P}_{2}$ are simplified using $\bar{P}_{n}=\overline{\mathcal{P}}_{n}+$ c.c. . Next, real analytic expressions are obtained for the low and high-frequency limits, denoted by $\bar{P}_{n}^{L F}$ and $\bar{P}_{n}{ }^{H F}$, respectively, for $n=0,1$ and 2 . These are then compared with the solution valid for all frequency ranges $\bar{P}=\bar{P}_{0}+\varepsilon \bar{P}_{1}+\varepsilon^{2} \bar{P}_{2}$.

In Chapter 6, the solution is obtained using a (non-standard) finite-difference (FD) scheme, denoted by $\bar{\rho}$, and is compared with the perturbation solution $\bar{P}$. First, the convergence of the FD solution is verified over one quarter-wavelength. Next, the position of the right boundary needed by the FD scheme, denoted by $\bar{L}$ is determined so that the amplitude of $\bar{\rho}$ decays to $10 \%$ of its value at the source of the harmonic signal by one spatial-increment (as used in the FD computation) to the left of $\bar{L}$. A similar treatment is then given to the perturbation solution $\bar{P}$. The position (denoted by $\bar{L}$ for comparison with $\bar{\rho}$ ) is determined so that the 
amplitude of $\bar{P}$ decays to $10 \%$ of its value at the source by one quarter-wavelength to the left of $\bar{L}$. The validity of the perturbation solution is then established by comparing $\bar{P}$ with $\bar{\rho}$.

Specifically, $\bar{L}$ is taken fixed at the position determined previously. Time snapshots of the evolutions of $\bar{P}$ with $\bar{\rho}$ are then plotted where the (discrete) FD solution is superimposed onto the corresponding perturbation solution. Finally, the validity of replacing the FD solution with the linear part of the perturbation solution $\bar{P}_{0}$ for locations $\bar{x}>\bar{L}$ is established by plotting $\bar{\rho}$ over the interval $(0, \bar{L})$ and the plot of $\bar{P}_{0}$ for $\bar{x}>\bar{L}$. 


\section{Chapter 2. Determining the Zeroth-Order Term and the First and}

\section{Second-Order Corrections}

\subsection{Overview}

In this chapter, we use the perturbation method to determine the approximations to the solution of BVP (1.8.1) up through second-order. In Section 2.2, we re-write BVP (1.8.1) in nondimensional form. Next, in Section 2.3, we use perturbation analysis to derive equations for the zeroth-order solution and the first two correction terms. Sections $2.4-2.5$ are devoted to determining the solutions to the equations obtained in Section 2.3.

\subsection{Non-Dimensionalizing the Equation}

We wish to re-write BVP (1.8.1) in a non-dimensional form. Let $P^{\prime}=P-P_{\mathrm{c}}$ and $\bar{P}=P^{\prime} / P_{\mathrm{a}}$. Then, the PDE in BVP (1.8.1) becomes

$$
c_{0}^{-2} \frac{\partial^{2} \bar{P}}{\partial t^{2}}-\left(2 b P_{\mathrm{a}} \bar{P}+a\right) \frac{\partial \bar{P}}{\partial t}-\frac{\partial^{2} \bar{P}}{\partial x^{2}}=0
$$

where $P_{\mathrm{a}}$ is the pressure amplitude at the left boundary of the vessel. Then, since $a<0$, we have $a=-|a|$, and in terms of the non-dimensional time $\bar{t}=t / \tau$ and the nondimensional $\bar{x}=x / L$,

$$
c_{0}^{-2} \tau^{-2} \frac{\partial^{2} \bar{P}}{\partial \bar{t}^{2}}-\frac{|a|}{\tau} \cdot\left(\frac{2 b P_{\mathrm{a}}}{|a|} \bar{P}-1\right) \frac{\partial \bar{P}}{\partial \bar{t}}-\frac{1}{L^{2}} \frac{\partial^{2} \bar{P}}{\partial \bar{x}^{2}}=0,
$$

where $\tau$ is the characteristic time and $L=c_{0} \tau$ is the characteristic length. 
Next, let $\alpha=|a| c_{0}^{2} \tau$ and $\varepsilon=2 b P_{\mathrm{a}} /|a|$. Then,

$$
\frac{\partial^{2} \bar{P}}{\partial \bar{t}^{2}}-\alpha(\varepsilon \bar{P}-1) \frac{\partial \bar{P}}{\partial \bar{t}}-\frac{\partial^{2} \bar{P}}{\partial \bar{x}^{2}}=0
$$

Finally, letting $\bar{\omega}=\omega \tau$ be the non-dimensional frequency, the corresponding boundary conditions are given by

$$
\begin{gathered}
\bar{P}(0, \bar{t})=\frac{i}{2} e^{-i \bar{\omega} \bar{t}}+\text { c.c. } \\
\bar{P}(\infty, \bar{t})=0
\end{gathered}
$$

Let $\bar{P}=\bar{P}_{0}+\varepsilon \bar{P}_{1}+\varepsilon^{2} \bar{P}_{2}$. Then Equation (2.3.2) becomes

$$
\begin{aligned}
\frac{\partial^{2} \bar{P}_{0}}{\partial \bar{t}^{2}}+\frac{\partial \bar{P}_{0}}{\partial \bar{t}}-\frac{\partial^{2} \bar{P}_{0}}{\partial \bar{x}^{2}} & +\varepsilon\left(\frac{\partial^{2} \bar{P}_{1}}{\partial \bar{t}^{2}}+\frac{\partial \bar{P}_{1}}{\partial \bar{t}}-\frac{\partial^{2} \bar{P}_{1}}{\partial \bar{x}^{2}}-\bar{P}_{0} \frac{\partial \bar{P}_{0}}{\partial \bar{t}}\right) \\
& +\varepsilon^{2}\left(\frac{\partial^{2} \bar{P}_{2}}{\partial \bar{t}^{2}}+\frac{\partial \bar{P}_{2}}{\partial \bar{t}}-\frac{\partial^{2} \bar{P}_{2}}{\partial \bar{x}^{2}}-\bar{P}_{0} \frac{\partial \bar{P}_{1}}{\partial \bar{t}}-\bar{P}_{1} \frac{\partial \bar{P}_{0}}{\partial \bar{t}}\right)+O\left(\varepsilon^{3}\right)=0
\end{aligned}
$$

To be zero, each power of $\varepsilon$ must vanish separately. Thus, we have the following system of coupled partial differential equations

$$
\begin{gathered}
\frac{\partial^{2} \bar{P}_{0}}{\partial \bar{t}^{2}}+\frac{\partial \bar{P}_{0}}{\partial \bar{t}}-\frac{\partial^{2} \bar{P}_{0}}{\partial \bar{x}^{2}}=0 \\
\frac{\partial^{2} \bar{P}_{1}}{\partial \bar{t}^{2}}+\frac{\partial \bar{P}_{1}}{\partial \bar{t}}-\frac{\partial^{2} \bar{P}_{1}}{\partial \bar{x}^{2}}=\bar{P}_{0} \frac{\partial \bar{P}_{0}}{\partial \bar{t}} \\
\frac{\partial^{2} \bar{P}_{2}}{\partial \bar{t}^{2}}+\frac{\partial \bar{P}_{2}}{\partial \bar{t}}-\frac{\partial^{2} \bar{P}_{2}}{\partial \bar{x}^{2}}=\bar{P}_{0} \frac{\partial \bar{P}_{1}}{\partial \bar{t}}+\bar{P}_{1} \frac{\partial \bar{P}_{0}}{\partial \bar{t}}
\end{gathered}
$$


For the sake of mathematical simplicity, we assume general solutions of the form

$$
\bar{P}_{n}(\bar{x}, \bar{t})=\frac{i}{2} \bar{V}_{n}(\bar{x}) e^{-i(n+1) \bar{\omega} \bar{t}}+\text { c.c. },
$$

where Equation (2.3.8) can be written as $\bar{P}_{n}=\overline{\mathcal{P}}_{n}+$ c.c. .

\subsection{The Zeroth-Order Term}

Substituting Equation (2.3.8) (with $n=0$ ) into Equation (2.3.5) gives

$$
\frac{\partial^{2}}{\partial \bar{t}^{2}}\left[\frac{i}{2} \bar{V}_{0}(\bar{x}) e^{-i \bar{\omega} \bar{t}}\right]+\frac{\partial}{\partial \bar{t}}\left[\frac{i}{2} \bar{V}_{0}(\bar{x}) e^{-i \bar{\omega} \bar{t}}\right]-\frac{\partial^{2}}{\partial \bar{x}^{2}}\left[\frac{i}{2} \bar{V}_{0}(\bar{x}) e^{-i \bar{\omega} \bar{t}}\right]=0,
$$

or

$$
\frac{\partial^{2}}{\partial \bar{t}^{2}}\left[\bar{V}_{0}(\bar{x}) e^{-i \bar{\omega} \bar{t}}\right]+\frac{\partial}{\partial \bar{t}}\left[\bar{V}_{0}(\bar{x}) e^{-i \bar{\omega} \bar{t}}\right]-\frac{\partial^{2}}{\partial \bar{x}^{2}}\left[\bar{V}_{0}(\bar{x}) e^{-i \bar{\omega} \bar{t}}\right]=0
$$

or

$$
-\bar{\omega}^{2} \bar{V}_{0}(\bar{x}) e^{-i \bar{\omega} \bar{t}}-i \bar{\omega} \bar{V}_{0}(\bar{x}) e^{-i \bar{\omega} \bar{t}}-\bar{V}_{0}^{\prime \prime}(\bar{x}) e^{-i \bar{\omega} \bar{t}}=0
$$

or, dividing through by $e^{-i \bar{\omega} \bar{t}}$,

$$
-\bar{\omega}^{2} \bar{V}_{0}(\bar{x})-i \bar{\omega} \bar{V}_{0}(\bar{x})-\bar{V}_{0}^{\prime \prime}(\bar{x})=0,
$$

or, letting $\bar{k}_{0}^{2}=\bar{\omega}^{2}+i \bar{\omega}$,

$$
\bar{V}_{0}^{\prime \prime}(\bar{x})+\bar{k}_{0}^{2} \bar{V}_{0}(\bar{x})=0 .
$$


The general solution of Equation (2.4.1) is given by

$$
\bar{V}_{0}(\bar{x})=\bar{A}_{0} e^{i \bar{k}_{0} \bar{x}}+\bar{B}_{0} e^{-i \bar{k}_{0} \bar{x}}
$$

Since $\bar{k}_{0}=\bar{\alpha}_{0}+i \bar{\beta}_{0} \quad\left(\bar{\alpha}_{0}, \bar{\beta}_{0}>0\right)$, we have $e^{-i \bar{k}_{0} \bar{x}}=e^{-i\left(\bar{\alpha}_{0}+i \bar{\beta}_{0}\right) \bar{x}}=e^{-i \bar{\alpha}_{0} \bar{x}} \cdot e^{\bar{\beta}_{0} \bar{x}} \rightarrow \infty$ as $\bar{x} \rightarrow \infty$, which implies $\bar{B}_{0}=0$ and

$$
\bar{V}_{0}(\bar{x})=\bar{A}_{0} e^{i \bar{k}_{0} \bar{x}}
$$

Then

$$
\overline{\mathcal{P}_{0}}(\bar{x}, \bar{t})=\frac{i}{2} \bar{A}_{0} e^{i \bar{k}_{0} \bar{x}} \cdot e^{-i \bar{\omega} \bar{t}}
$$

From the first $\mathrm{BC}$, we have

$$
\overline{\mathcal{P}_{0}}(0, \bar{t})=\frac{i}{2} \bar{A}_{0} e^{-i \bar{\omega} \bar{t}}=\frac{i}{2} e^{-i \bar{\omega} \bar{t}}
$$

Thus, $\bar{A}_{0}=1$, and so

$$
\overline{\mathcal{P}_{0}}(x, \bar{t})=\frac{i}{2} e^{i\left(\bar{k}_{0} \bar{x}-\bar{\omega} \bar{t}\right)} .
$$

Figure 1 shows the plot of $\left|\overline{\mathcal{P}_{0}}\right|$ versus position for several values of frequency. Here we see that $\left|\overline{\mathcal{P}_{0}}\right| \leq 1$ for all $\bar{x} \neq 0$. In particular, $\left|\overline{\mathcal{P}_{0}}\right|$ is monotonically decreasing over $\bar{x}$ with its maximum value of 1 at $\bar{x}=0$. 


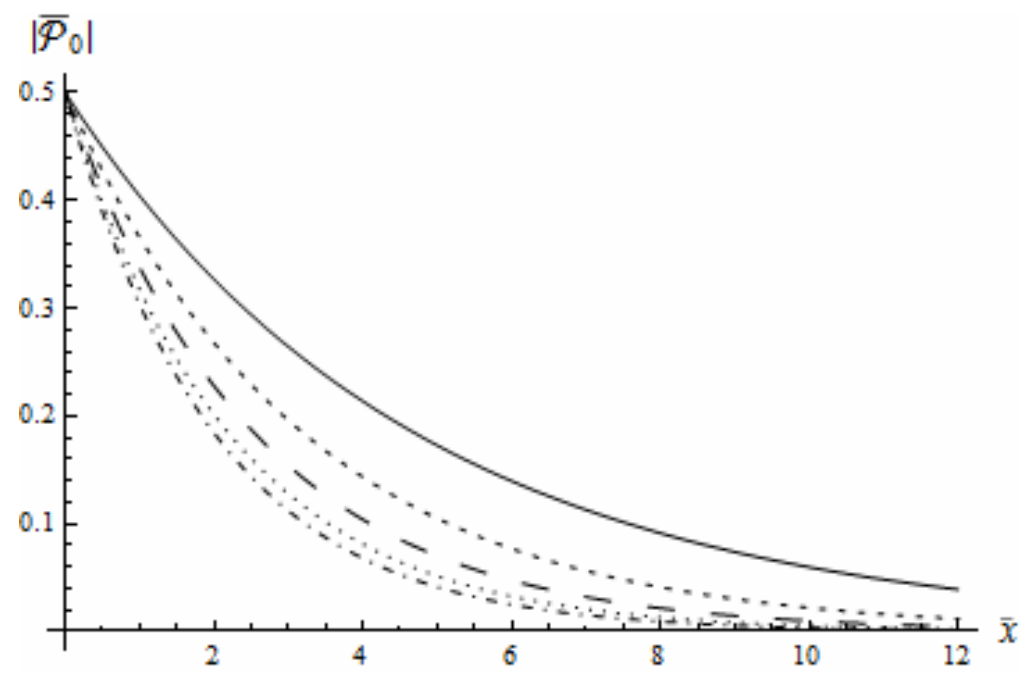

Figure 1. $\left|\overline{\mathcal{P}_{0}}\right|$ versus $\bar{x}$ for $\bar{\omega}=0.1$ (solid), 0.25 (short-dashed), 0.5 (dotted), 1.0 (dot-dashed) and 10.0 (long-dashed).

\subsection{The First-Order Correction}

Substituting Equation (2.3.8) (with $n=1$ ) into Equation (2.3.6) gives

$$
\begin{gathered}
\frac{\partial^{2}}{\partial \bar{t}^{2}}\left[\frac{i}{2} \bar{V}_{1}(\bar{x}) e^{-2 i \bar{\omega} \bar{t}}\right]+\frac{\partial}{\partial \bar{t}}\left[\frac{i}{2} \bar{V}_{1}(\bar{x}) e^{-2 i \bar{\omega} \bar{t}}\right]-\frac{\partial^{2}}{\partial \bar{x}^{2}}\left[\frac{i}{2} \bar{V}_{1}(\bar{x}) e^{-2 i \bar{\omega} \bar{t}}\right] \\
=\frac{i}{2} \bar{V}_{0}(\bar{x}) e^{-i \bar{\omega} \bar{t}} \cdot \frac{\partial}{\partial \bar{t}}\left[\frac{i}{2} \bar{V}_{0}(\bar{x}) e^{-i \bar{\omega} \bar{t}}\right],
\end{gathered}
$$

or

$$
\begin{gathered}
\frac{\partial^{2}}{\partial \bar{t}^{2}}\left[\bar{V}_{1}(\bar{x}) e^{-2 i \bar{\omega} \bar{t}}\right]+\frac{\partial}{\partial \bar{t}}\left[\bar{V}_{1}(\bar{x}) e^{-2 i \bar{\omega} \bar{t}}\right]-\frac{\partial^{2}}{\partial \bar{x}^{2}}\left[\bar{V}_{1}(\bar{x}) e^{-2 i \bar{\omega} \bar{t}}\right] \\
=\left(\frac{i}{2}\right)^{2} \bar{V}_{0}(\bar{x}) e^{-i \bar{\omega} \bar{t}} \cdot \frac{\partial}{\partial \bar{t}}\left[\bar{V}_{0}(\bar{x}) e^{-i \bar{\omega} \bar{t}}\right]
\end{gathered}
$$


or

$$
-4 \bar{\omega} \bar{V}_{1}(\bar{x}) e^{-2 i \bar{\omega} \bar{t}}-2 i \bar{\omega} \bar{V}_{1}(\bar{x}) e^{-2 i \bar{\omega} \bar{t}}-\bar{V}_{1}^{\prime \prime}(\bar{x}) e^{-2 i \bar{\omega} \bar{t}}=-i \bar{\omega} \bar{V}_{0}^{2}(\bar{x}) e^{-2 i \bar{\omega} \bar{t}}
$$

or, dividing through by $-e^{-2 i \bar{\omega} \bar{t}}$,

$$
4 \bar{\omega} \bar{V}_{1}(\bar{x})+2 i \bar{\omega} \bar{V}_{1}(\bar{x})+\bar{V}_{1}^{\prime \prime}(\bar{x})=i \bar{\omega} \bar{V}_{0}^{2}(\bar{x})
$$

or, letting $\bar{k}_{1}^{2}=4 \bar{\omega}^{2}+2 i \bar{\omega}$,

$$
\bar{V}_{1}^{\prime \prime}(\bar{x})+\bar{k}_{1}^{2} \bar{V}_{1}(\bar{x})=i \bar{\omega} \bar{V}_{0}^{2}(\bar{x}) .
$$

The general solution is given by

$$
\bar{V}_{1}(\bar{x})=\bar{A}_{1} e^{i \bar{k}_{1} \bar{x}}+\bar{B}_{1} e^{-i \bar{k}_{1} \bar{x}}+\bar{C}_{1} e^{2 i \bar{k}_{0} \bar{x}} .
$$

Again, we must have $\bar{B}_{1}=0$. Therefore,

$$
\bar{V}_{1}(\bar{x})=\bar{A}_{1} e^{i \bar{k}_{1} \bar{x}}+\bar{C}_{1} e^{2 i \bar{k}_{0} \bar{x}}
$$

Substituting Equation (2.5.2) into Equation (2.5.1) gives

$$
-\bar{k}_{1}^{2} \bar{A}_{1} e^{i \bar{k}_{1} \bar{x}}-4 \bar{k}_{0}^{2} \bar{C}_{1} e^{2 i \bar{k}_{0} \bar{x}}+\bar{k}_{1}^{2} \bar{A}_{1} e^{i \bar{k}_{1} \bar{x}}+\bar{k}_{1}^{2} \bar{C}_{1} e^{2 i \bar{k}_{0} \bar{x}}=-\frac{\bar{\omega}}{2} e^{2 i \bar{k}_{0} \bar{x}}
$$

or

$$
\left(\bar{k}_{1}^{2}-4 \bar{k}_{0}^{2}\right) \bar{C}_{1} e^{2 i \bar{k}_{0} \bar{x}}=-\frac{\bar{\omega}}{2} e^{2 i \bar{k}_{0} \bar{x}}
$$


Equating coefficients in Equation (2.5.3) gives

$$
\left(\bar{k}_{1}^{2}-4 \bar{k}_{0}^{2}\right) \bar{C}_{1}=-\frac{\bar{\omega}}{2}
$$

or, using the definitions of $\bar{k}_{0}^{2}$ and $\bar{k}_{1}^{2}$, we have $\bar{k}_{1}^{2}-4 \bar{k}_{0}^{2}=-2 i \bar{\omega}$. Then

$$
-2 i \bar{\omega} \bar{C}_{1}=-\frac{\bar{\omega}}{2}
$$

which gives $\bar{C}_{1}=\frac{1}{4 i}=-\frac{i}{4}$, and so

$$
\bar{V}_{1}(\bar{x})=\bar{A}_{1} e^{i \bar{k}_{1} \bar{x}}-\frac{i}{4} e^{2 i \bar{k}_{0} \bar{x}}
$$

or

$$
\overline{\mathcal{P}_{1}}(\bar{x}, \bar{t})=\frac{i}{2}\left(\bar{A}_{1} e^{i \bar{k}_{1} \bar{x}}-\frac{i}{4} e^{2 i \bar{k}_{0} \bar{x}}\right) e^{-2 i \bar{\omega} \bar{t}}
$$

From the first BC, we have

$$
\overline{\mathcal{P}_{1}}(0, \bar{t})=\frac{i}{2}\left(\overline{A_{1}}-\frac{i}{4}\right) e^{-2 i \bar{\omega} \bar{t}}=0 .
$$

Equating coefficients in Equation (2.5.4) gives

$$
\bar{A}_{1}-\frac{i}{4}=0,
$$

Thus, $\bar{A}_{1}=\frac{i}{4}$, and so

$$
\overline{\mathcal{P}_{1}}(\bar{x}, \bar{t})=\left(\frac{i}{2}\right)^{2} \cdot \frac{1}{2}\left[e^{i\left(\bar{k}_{1} \bar{x}-2 \bar{\omega} \bar{t}\right)}-e^{2 i\left(\bar{k}_{0} \bar{x}-2 i \bar{\omega} \bar{t}\right)}\right]
$$


Figure 2 shows the plot of $\left|\overline{\mathcal{P}_{1}}\right|$ versus position for several values of frequency. Here we see that $\left|\overline{\mathcal{P}_{1}}\right|$ is strictly less than 1 for all $\bar{x}$, achieves a unique maximum and decays to zero as $\bar{x} \rightarrow \infty$. Furthermore, the location of the peak of $\left|\overline{\mathcal{P}}_{1}\right|, \bar{x}_{\max }$, shifts to the left as the frequency increases and to the right as frequency decreases. In Chapter 5, we investigate the relationship of $\bar{x}_{\max }$ to frequency. Particular attention is paid to the limits of low and high frequencies and approximations to $\bar{x}_{\max }$ are developed in these regions.

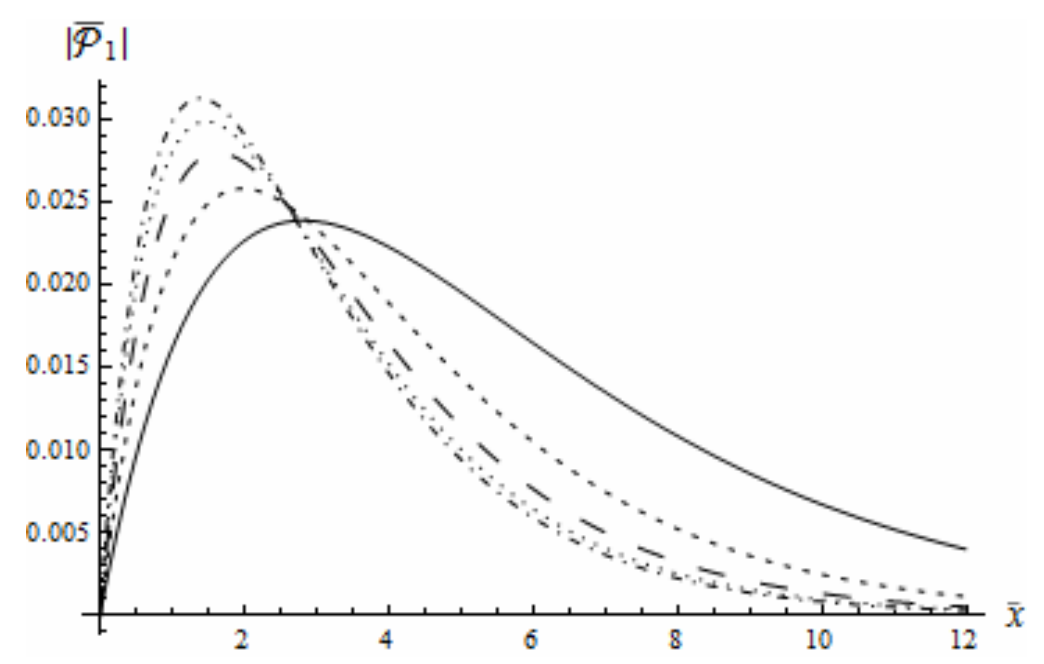

Figure 2. $\left|\overline{\mathcal{P}_{1}}\right|$ versus $\bar{x}$ for $\bar{\omega}=\mathbf{0 . 1}$ (solid), 0.25 (short-dashed), 0.5 (dotted), 1.0 (dot-dashed) and 10.0 (long-dashed). 


\subsection{The Second-Order Correction}

Substituting Equation (2.3.8) (with $n=2$ ) into Equation (2.3.7) gives

$$
\begin{aligned}
& \frac{\partial^{2}}{\partial \bar{t}^{2}}\left[\frac{i}{2} \bar{V}_{2}(\bar{x}) e^{-3 i \bar{\omega} \bar{t}}\right]+\frac{\partial}{\partial \bar{t}}\left[\frac{i}{2} \bar{V}_{2}(\bar{x}) e^{-3 i \bar{\omega} \bar{t}}\right]-\frac{\partial^{2}}{\partial \bar{x}^{2}}\left[\frac{i}{2} \bar{V}_{2}(\bar{x}) e^{-3 i \bar{\omega} \bar{t}}\right] \\
& =\frac{i}{2} \bar{V}_{0}(\bar{x}) e^{-i \bar{\omega} \bar{t}} \cdot \frac{\partial}{\partial \bar{t}}\left[\frac{i}{2} \bar{V}_{1}(\bar{x}) e^{-2 i \bar{\omega} \bar{t}}\right]+\frac{i}{2} \bar{V}_{1}(\bar{x}) e^{-2 i \bar{\omega} \bar{t}} \cdot \frac{\partial}{\partial \bar{t}}\left[\frac{i}{2} \bar{V}_{0}(\bar{x}) e^{-i \bar{\omega} \bar{t}}\right]
\end{aligned}
$$

or

$$
\begin{aligned}
& \frac{i}{2} \frac{\partial^{2}}{\partial \bar{t}^{2}}\left[\bar{V}_{2}(\bar{x}) e^{-3 i \bar{\omega} \bar{t}}\right]+\frac{i}{2} \frac{\partial}{\partial \bar{t}}\left[\bar{V}_{2}(\bar{x}) e^{-3 i \bar{\omega} \bar{t}}\right]-\frac{i}{2} \frac{\partial^{2}}{\partial \bar{x}^{2}}\left[\bar{V}_{2}(\bar{x}) e^{-3 i \bar{\omega} \bar{t}}\right] \\
& =\left(\frac{i}{2}\right)^{2} \bar{V}_{0}(\bar{x}) e^{-i \bar{\omega} \bar{t}} \cdot \frac{\partial}{\partial \bar{t}}\left[\bar{V}_{1}(\bar{x}) e^{-2 i \bar{\omega} \bar{t}}\right]+\left(\frac{i}{2}\right)^{2} \bar{V}_{1}(\bar{x}) e^{-2 i \bar{\omega} \bar{t}} \cdot \frac{\partial}{\partial \bar{t}}\left[\bar{V}_{0}(\bar{x}) e^{-i \bar{\omega} \bar{t}}\right]
\end{aligned}
$$

or

$$
-\left(9 \bar{\omega}^{2}+3 i \bar{\omega}\right) \bar{V}_{2} e^{-3 i \bar{\omega} \bar{t}}-\bar{V}_{2}^{\prime \prime} e^{-3 i \bar{\omega} \bar{t}}=-\frac{i}{2}(3 i \bar{\omega}) \bar{V}_{0} \cdot \bar{V}_{1}
$$

or, letting $\bar{k}_{2}^{2}=9 \bar{\omega}^{2}+3 i \bar{\omega}$,

$$
\bar{V}_{2}^{\prime \prime}+\bar{k}_{2}^{2} \bar{V}_{2}=-\frac{3 \bar{\omega}}{2} \bar{V}_{0} \cdot \bar{V}_{1}
$$

Now, $\bar{V}_{0}(\bar{x}) \cdot \bar{V}_{1}(\bar{x})=\frac{i}{4} e^{i \bar{k}_{0} \bar{x}}\left(e^{i \bar{k}_{1} \bar{x}}-e^{2 i \bar{k}_{0} \bar{x}}\right)=\frac{i}{4}\left[e^{i\left(\bar{k}_{0}+\bar{k}_{1}\right) \bar{x}}-e^{3 i \bar{k}_{0} \bar{x}}\right]$.

Thus

$$
-\frac{3 \bar{\omega}}{2} \bar{V}_{0}(\bar{x}) \cdot \bar{V}_{1}(\bar{x})=-\frac{3 \bar{\omega}}{2} \cdot \frac{i}{4} e^{i \bar{k}_{0} \bar{x}}\left(e^{i \bar{k}_{1} \bar{x}}-e^{2 i \bar{k}_{0} \bar{x}}\right)=-\frac{3 i \bar{\omega}}{8}\left[e^{i\left(\bar{k}_{0}+\bar{k}_{1}\right) \bar{x}}-e^{3 i \bar{k}_{0} \bar{x}}\right]
$$


Then, Equation (2.6.1) becomes

$$
\bar{V}_{2}^{\prime \prime}+\bar{k}_{2}^{2} \bar{V}_{2}=-\frac{3 i \bar{\omega}}{8}\left[e^{i\left(\bar{k}_{0}+\bar{k}_{1}\right) \bar{x}}-e^{3 i \bar{k}_{0} \bar{x}}\right]
$$

The general solution of Equation (2.6.2) is

$$
\bar{V}_{2}(\bar{x})=\bar{A}_{2} e^{i \bar{k}_{2} \bar{x}}+\bar{B}_{2} e^{-i \bar{k}_{2} \bar{x}}+\bar{C}_{2} e^{i\left(\bar{k}_{0}+\bar{k}_{1}\right) \bar{x}}+\bar{D}_{2} e^{3 i \bar{k}_{0} \bar{x}}
$$

Once again, we must have $\bar{B}_{2}=0$. Then

$$
\bar{V}_{2}(\bar{x})=\bar{A}_{2} e^{i \bar{k}_{2} \bar{x}}+\bar{C}_{2} e^{i\left(\bar{k}_{0}+\bar{k}_{1}\right) \bar{x}}+\bar{D}_{2} e^{3 i \bar{k}_{0} \bar{x}}
$$

Differentiating Equation (2.6.3) twice with respect to $\bar{x}$ gives

$$
\bar{V}_{2}^{\prime \prime}(\bar{x})=-\bar{k}_{2}^{2} \bar{A}_{2} e^{i \bar{k}_{2} \bar{x}}-\left(\bar{k}_{0}+\bar{k}_{1}\right)^{2} \bar{C}_{2} e^{i\left(\bar{k}_{0}+\bar{k}_{1}\right) \bar{x}}-9 \bar{k}_{0}^{2} \bar{D}_{2} e^{3 i \bar{k}_{0} \bar{x}}
$$

\section{Moreover}

$$
\bar{k}_{2}^{2} \bar{V}_{2}(\bar{x})=\bar{k}_{2}^{2} \bar{A}_{2} e^{i \bar{k}_{2} \bar{x}}+\bar{k}_{2}^{2} \bar{C}_{2} e^{i\left(\bar{k}_{0}+\bar{k}_{1}\right) \bar{x}}+\bar{k}_{2}^{2} \bar{D}_{2} e^{3 i \bar{k}_{0} \bar{x}}
$$

Therefore

$$
\bar{V}_{2}^{\prime \prime}+\bar{k}_{2}^{2} \bar{V}_{2}=\left[\bar{k}_{2}^{2}-\left(\bar{k}_{0}+\bar{k}_{1}\right)^{2}\right] \bar{C}_{2} e^{i\left(\bar{k}_{0}+\bar{k}_{1}\right) \bar{x}}+\left(\bar{k}_{2}^{2}-9 \bar{k}_{0}^{2}\right) \bar{D}_{2} e^{3 i \bar{k}_{0} \bar{x}} .
$$

Combining Equation (2.6.2) and Equation (2.6.4) gives

$$
\begin{gathered}
{\left[\bar{k}_{2}^{2}-\left(\bar{k}_{0}+\bar{k}_{1}\right)^{2}\right] \bar{C}_{2} e^{i\left(\bar{k}_{0}+\bar{k}_{1}\right) \bar{x}}+\left(\bar{k}_{2}^{2}-9 \bar{k}_{0}^{2}\right) \bar{D}_{2} e^{3 i \bar{k}_{0} \bar{x}}} \\
=-\frac{3 i \bar{\omega}}{8} e^{i\left(\bar{k}_{0}+\bar{k}_{1}\right) \bar{x}}+\frac{3 i \bar{\omega}}{8} e^{3 i \bar{k}_{0} \bar{x}}
\end{gathered}
$$


Then, equating exponentials in Equation (2.6.5), we have

$$
\begin{gathered}
{\left[\bar{k}_{2}^{2}-\left(\bar{k}_{0}+\bar{k}_{1}\right)^{2}\right] \bar{C}_{2}=-\frac{3 i \bar{\omega}}{8}} \\
\left(\bar{k}_{2}^{2}-9 \bar{k}_{0}^{2}\right) \bar{D}_{2}=\frac{3 i \bar{\omega}}{8},
\end{gathered}
$$

or, since $\bar{k}_{2}^{2}-9 \bar{k}_{0}^{2}=9 \bar{\omega}^{2}+3 i \bar{\omega}-9\left(\bar{\omega}^{2}+i \bar{\omega}\right)=-6 i \bar{\omega}$ and

$$
\begin{aligned}
\bar{k}_{2}^{2}-\left(\bar{k}_{0}+\bar{k}_{1}\right)^{2} & =\bar{k}_{2}^{2}-\left(\bar{k}_{0}^{2}+\bar{k}_{1}^{2}+2 \bar{k}_{0} \cdot \bar{k}_{1}\right) \\
& =9 \bar{\omega}^{2}+3 i \bar{\omega}-\left(\bar{\omega}^{2}+i \bar{\omega}+4 \bar{\omega}^{2}+2 i \bar{\omega}+2 \bar{k}_{0} \cdot \bar{k}_{1}\right) \\
& =9 \bar{\omega}^{2}+3 i \bar{\omega}-\left(5 \bar{\omega}^{2}+3 i \bar{\omega}+2 \bar{k}_{0} \cdot \bar{k}_{1}\right) \\
& =4 \bar{\omega}^{2}-2 \bar{k}_{0} \cdot \bar{k}_{1}
\end{aligned}
$$

Equations (2.6.6) become

$$
\begin{gathered}
2\left(2 \bar{\omega}^{2}-\bar{k}_{0} \cdot \bar{k}_{1}\right) \bar{C}_{2}=-\frac{3 i \bar{\omega}}{8} \\
-6 i \bar{\omega} \bar{D}_{2}=\frac{3 i \bar{\omega}}{8}
\end{gathered} .
$$

Solving (2.6.7) for $\bar{C}_{2}$ and $\bar{D}_{2}$ gives

$$
\begin{gathered}
\bar{C}_{2}=-\frac{3 i \bar{\omega}}{16\left(2 \bar{\omega}^{2}-\bar{k}_{0} \cdot \bar{k}_{1}\right)} . \\
\bar{D}_{2}=-\frac{1}{16}
\end{gathered}
$$


Then, Equation (2.6.3) becomes

$$
\bar{V}_{2}(\bar{x})=\bar{A}_{2} e^{i \bar{k}_{2} \bar{x}}-\frac{3 i \bar{\omega}}{16\left(2 \bar{\omega}^{2}-\bar{k}_{0} \cdot \bar{k}_{1}\right)} e^{i\left(\bar{k}_{0}+\bar{k}_{1}\right) \bar{x}}-\frac{1}{16} e^{3 i \bar{k}_{0} \bar{x}}
$$

and so

$$
\overline{\mathcal{P}_{2}}(\bar{x}, \bar{t})=\frac{i}{2}\left[\bar{A}_{2} e^{i \bar{k}_{2} \bar{x}}-\frac{3 i \bar{\omega}}{16\left(2 \bar{\omega}^{2}-\bar{k}_{0} \cdot \bar{k}_{1}\right)} e^{i\left(\bar{k}_{0}+\bar{k}_{1}\right) \bar{x}}-\frac{1}{16} e^{3 i \bar{k}_{0} \bar{x}}\right] e^{-3 \bar{\omega} \bar{t}}
$$

From the first $\mathrm{BC}$, we have

$$
\overline{\mathcal{P}_{2}}(0, \bar{t})=\frac{i}{2}\left[\bar{A}_{2}-\frac{3 i \bar{\omega}}{16\left(2 \bar{\omega}^{2}-\bar{k}_{0} \cdot \bar{k}_{1}\right)}-\frac{1}{16}\right] e^{-3 \bar{\omega} \bar{t}}=0 .
$$

Then

$$
\bar{A}_{2}-\frac{3 i \bar{\omega}}{16\left(2 \bar{\omega}^{2}-\bar{k}_{0} \cdot \bar{k}_{1}\right)}-\frac{1}{16}=0
$$

or

$$
\bar{A}_{2}=\frac{1}{16}+\frac{3 i \bar{\omega}}{16\left(2 \bar{\omega}^{2}-\bar{k}_{0} \cdot \bar{k}_{1}\right)}=\frac{2 \bar{\omega}^{2}-\bar{k}_{0} \cdot \bar{k}_{1}+3 i \bar{\omega}}{16\left(2 \bar{\omega}^{2}-\bar{k}_{0} \cdot \bar{k}_{1}\right)}
$$

Thus

$$
\overline{\mathcal{P}_{2}}(\bar{x}, \bar{t})=\frac{i}{2}\left[\frac{2 \bar{\omega}^{2}-\bar{k}_{0} \cdot \bar{k}_{1}+3 i \bar{\omega}}{16\left(2 \bar{\omega}^{2}-\bar{k}_{0} \cdot \bar{k}_{1}\right)} e^{i \bar{k}_{2} \bar{x}}-\frac{3 i \bar{\omega}}{16\left(2 \bar{\omega}^{2}-\bar{k}_{0} \cdot \bar{k}_{1}\right)} e^{i\left(\bar{k}_{0}+\bar{k}_{1}\right) \bar{x}}-\frac{1}{16} e^{3 i \bar{k}_{0} \bar{x}}\right] e^{-3 \bar{\omega} \bar{t}}
$$


or

$\overline{\mathcal{P}_{2}}(\bar{x}, \bar{t})=-\frac{i}{2} \cdot \frac{e^{-3 \bar{\omega} \bar{t}}}{4}\left[\frac{2 \bar{\omega}^{2}-\bar{k}_{0} \cdot \bar{k}_{1}+3 i \bar{\omega}}{4\left(\bar{k}_{0} \cdot \bar{k}_{1}-2 \bar{\omega}^{2}\right)} e^{i \bar{k}_{2} \bar{x}}+\frac{3 i \bar{\omega}}{4\left(2 \bar{\omega}^{2}-\bar{k}_{0} \cdot \bar{k}_{1}\right)} e^{i\left(\bar{k}_{0}+\bar{k}_{1}\right) \bar{x}}+\frac{1}{4} e^{3 i \bar{k}_{0} \bar{x}}\right]$,

or

$$
\overline{\mathcal{P}_{2}}(\bar{x}, \bar{t})=\left(\frac{i}{2}\right)^{3} \cdot \frac{1}{4}\left\{\bar{A}_{2}^{\prime} e^{i\left(\bar{k}_{2} \bar{x}-3 \bar{\omega} \bar{t}\right)}+\bar{B}_{2}^{\prime} e^{i\left[\left(\bar{k}_{0}+\bar{k}_{1}\right) \bar{x}-3 \bar{\omega} \bar{t}\right]}+e^{3 i\left(\bar{k}_{0} \bar{x}-\bar{\omega} \bar{t}\right)}\right\}
$$

where

$$
\bar{A}_{2}^{\prime}=\frac{2 \bar{\omega}^{2}-\bar{k}_{0} \cdot \bar{k}_{1}+3 i \bar{\omega}}{\bar{k}_{0} \cdot \bar{k}_{1}-2 \bar{\omega}^{2}}
$$

and

$$
\bar{B}_{2}^{\prime}=\frac{3 i \bar{\omega}}{\bar{k}_{0} \cdot \bar{k}_{1}-2 \bar{\omega}^{2}}
$$

Figure 3 shows the plot of $\left|\overline{\mathcal{P}_{2}}\right|$ versus position for several values of frequency. As with the plot of $\left|\overline{\mathcal{P}_{1}}\right|$, we see that $\left|\overline{\mathcal{P}_{2}}\right|$ is strictly less than 1 for all $\bar{x}$, achieves a unique maximum and decays to zero as $\bar{x} \rightarrow \infty$. Furthermore, the location of the peak of $\left|\overline{\mathcal{P}_{2}}\right|$, behaves as it did for $\left|\overline{\mathcal{P}_{1}}\right|$ frequency increases and to the right as frequency decreases. 


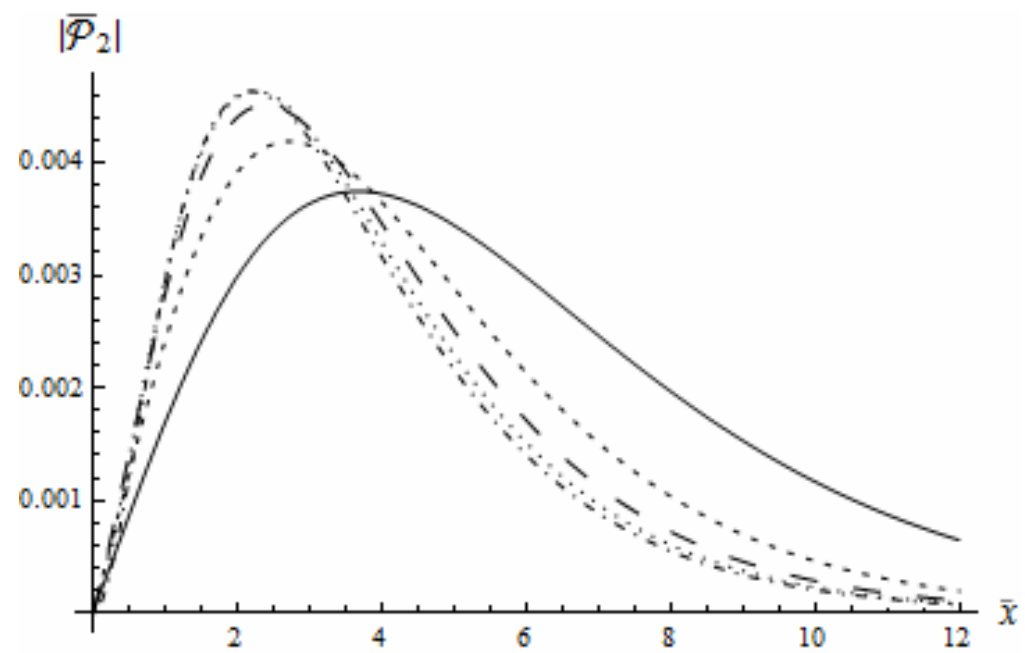

Figure 3. $\left|\overline{\mathcal{P}_{2}}\right|$ versus $\bar{x}$ for $\bar{\omega}=\mathbf{0 . 1}$ (solid), 0.25 (short-dashed), 0.5 (dotted), 1.0 (dot-dashed) and 10.0 (long-dashed). 


\section{Chapter 3. Dispersion Relations and Phase Speeds}

\subsection{Overview}

In this chapter, we derive expressions for the dispersion relations $\bar{k}_{n}(\bar{\omega})$ and the phase speeds $\bar{v}_{\mathrm{ph}, n}(\bar{\omega})$. Since $\bar{k}_{n} \in \mathbb{C}$, we must first determine the real part $\bar{\alpha}_{n}$ and imaginary part $\bar{\beta}_{n}$ of $\bar{k}_{n}$. The limits of low and high frequencies are also investigated for both $\bar{k}_{n}$ and $\bar{v}_{\mathrm{ph}, n}$.

\subsection{Dispersion Relations}

\section{Real and Imaginary Parts}

By inspection, we see that, for $n=0,1,2$,

$$
\bar{k}_{n}^{2}(\bar{\omega})=(n+1)^{2} \bar{\omega}^{2}+(n+1) i \bar{\omega} .
$$

We now express $\bar{k}_{n}$ in standard form. Let $\bar{\alpha}_{n}>0$ and $\bar{\beta}_{n}>0$ be the real and imaginary parts of $\bar{k}_{n}$, respectively. Then

$$
(n+1)^{2} \bar{\omega}^{2}+(n+1) i \bar{\omega}=\left(\bar{\alpha}_{n}+i \bar{\beta}_{n}\right)^{2}=\bar{\alpha}_{n}^{2}-\bar{\beta}_{n}^{2}+2 i \bar{\alpha}_{n} \bar{\beta}_{n}
$$

Equating real and imaginary parts gives the $2 \times 2$ system of equations

$$
\begin{aligned}
& (n+1)^{2} \bar{\omega}^{2}=\bar{\alpha}_{n}^{2}-\bar{\beta}_{n}^{2} \\
& (n+1) \bar{\omega}=2 \bar{\alpha}_{n} \bar{\beta}_{n}
\end{aligned}
$$


The second equation in (3.2.2) gives

$$
\bar{\beta}_{n}=\frac{(n+1) \bar{\omega}}{2 \bar{\alpha}_{n}}
$$

Substituting Equation (3.2.3) into the first equation in (3.2.2) gives

$$
(n+1)^{2} \bar{\omega}^{2}=\bar{\alpha}_{n}^{2}-\frac{(n+1)^{2} \bar{\omega}^{2}}{4 \bar{\alpha}_{n}^{2}}
$$

or

$$
4 \bar{\alpha}_{n}^{4}-4(n+1)^{2} \bar{\omega}^{2} \bar{\alpha}_{n}{ }^{2}-(n+1)^{2} \bar{\omega}^{2}=0,
$$

which is quadratic in $\bar{\alpha}_{n}{ }^{2}$. From the Quadratic Formula, we have

$$
\bar{\alpha}_{n}{ }^{2}=\frac{4(n+1)^{2} \bar{\omega}^{2}+\sqrt{16(n+1)^{4} \bar{\omega}^{4}+16(n+1)^{2} \bar{\omega}^{2}}}{8}
$$

where the positive root is taken since $\bar{\alpha}_{n} \in \mathbb{R}$, or

$$
\bar{\alpha}_{n}^{2}=\frac{(n+1)^{2} \bar{\omega}^{2}+\sqrt{(n+1)^{4} \bar{\omega}^{4}+(n+1)^{2} \bar{\omega}^{2}}}{2} .
$$

Thus, the real parts of the dispersion relations are given by

$$
\bar{\alpha}_{n}=\sqrt{\frac{(n+1)^{2} \bar{\omega}^{2}+\sqrt{(n+1)^{4} \bar{\omega}^{4}+(n+1)^{2} \bar{\omega}^{2}}}{2}},
$$

where the positive root is selected. Then, factoring out $\sqrt{(n+1) \bar{\omega} / 2}$ gives

$$
\bar{\alpha}_{n}(\bar{\omega})=\sqrt{\frac{(n+1) \bar{\omega}}{2}} \cdot \sqrt{\sqrt{(n+1)^{2} \bar{\omega}^{2}+1}+(n+1) \bar{\omega}} .
$$


From Equation (3.2.3), $\bar{\beta}_{n}$ is then given by

$$
\bar{\beta}_{n}=\frac{\frac{(n+1) \bar{\omega}}{2}}{\sqrt{\frac{(n+1) \bar{\omega}}{2}}} \cdot \frac{1}{\sqrt{\sqrt{(n+1)^{2} \bar{\omega}^{2}+1}+(n+1) \bar{\omega}}},
$$

or

$$
\bar{\beta}_{n}(\bar{\omega})=\sqrt{\frac{(n+1) \bar{\omega}}{2}} \cdot \sqrt{\sqrt{(n+1)^{2} \bar{\omega}^{2}+1}-(n+1) \bar{\omega}} .
$$

The (a) real part and (b) imaginary part of the dispersion relation are shown plotted against frequency for several modes in Figure 4. Here, we see that $\bar{\alpha}_{n}$ increases with increasing frequency. For large values of $\bar{\omega}, \bar{\alpha}_{n}$ appears to vary in, roughly, direct proportion with frequency. Moreover, this proportionality factor increases as $n$ increases. However, the behavior of $\bar{\beta}_{n}$ is quite different. We see that, for low frequencies, $\bar{\beta}_{n}$ increases with frequency, approaching the constant $1 / 2$. Note that as $n$ increases, $\bar{\beta}_{n}$ approaches this upper bound more rapidly. From [9], we see that for small frequencies, the medium assumes a diffusive-like nature. However, for large frequencies, the medium behaves similar to one exhibiting relaxation [27]. It is in this region we are most concerned, since combustion can only be sustained if energy propagates through the system. For mid-range frequencies, the behavior can be either diffusive or propagative. 

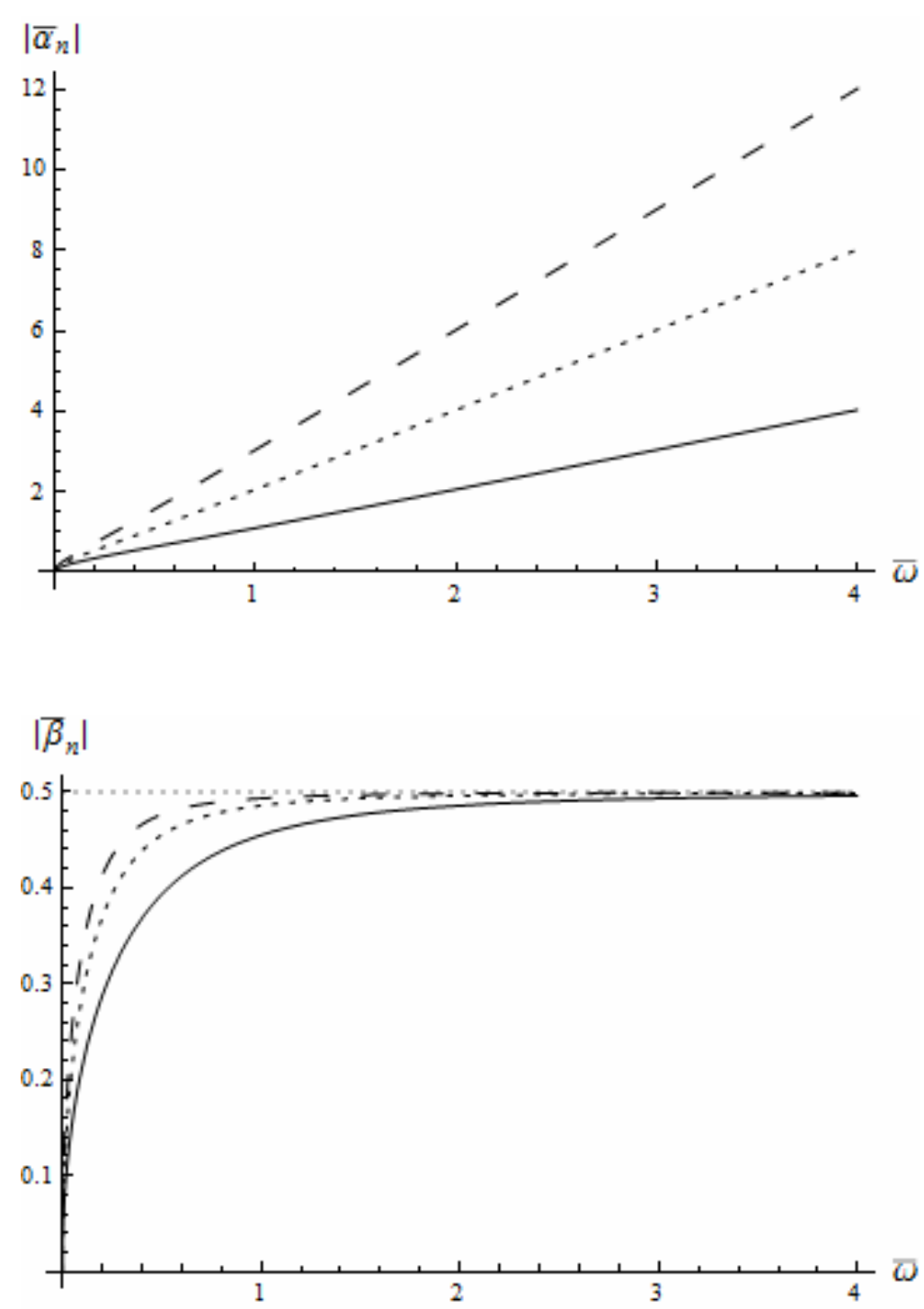

Figure 4 The (a) real part, $\bar{\alpha}_{n}$, and (b) imaginary part $\bar{\alpha}_{n}$, of the dispersion relations plotted versus frequency for $n=0$ (solid), $n=1$ (dotted) and $n=2$ (dashed). 


\section{Low-Frequency Regime}

As $\bar{\omega} \rightarrow 0, \sqrt{(n+1)^{2} \bar{\omega}^{2}+1}+(n+1) \bar{\omega} \rightarrow 1$. Thus, for $\bar{\omega} \ll 1$,

$$
\bar{\alpha}_{n}(\bar{\omega}) \approx \sqrt{\frac{(n+1) \bar{\omega}}{2}}
$$

Similarly, as $\bar{\omega} \rightarrow 0, \sqrt{(n+1)^{2} \bar{\omega}^{2}+1}-(n+1) \bar{\omega} \rightarrow 1$. Thus, for $\bar{\omega} \ll 1$,

$$
\bar{\beta}_{n}(\bar{\omega}) \approx \sqrt{\frac{(n+1) \bar{\omega}}{2}} .
$$

Thus, for small frequencies, $\bar{\alpha}_{n}$ and $\bar{\beta}_{n}$ are approximately proportional to $\sqrt{\bar{\omega}}$, indicating diffusive effects [8]. That is, the energy due to the harmonic boundary condition is not enough for propagating pressure waves to develop. Figure 5 shows plots of (a) $\bar{\alpha}_{n}$ and (b) $\bar{\beta}_{n}$ versus $\bar{\omega}$ for several modes in the low-frequency regime compared with the low-frquency limits given by Equation. Here, the $\sqrt{\bar{\omega}}$-type behavior of $\bar{\alpha}_{n}$ and $\bar{\beta}_{n}$ is readily seen. 


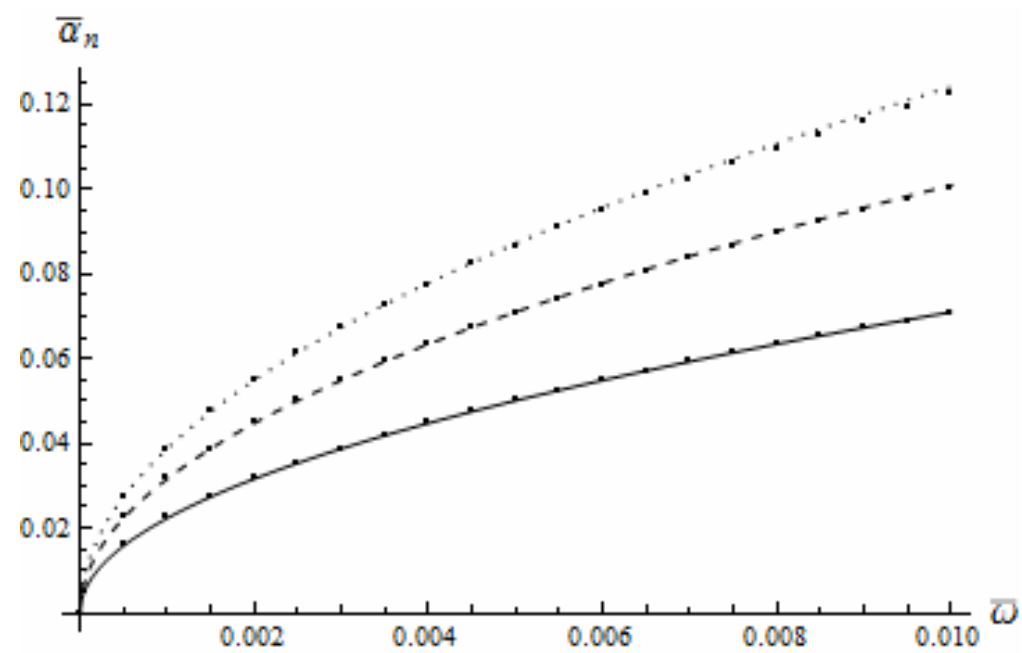

(a)

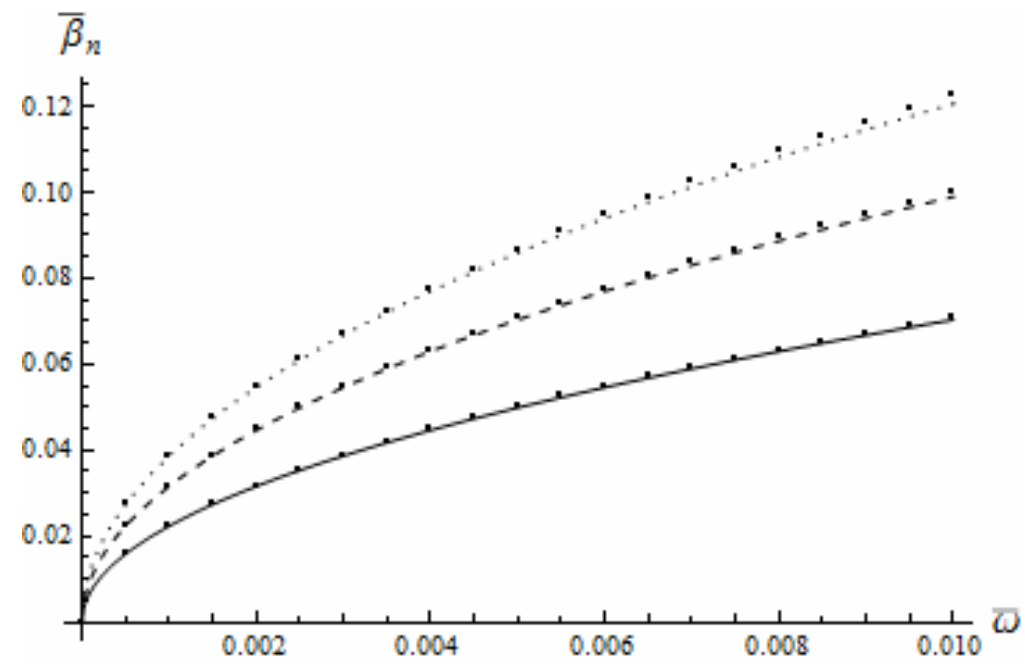

(b)

Figure 5. The (a) real part, $\bar{\alpha}_{n}$, and (b) imaginary part $\bar{\alpha}_{n}$, of the dispersion relations plotted versus frequency in the low-frequency region for $n=0$ (solid), $n=1$ (dashed) and $n=2$ (small dotted). Dots correspond to the analytic solution given by Equations (3.2.8) and (3.2.9). 


\section{High-Frequency regime}

Consider the following identity

$$
\sqrt{\sqrt{(n+1)^{2} \bar{\omega}^{2}+1}+(n+1) \bar{\omega}}=\sqrt{(n+1) \bar{\omega}} \cdot \sqrt{\sqrt{1+\frac{1}{(n+1)^{2} \bar{\omega}^{2}}}+1} .
$$

Thus, for fixed $n$, we have, for large $\bar{\omega}$, using the binomial approximation,

$$
\bar{\alpha}_{n}(\bar{\omega}) \approx(n+1) \bar{\omega} \cdot\left[1+\frac{1}{8(n+1)^{2} \bar{\omega}^{2}}\right]
$$

Similarly, for large $\bar{\omega}$,

$$
\bar{\beta}_{n}(\bar{\omega}) \approx \frac{1}{2} \cdot\left[1-\frac{1}{8(n+1)^{2} \bar{\omega}^{2}}\right] .
$$

Thus, for large $\bar{\omega}, \bar{\alpha}_{n}$ is in direct proportion to the frequency, indicating propagative effects. That is, the energy due to the harmonic boundary condition is large enough for pressure waves to propagate through the gas. $\bar{\beta}_{n}$, on the other hand, approaches the constant value of $\frac{1}{2}$ from below as $\bar{\omega}$ increases without bound. Figure 6 shows plots of (a) $\bar{\alpha}_{n}$ and (b) in the highfrequency region. Here, the linear behavior of frequency is verified for $\bar{\alpha}_{n}$ and $\bar{\beta}_{n} \approx 1 / 2$. 


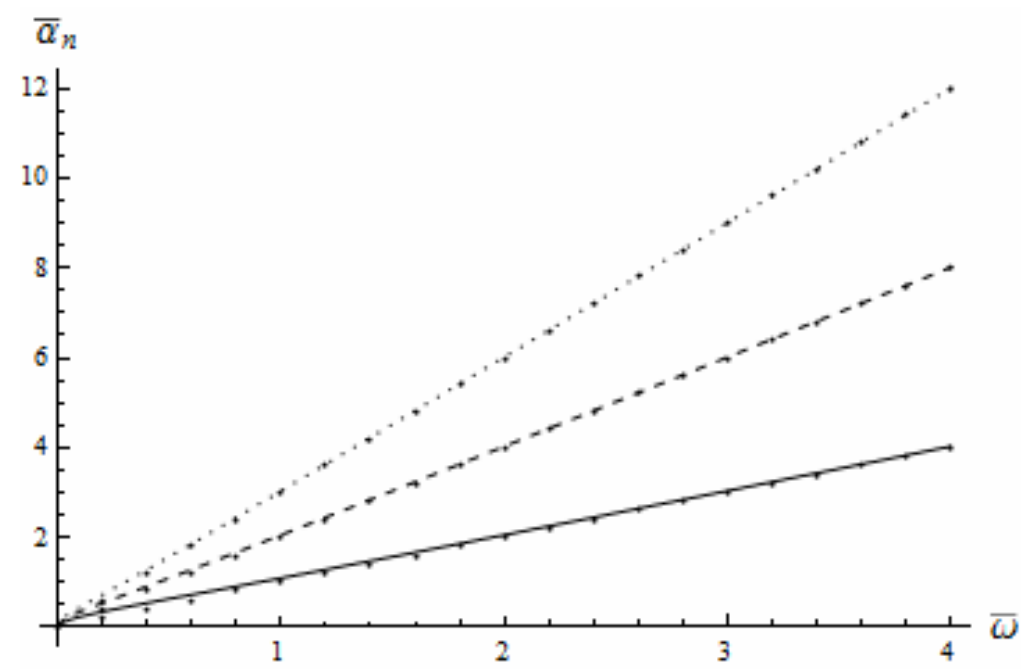

(a)

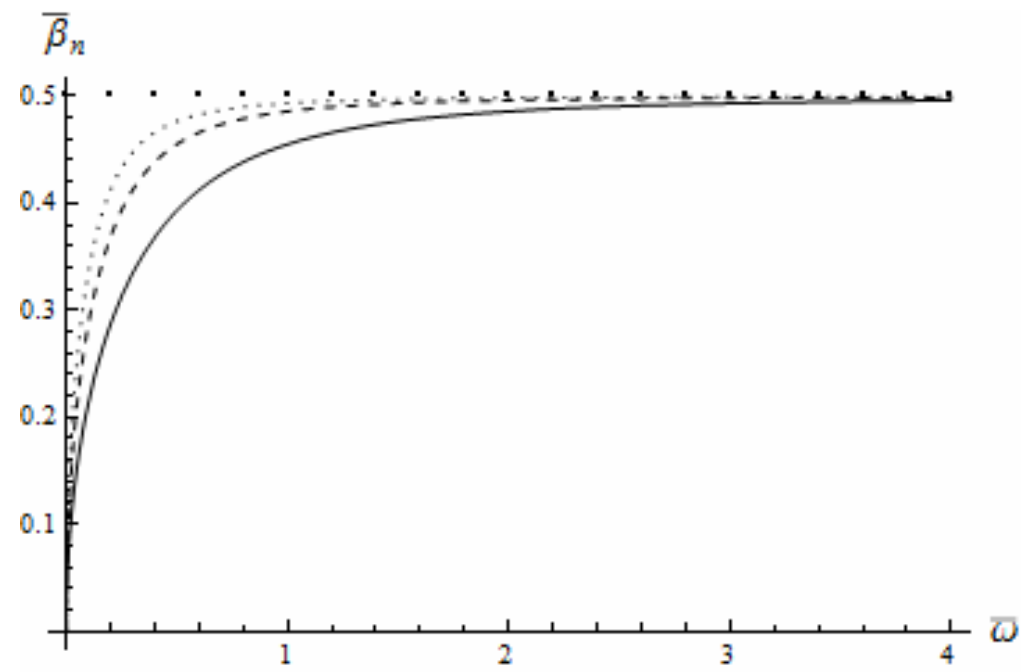

(b)

Figure 6. The (a) real part, $\bar{\alpha}_{n}$, and (b) imaginary part $\bar{\beta}_{n}$, of the dispersion relations plotted versus frequency in the high-frequency region for $n=0$ (solid), $n=1$ (dashed) and $n=2$ (small dotted). Dots correspond to the analytic solution given by Equations (3.2.10) and (3.2.11). 


\subsection{Phase Speeds}

The (non-dimensional) phase speed of the pressure wave is defined as

$$
\bar{v}_{\mathrm{ph}, n}(\bar{\omega})=\frac{\bar{\omega}}{\left|\bar{k}_{n}(\bar{\omega})\right|},
$$

where the modulus of $\bar{k}_{n}$ has been used since $\bar{k}_{n} \in \mathbb{C}$. We have $\left|\bar{k}_{n}\right|=\sqrt{\bar{\alpha}_{n}{ }^{2}+\bar{\beta}_{n}{ }^{2}}$. Now,

$$
\begin{aligned}
& \bar{\alpha}_{n}{ }^{2}=\frac{(n+1) \bar{\omega}}{2} \cdot\left[\sqrt{(n+1)^{2} \bar{\omega}^{2}+1}+(n+1) \bar{\omega}\right] \\
& \bar{\beta}_{n}{ }^{2}=\frac{(n+1) \bar{\omega}}{2} \cdot\left[\sqrt{(n+1)^{2} \bar{\omega}^{2}+1}-(n+1) \bar{\omega}\right]
\end{aligned} .
$$

Then

$$
\begin{aligned}
\left|\bar{k}_{n}\right|^{2}=\frac{(n+1) \bar{\omega}}{2} \cdot\left[\sqrt{(n+1)^{2} \bar{\omega}^{2}+1}+(n+1) \bar{\omega}\right] \\
+\frac{(n+1) \bar{\omega}}{2} \cdot\left[\sqrt{(n+1)^{2} \bar{\omega}^{2}+1}-(n+1) \bar{\omega}\right],
\end{aligned}
$$

or

$$
\left|\bar{k}_{n}\right|^{2}=\frac{(n+1) \bar{\omega}}{2} \cdot 2 \sqrt{(n+1)^{2} \bar{\omega}^{2}+1} .
$$

Taking square roots gives

$$
\left|\bar{k}_{n}\right|=\sqrt{(n+1) \bar{\omega} \cdot \sqrt{(n+1)^{2} \bar{\omega}^{2}+1}} .
$$

Thus,

$$
\bar{v}_{\mathrm{ph}, n}(\bar{\omega})=\frac{\bar{\omega}}{\sqrt{(n+1) \bar{\omega} \cdot \sqrt{(n+1)^{2} \bar{\omega}^{2}+1}}}=\sqrt{\frac{\bar{\omega}}{n+1}} \cdot \frac{1}{\sqrt{\sqrt{(n+1)^{2} \bar{\omega}^{2}+1}}} .
$$


Figure 7 shows plots of the phase speeds as functions of the frequency for several modes. Here, we can see that the phase speed increases rapidly with $\bar{\omega}$ for small frequencies but begins to "flatten out" as $\bar{\omega}$ increases approaching a positive constant. This constant is different for each wavenumber with a maximum of 1.0 for $n=0$ and decreasing for increasing $n$.

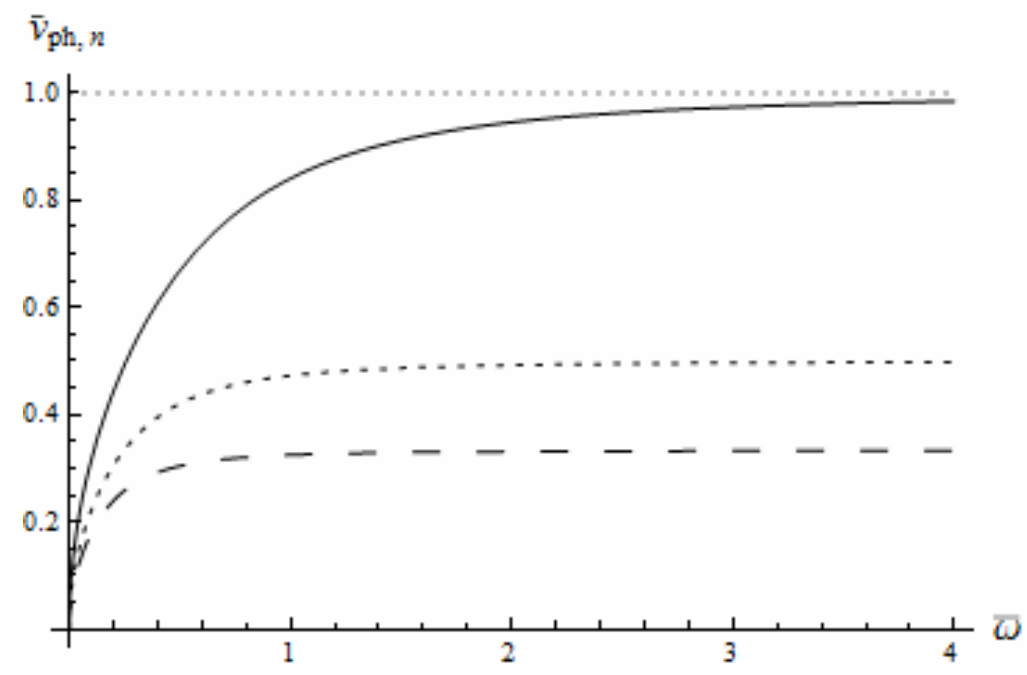

Figure 7. The phase speeds plotted against frequency for $n=0$ (solid), 1 (dotted) and 2 (dashed).

\section{Low-Frequency Region}

Using the low and high frequency approximations for $\bar{\alpha}_{n}$ and $\bar{\beta}_{n}$ given by Equation and Equation, respectively, we have

$$
\left|\bar{k}_{n}\right|^{2} \approx \frac{(n+1) \bar{\omega}}{2}+\frac{(n+1) \bar{\omega}}{2}=(n+1) \bar{\omega} .
$$


Then $\left|\bar{k}_{n}\right| \approx \sqrt{(n+1) \bar{\omega}}$ and so the low frequency approximation for the phase speeds

are given by

$$
\bar{v}_{\mathrm{ph}, \mathrm{n}}(\bar{\omega}) \approx \frac{\bar{\omega}}{\sqrt{(n+1) \bar{\omega}}}=\sqrt{\frac{\bar{\omega}}{n+1}}
$$

Figure 3 shows a comparison of the phase speed in the low-frequency region with the approximation given by Equation (3.3.4).

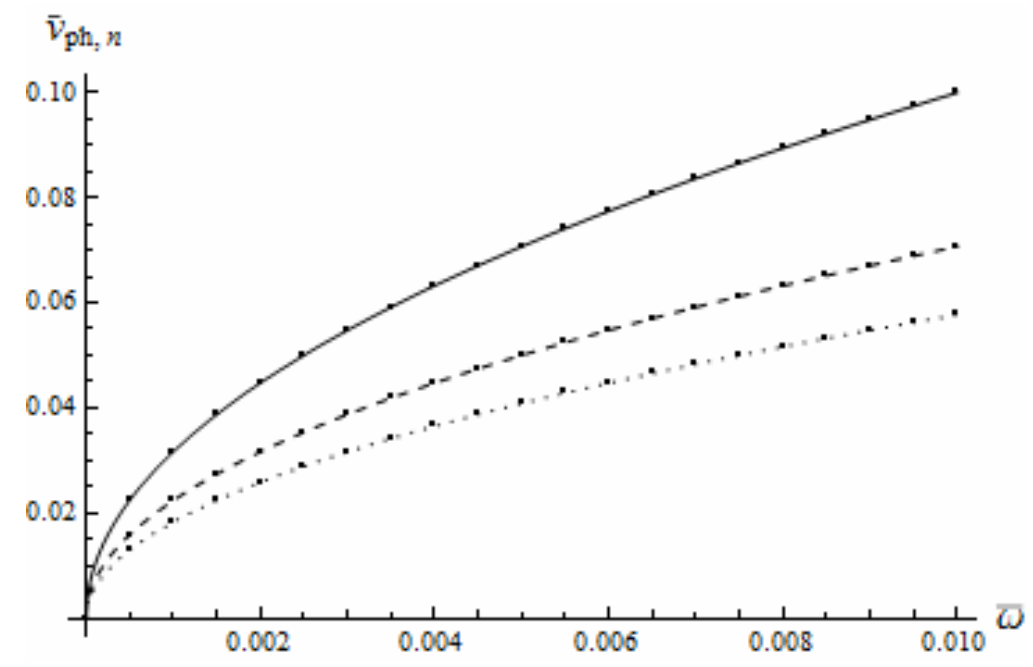

Figure 8. The phase speeds plotted against frequency for $n=0$ (solid), 1 (small dotted) and 2 (dashed) in the low-frequency region. Note that agreement with corresponding analytic solution given by Equation (3.3.5). 


\section{High-Frequency Region}

Using the low and high frequency approximations for $\bar{\alpha}_{n}$ and $\bar{\beta}_{n}$ given by Equation and Equation, respectively, we have

$$
\left|\bar{k}_{n}\right|^{2} \approx(n+1)^{2} \bar{\omega}^{2}+\frac{1}{4}
$$

or, for $\bar{\omega} \gg 1$,

$$
\left|\bar{k}_{n}\right|^{2} \approx(n+1)^{2} \bar{\omega}^{2} .
$$

Then, for high frequencies, $\left|\bar{k}_{n}\right| \approx(n+1) \bar{\omega}$ and so the high frequency approximation for the phase speeds are given by

$$
\bar{v}_{\mathrm{ph}, \mathrm{n}}(\bar{\omega}) \approx \frac{\bar{\omega}}{(n+1) \bar{\omega}}=\frac{1}{n+1} .
$$

Figure 4 shows a comparison of the phase speed in the low-frequency region with the approximation given by Equation (3.3.5). 


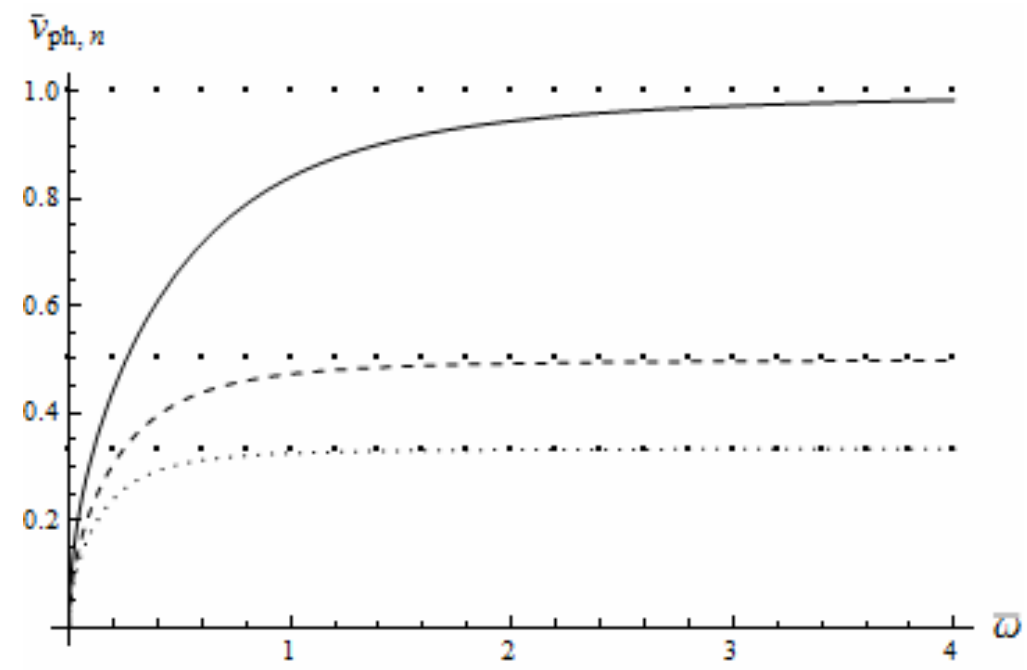

Figure 9. The phase speeds plotted against frequency for $n=0$ (solid), 1 (dashed) and 2 (dotted) in the highfrequency region. Note that agreement with corresponding analytic solution given by Equation (3.3.6). 


\section{Chapter 4. Determining the Peak of the First-Order Correction}

\section{Term and Constraints on the Perturbation Parameter}

\subsection{Overview}

In this Chapter, we first investigate the behavior of the modulus of the first-order correction $\left|\overline{\mathcal{P}_{1}}\right|$. From Figure 2, we see that $\left|\overline{\mathcal{P}_{1}}\right|$ is bounded and has a unique maximum, which we denote by $\bar{x}_{\max }$. Thus, we study the behavior of $\bar{x}_{\max }$ in terms of frequency. Next, we determine bounds on the perturbation parameter $\varepsilon$ that ensures convergence of the solution $\bar{P}$.

\subsection{Maximizing the Modulus of the First-Order Correction}

Here, we seek the value of $\bar{x}$ for which $\left|\overline{\mathcal{P}_{1}}\right|$ is a maximum. Let $\bar{x}_{\max }$ denote this quantity. From Equation (2.5.5) the first-order approximation is given by

$$
\overline{\mathcal{P}_{1}}(\bar{x}, \bar{t})=-\frac{1}{8} \cdot\left[e^{-\bar{\beta}_{1} \bar{x}} \cdot e^{i\left(\bar{\alpha}_{1} \bar{x}-2 \bar{\omega} \bar{t}\right)}-e^{-2 \bar{\beta}_{0} \bar{x}} \cdot e^{2 i\left(\overline{\alpha_{0}} \bar{x}-\bar{\omega} \bar{t}\right)}\right],
$$

where the wavenumbers have been expressed in terms of their real and imaginary parts $\bar{\alpha}_{n}$ and $\bar{\beta}_{n}$. The square of the modulus is given by $\left|\overline{\mathcal{P}_{1}}\right|=\sqrt{\overline{\mathcal{P}_{1}^{*}} \cdot \overline{\mathcal{P}_{1}}}$. Conjugating Equation (4.2.1) gives

$$
\overline{\mathcal{P}_{1}^{*}}(\bar{x}, \bar{t})=-\frac{1}{8} \cdot\left[e^{-\bar{\beta}_{1} \bar{x}} \cdot e^{-i\left(\bar{\alpha}_{1} \bar{x}-2 \bar{\omega} \bar{t}\right)}-e^{-2 \bar{\beta}_{0} \bar{x}} \cdot e^{-2 i\left(\overline{\alpha_{0}} \bar{x}-\bar{\omega} \bar{t}\right)}\right] .
$$


Then

$$
\left|\overline{\mathcal{P}_{1}}\right|^{2}=\frac{1}{64} \cdot\left(e^{-\bar{\beta}_{1} \bar{x}} \cdot e^{-i \bar{\alpha}_{1} \bar{x}}-e^{-2 \bar{\beta}_{0} \bar{x}} \cdot e^{-2 i \bar{\alpha}_{0} \bar{x}}\right) \cdot\left(e^{-\bar{\beta}_{1} \bar{x}} \cdot e^{i \bar{\alpha}_{1} \bar{x}}-e^{-2 \bar{\beta}_{0} \bar{x}} \cdot e^{2 i \bar{\alpha}_{0} \bar{x}}\right)
$$

or

$$
\left|\overline{\mathcal{P}}_{1}\right|^{2}=\frac{1}{64} \cdot\left[e^{-2 \bar{\beta}_{1} \bar{x}}+e^{-4 \bar{\beta}_{0} \bar{x}}-e^{-\left(\bar{\beta}_{1}+2 \bar{\beta}_{0}\right) \bar{x}} \cdot e^{-i\left(\bar{\alpha}_{1}-2 \bar{\alpha}_{0}\right) \bar{x}}-e^{-\left(\bar{\beta}_{1}+2 \bar{\beta}_{0}\right) \bar{x}} \cdot e^{i\left(\bar{\alpha}_{1}-2 \bar{\alpha}_{0}\right) \bar{x}}\right]
$$

or

$$
\left|\overline{\mathcal{P}_{1}}\right|^{2}=\frac{1}{64} \cdot\left\{e^{-2 \overline{\beta_{1}} \bar{x}}+e^{-4 \bar{\beta}_{0} \bar{x}}-e^{-\left(\bar{\beta}_{1}+2 \bar{\beta}_{0}\right) \bar{x}} \cdot\left[e^{i\left(\bar{\alpha}_{1}-2 \bar{\alpha}_{0}\right) \bar{x}}+e^{-i\left(\bar{\alpha}_{1}-2 \bar{\alpha}_{0}\right) \bar{x}}\right]\right\} .
$$

Now, using the identity $\cos x=\frac{1}{2}\left(e^{i x}+e^{-i x}\right)$, Equation (4.2.3) becomes

$$
\left|\overline{\mathcal{P}_{1}}\right|^{2}=\frac{1}{64} \cdot\left[e^{-2 \bar{\beta}_{1} \bar{x}}+e^{-4 \bar{\beta}_{0} \bar{x}}-e^{-\left(\bar{\beta}_{1}+2 \bar{\beta}_{0}\right) \bar{x}} \cos \left(\bar{\alpha}_{1}-2 \bar{\alpha}_{0}\right) \bar{x}\right] .
$$

Factoring $e^{-\left(\bar{\beta}_{1}+2 \bar{\beta}_{0}\right) \bar{x}}$ from Equation (4.2.4) gives

$$
\left|\overline{\mathcal{P}_{1}}\right|^{2}=\frac{e^{-\left(\bar{\beta}_{1}+2 \bar{\beta}_{0}\right) \bar{x}}}{64} \cdot\left[e^{-\left(\bar{\beta}_{1}-2 \bar{\beta}_{0}\right) \bar{x}}+e^{\left(\bar{\beta}_{1}-2 \bar{\beta}_{0}\right) \bar{x}}-2 \cos \left(\bar{\alpha}_{1}-2 \bar{\alpha}_{0}\right) \bar{x}\right]
$$

Now, using the identity $\cosh x=\frac{1}{2}\left(e^{x}+e^{-x}\right)$, Equation (4.2.5) becomes

$$
\left|\overline{\mathcal{P}_{1}}\right|^{2}=\frac{e^{-\left(\bar{\beta}_{1}+2 \bar{\beta}_{0}\right) \bar{x}}}{64} \cdot\left[2 \cosh \left(\bar{\beta}_{1}-2 \bar{\beta}_{0}\right) \bar{x}-2 \cos \left(\bar{\alpha}_{1}-2 \bar{\alpha}_{0}\right) \bar{x}\right]
$$


Thus, the modulus of the first-order correction becomes

$$
\left|\overline{\mathcal{P}}_{1}\right|=\frac{e^{-\frac{1}{2}\left(\bar{\beta}_{1}+2 \bar{\beta}_{0}\right) \bar{x}}}{4 \sqrt{2}} \cdot \sqrt{\cosh \left(\bar{\beta}_{1}-2 \bar{\beta}_{0}\right) \bar{x}-\cos \left(\bar{\alpha}_{1}-2 \bar{\alpha}_{0}\right) \bar{x}}
$$

To find the location of the peak of $\left|\overline{\mathcal{P}_{1}}\right|$, we must differentiate $\left|\overline{\mathcal{P}_{1}}\right|$ with respect to $\bar{x}$.

The peak of $\left|\overline{\mathcal{P}_{1}}\right|$, which we denote by $\bar{x}_{\text {max }}$, is a zero of $d\left|\overline{\mathcal{P}_{1}}\right| / d \bar{x}$. From Equation (4.2.7) we have

$$
\frac{d\left|\bar{P}_{1}\right|}{d \bar{x}}=-\frac{e^{-\frac{\bar{\delta}}{2} \bar{x}}}{8 \sqrt{2}} \cdot\left(\bar{\delta} \sqrt{\cosh \bar{\gamma} \bar{x}-\cos \bar{\lambda} \bar{x}}-\frac{\bar{\gamma} \sinh \bar{\gamma} \bar{x}+\bar{\lambda} \sin \bar{\lambda} \bar{x}}{\sqrt{\cosh \bar{\gamma} \bar{x}-\cos \bar{\lambda} \bar{x}}}\right)
$$

where $\bar{\delta}=\bar{\beta}_{1}+2 \bar{\beta}_{0}, \bar{\gamma}=\bar{\beta}_{1}-2 \bar{\beta}_{0}$ and $\bar{\lambda}=\bar{\alpha}_{1}-2 \bar{\alpha}_{0}$. In order for Equation (4.2.8) to vanish, we must have

$$
\bar{\delta} \sqrt{\cosh \bar{\gamma} \bar{x}-\cos \bar{\lambda} \bar{x}}-\frac{\bar{\gamma} \sinh \bar{\gamma} \bar{x}+\bar{\lambda} \sin \bar{\lambda} \bar{x}}{\sqrt{\cosh \bar{\gamma} \bar{x}-\cos \bar{\lambda} \bar{x}}}=0
$$

or

$$
\bar{\delta} \cosh \bar{\gamma} \bar{x}-\bar{\gamma} \sinh \bar{\gamma} \bar{x}=\bar{\delta} \cos \bar{\lambda} \bar{x}+\bar{\lambda} \sin \bar{\lambda} \bar{x}
$$

The LHS of Equation (4.2.9) can be written as

$$
\bar{\delta} \cosh \bar{\gamma} \bar{x}-\bar{\gamma} \sinh \bar{\gamma} \bar{x}=\bar{C}_{1} \cosh \left(\bar{\gamma} \bar{x}-\bar{\kappa}_{1}\right)=\bar{C}_{1} \cosh \bar{\kappa}_{1} \cdot \cosh \bar{\gamma} \bar{x}-\bar{C}_{1} \sinh \bar{\kappa}_{1} \sinh \bar{\gamma} \bar{x}
$$

Then, we must have

$$
\begin{aligned}
& \bar{\delta}=\bar{C}_{1} \cosh \bar{\kappa}_{1} \\
& \bar{\gamma}=\bar{C}_{1} \sinh \bar{\kappa}_{1}
\end{aligned}
$$


Dividing the second equation in (4.2.10) by the first gives

$$
\frac{\bar{\gamma}}{\bar{\delta}}=\tanh \bar{\kappa}_{1}
$$

Moreover, subtracting the square of the second equation in (4.2.10) from the square of the first gives

$$
\bar{\delta}^{2}-\bar{\gamma}^{2}=\bar{C}_{1}^{2}\left(\cosh ^{2} \bar{\kappa}_{1}-\sinh ^{2} \bar{\kappa}_{1}\right)
$$

Thus, from Equations (4.2.11) and (4.2.12), and using the identity $\cosh ^{2} x-\sinh ^{2} x=1$, we have

$$
\begin{gathered}
\bar{\kappa}_{1}=\tanh ^{-1} \frac{\bar{\gamma}}{\bar{\delta}} \\
\bar{C}_{1}=\sqrt{\bar{\delta}^{2}-\bar{\gamma}^{2}}
\end{gathered} .
$$

Similarly, the RHS of Equation (4.2.9) can be written as

$$
\bar{\lambda} \sin \bar{\lambda} \bar{x}+\bar{\delta} \cos \bar{\lambda} \bar{x}=\bar{C}_{2} \cos \left(\bar{\lambda} \bar{x}-\bar{\kappa}_{2}\right)=\bar{C}_{2} \cos \bar{\kappa}_{2} \cos \bar{\lambda} \bar{x}+\bar{C}_{2} \sin \bar{\kappa}_{2} \sin \bar{\lambda} \bar{x}
$$

Then, we must have

$$
\begin{aligned}
& \bar{\lambda}=\bar{C}_{2} \sin \bar{\kappa}_{2} \\
& \bar{\delta}=\bar{C}_{2} \cos \bar{\kappa}_{2} .
\end{aligned}
$$

Dividing the first equation in (4.2.14) by the second gives

$$
\frac{\bar{\lambda}}{\bar{\delta}}=\tan \bar{\kappa}_{2} .
$$


Moreover, adding the square of the first equation in (4.2.14) to the square of the second gives

$$
\bar{\lambda}^{2}+\bar{\delta}^{2}=\bar{C}_{2}^{2}\left(\sin ^{2} \bar{\kappa}_{2}+\cos ^{2} \bar{\kappa}_{2}\right)
$$

Thus, from Equations (4.2.15) and (4.2.16), and using the identity $\sin ^{2} x+\cos ^{2} x=1$, we have

$$
\begin{gathered}
\bar{\kappa}_{2}=\tan ^{-1} \frac{\bar{\lambda}}{\bar{\delta}} \\
\bar{C}_{2}=\sqrt{\bar{\lambda}^{2}+\bar{\delta}^{2}} .
\end{gathered}
$$

Thus, Equation (4.2.9) can be written as

$$
\bar{C}_{1} \cosh \left(\bar{\gamma} \bar{x}-\bar{\kappa}_{1}\right)=\bar{C}_{2} \cos \left(\bar{\lambda} \bar{x}-\bar{\kappa}_{2}\right)
$$

where $\bar{\kappa}_{1}, \bar{C}_{1}, \bar{\kappa}_{2}$ and $\bar{C}_{2}$ are given by Equations (4.2.13) and (4.2.17). Finally, letting $\bar{C}=\bar{C}_{2} / \bar{C}_{1}$, Equation (4.2.18) becomes

$$
\cosh \left(\bar{\gamma} \bar{x}_{\max }-\bar{\kappa}_{1}\right)=\bar{C} \cos \left(\bar{\lambda}_{\max }-\bar{\kappa}_{2}\right)
$$

\section{Low-Frequency Region}

From Chapter 3, we have, for the low-frequency regime,

$$
\begin{aligned}
& \bar{\alpha}_{0}=\bar{\beta}_{0}=\sqrt{\frac{\bar{\omega}}{2}} \\
& \bar{\alpha}_{1}=\bar{\beta}_{1}=\sqrt{\bar{\omega}}
\end{aligned}
$$


Then $\bar{\delta}, \bar{\gamma}$ and $\bar{\lambda}$, become

$$
\begin{gathered}
\bar{\delta}=\bar{\beta}_{1}+2 \bar{\beta}_{0}=\sqrt{\bar{\omega}}+2 \sqrt{\frac{\bar{\omega}}{2}}=\left(1+\frac{2}{\sqrt{2}}\right) \sqrt{\bar{\omega}}=(1+\sqrt{2}) \sqrt{\bar{\omega}} \\
\bar{\gamma}=\bar{\beta}_{1}-2 \bar{\beta}_{0}=\sqrt{\bar{\omega}}-2 \sqrt{\frac{\bar{\omega}}{2}}=\left(1-\frac{2}{\sqrt{2}}\right) \sqrt{\bar{\omega}}=(1-\sqrt{2}) \sqrt{\bar{\omega}}, \\
\bar{\gamma}=\bar{\alpha}_{1}-2 \bar{\alpha}_{0}=\sqrt{\bar{\omega}}-2 \sqrt{\frac{\bar{\omega}}{2}}=\left(1-\frac{2}{\sqrt{2}}\right) \sqrt{\bar{\omega}}=(1-\sqrt{2}) \sqrt{\bar{\omega}}
\end{gathered}
$$

respectively. Thus, letting $\bar{y}=(1-\sqrt{2}) \sqrt{\bar{\omega}} \bar{x}$, we have, from Equation (4.2.9),

$$
(1+\sqrt{2}) \cosh \bar{y}-(1-\sqrt{2}) \sinh \bar{y}=(1-\sqrt{2}) \sin \bar{y}+(1+\sqrt{2}) \cos \bar{y}
$$

The solutions of Equation (6.1.1) are given in Table 1 for some small frequencies $\bar{\omega}$ as well as the product $\sqrt{\bar{\omega}} \bar{x}_{\max }$. Note that for decreasing frequency, the product $\sqrt{\bar{\omega}} \bar{x}_{\max }$ converges to the value $2(\sqrt{2}-1) \approx 0.828$. Thus, for small frequencies, we have

$$
\bar{x}_{\max } \approx \frac{0.828}{\sqrt{\bar{\omega}}} .
$$

\begin{tabular}{|c|c|c|}
\hline $\bar{\omega}$ & $\bar{x}_{\max }$ & $\sqrt{\bar{\omega}} \bar{x}_{\max }$ \\
\hline 0.0001 & 82.8551857057 & 0.8285518571 \\
\hline 0.0002 & 58.5917705348 & 0.8286127653 \\
\hline 0.0003 & 47.8434971211 & 0.8286736783 \\
\hline 0.0004 & 41.4367297906 & 0.8287345958 \\
\hline 0.0005 & 37.0648623540 & 0.8287955180 \\
\hline 0.0006 & 33.8379226641 & 0.8288564448 \\
\hline 0.0007 & 31.3301319297 & 0.8289173763 \\
\hline 0.0008 & 29.3088093070 & 0.8289783124 \\
\hline 0.0009 & 27.6346417700 & 0.8290392531 \\
\hline 0.001 & 26.2184503560 & 0.8291001984 \\
\hline
\end{tabular}

Table 1. The product $\sqrt{\bar{\omega}} \bar{x}_{\max }$ for various values of $\bar{\omega}$ and the corresponding values of $\bar{x}_{\max }$ in the lowfrequency region. 


\section{High-Frequency Region}

From Chapter 3, we have, for the low-frequency regime,

$$
\begin{gathered}
\bar{\alpha}_{0}=\bar{\omega} \\
\bar{\alpha}_{1}=2 \bar{\omega} \\
\bar{\beta}_{0}=\bar{\beta}_{1}=\frac{1}{2}
\end{gathered}
$$

Then $\bar{\delta}, \bar{\gamma}$ and $\bar{\lambda}$, become

$$
\begin{gathered}
\bar{\delta}=\bar{\beta}_{1}+2 \bar{\beta}_{0}=\frac{1}{2}+2 \cdot \frac{1}{2}=\frac{1}{2}+1=\frac{3}{2} \\
\bar{\gamma}=\bar{\beta}_{1}-2 \bar{\beta}_{0}=\frac{1}{2}-2 \cdot \frac{1}{2}=\frac{1}{2}-1=-\frac{1}{2} . \\
\bar{\gamma}=\bar{\alpha}_{1}-2 \bar{\alpha}_{0}=2 \bar{\omega}-2 \bar{\omega}=0
\end{gathered}
$$

respectively. Thus, Equation (4.2.9) becomes

$$
\frac{3}{2} \cosh \left(-\frac{\bar{x}}{2}\right)+\frac{1}{2} \sinh \left(-\frac{\bar{x}}{2}\right)=\frac{3}{2}
$$

Exploiting the parity of the hyperbolic cosine and sine functions and multiplying by 2, Equation (4.2.26) can be written

$$
3 \cosh \frac{\bar{x}}{2}-\sinh \frac{\bar{x}}{2}=3
$$

Using the identities $\cosh x=\frac{1}{2}\left(e^{x}+e^{-x}\right)$ and $\sinh x=\frac{1}{2}\left(e^{x}-e^{-x}\right)$, Equation (4.2.27) becomes

$$
\frac{3}{2}\left(e^{\frac{\bar{x}}{2}}+e^{-\frac{\bar{x}}{2}}\right)-\frac{1}{2}\left(e^{\frac{\bar{x}}{2}}-e^{-\frac{\bar{x}}{2}}\right)=3
$$


or, multiplying $2 e^{\frac{\bar{x}}{2}} 2$ and expanding,

$$
2 e^{\bar{x}}+4=6 e^{\frac{\bar{x}}{2}},
$$

or, re-writing

$$
e^{\bar{x}}-3 e^{\frac{\bar{x}}{2}}+2=0
$$

From the Quadratic Formula, we find

$$
e^{\frac{\bar{x}}{2}}=\frac{3+\sqrt{9-4 \cdot 2}}{2}=\frac{3+\sqrt{9-8}}{2}=\frac{3+\sqrt{1}}{2}=\frac{4}{2}=2 .
$$

Taking the natural logarithm of Equation (4.2.28) gives

$$
\frac{\bar{x}}{2}=\ln 2 .
$$

Thus, in the high-frequency region, the location of the peak of $\left|\overline{\mathcal{P}_{1}}\right|$ is given by

$$
\bar{x}=2 \ln 2
$$

A plot of $\bar{x}_{\max }$ versus frequency is shown in Figure 10. The limiting values in the low and highfrequency limits are shown as dashed lines. 


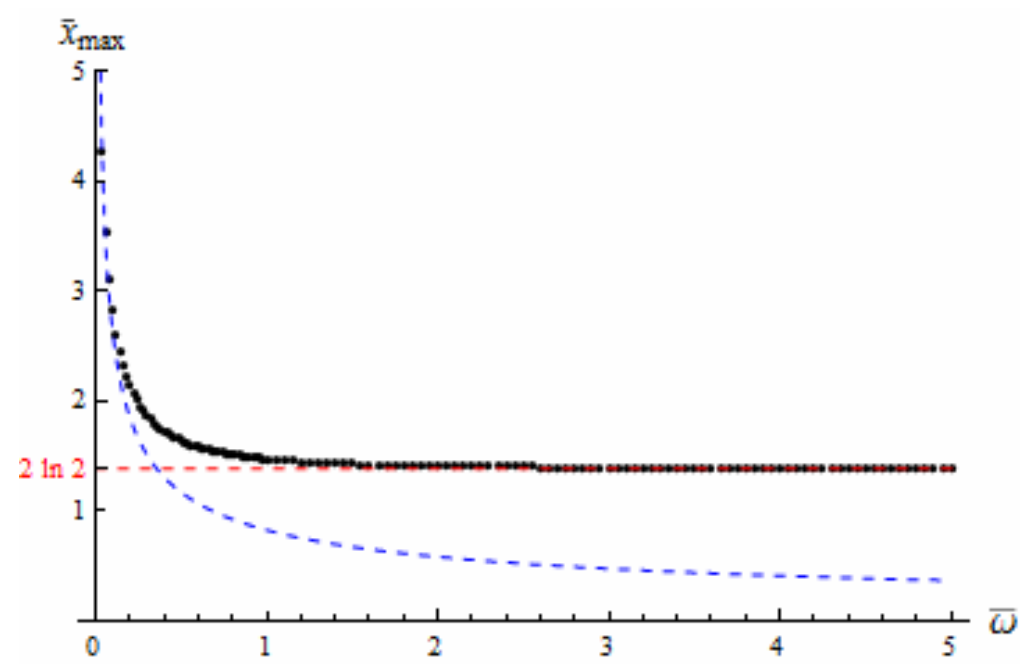

Figure 10. The response of location of the peak of the modulus of the first-order correction to frequency. The low-frequency limit $2(\sqrt{2}-1) / \sqrt{\bar{\omega}}$ is shown as a blue dashed line and the high-frequency limit $2 \ln 2$ is shown as a red dashed line.

\subsection{Determining the Constraints on the Perturbation Parameter}

Here, we wish to examine the constraints on the perturbation parameter $\varepsilon$; that is, we seek the values of $\varepsilon$ so as to ensure proper convergence. Using the definition of $\left|\overline{\mathcal{P}_{1}}\right|$, we have, from the Triangle Inequality,

$$
|\overline{\mathcal{P}}| \leq|| \overline{\mathcal{P}_{0}} \mid \cdot\left(1+\varepsilon \frac{\left|\overline{\mathcal{P}_{1}}\right|}{\left|\overline{\mathcal{P}_{0}}\right|}+\varepsilon^{2} \frac{\left|\overline{\mathcal{P}_{2}}\right|}{\left|\overline{\mathcal{P}_{0}}\right|}\right)
$$

Thus, we wish to satisfy the following two inequalities:

$$
\begin{gathered}
\varepsilon \frac{\left|\overline{\mathcal{P}_{1}}\right|}{\left|\overline{\mathcal{P}_{0}}\right|} \ll 1 \\
\varepsilon^{2} \frac{\left|\overline{\mathcal{P}_{2}}\right|}{\left|\overline{\mathcal{P}_{0}}\right|} \ll 1
\end{gathered},
$$


where $\left|\overline{\mathcal{P}_{0}}\right|,\left|\overline{\mathcal{P}_{1}}\right|$ and $\left|\overline{\mathcal{P}_{2}}\right|$ are all evaluated at $\bar{x}_{\text {max }}$. Thus, letting $\tau \ll 1$ denote some predetermined tolerance, for example $\tau=0.1$, we have

$$
\begin{gathered}
\varepsilon \frac{\left|\overline{\mathcal{P}_{1}}\right|}{\left|\overline{\mathcal{P}_{0}}\right|}=\tau \\
\varepsilon^{2} \frac{\left|\overline{\mathcal{P}_{2}}\right|}{\left|\overline{\mathcal{P}_{0}}\right|}=\tau^{2}
\end{gathered}
$$

The plots of $\ln \left(\left|\overline{\mathcal{P}_{1}}\right| /\left|\overline{\mathcal{P}_{0}}\right|\right)$ and $\ln \left(\left|\overline{\mathcal{P}_{2}}\right| /\left|\overline{\mathcal{P}_{0}}\right|\right)$ versus $\ln \bar{\omega}$ are shown in Figure 11. From these plots, we can see that these ratios remain bounded at all frequencies. For example, for a tolerance $\tau=0.1$,

$$
\begin{gathered}
\varepsilon \frac{\left|\overline{\mathcal{P}_{1}}\right|}{\left|\overline{\mathcal{P}_{0}}\right|}=0.1 \\
\varepsilon^{2} \frac{\left|\overline{\mathcal{P}_{2}}\right|}{\left|\overline{\mathcal{P}_{0}}\right|}=0.01
\end{gathered}
$$




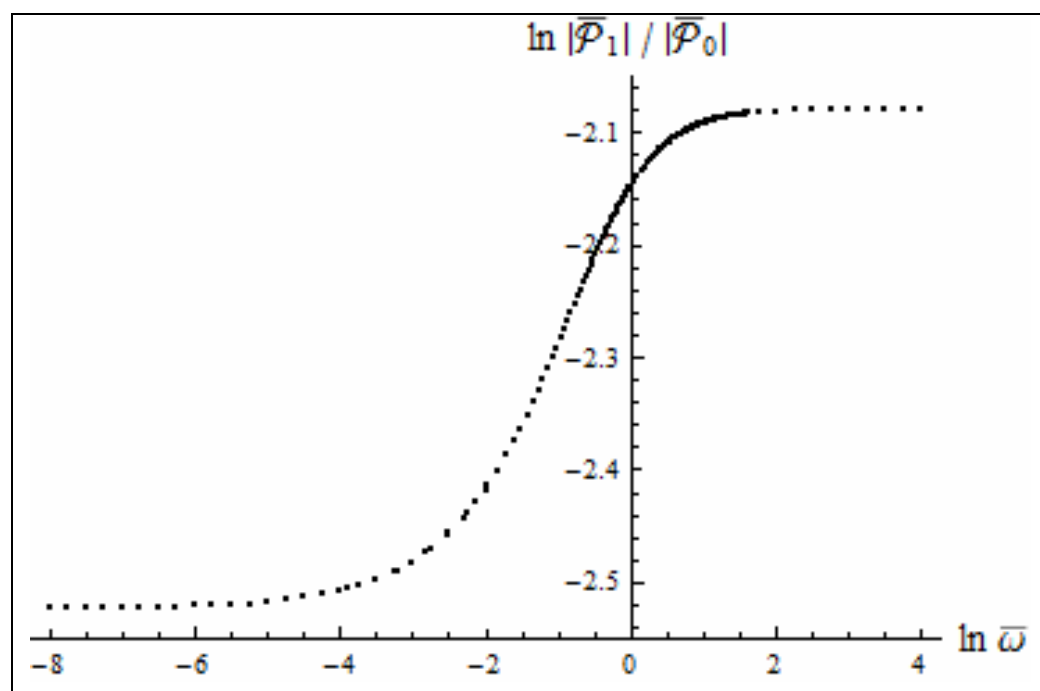

(a)

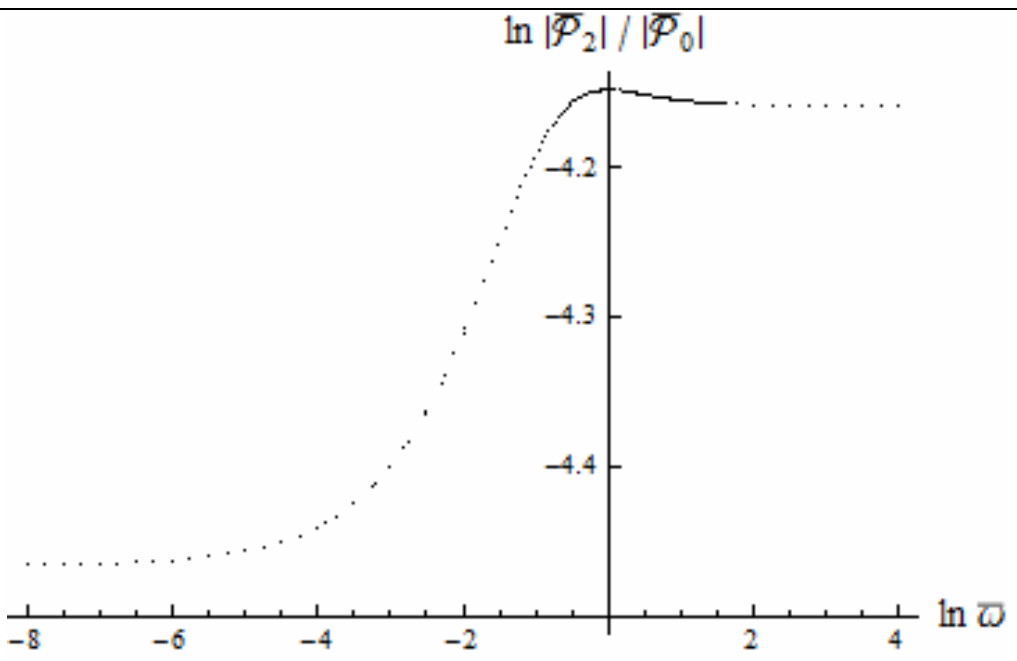

(b)

Figure 12. A log-log plot of the ratio of (a) $\left|\overline{\mathcal{P}_{1}}\right| /\left|\overline{\mathcal{P}_{0}}\right|$ and (b) $\left|\overline{\mathcal{P}_{2}}\right| /\left|\overline{\mathcal{P}_{0}}\right|$ to versus frequency. Taken together, these two plots, define the range over which $\varepsilon$ may vary so that convergence of $\bar{P}$ will occur. We seek an maximum value of $\varepsilon$ that satisfies both convergence conditions. 
We must choose a value of $\varepsilon$ that satisfies both equations in (4.3.2). Letting $M_{1} \in\left\{\left|\bar{P}_{1}\right| /\left|\bar{P}_{0}\right|\right\}$ and $M_{2} \in\left\{\left|\bar{P}_{2}\right| /\left|\bar{P}_{0}\right|\right\}$. Then, we find using Figure 11, that the bounds on $M_{1}$ and $M_{2}$ are $e^{-2.53}<M_{1}<e^{-2.08}$ and $e^{-4.47}<M_{2}<e^{-4.159}$, respectively. This results in the corresponding bounds on $\varepsilon: 0.8<\varepsilon<1.25$ and $0.8<\varepsilon<0.957$, for $M_{1}$ and $M_{2}$, respectively. Next we need to choose a value of epsilon from these bounds that satisfies both inequalities. A choice of $\varepsilon \leq \varepsilon_{\max }$, where $\varepsilon_{\max }=0.8$ satisfies both inequalities in the low and high-frequency regions. 


\section{Chapter 5. Details of Perturbation Solution}

\subsection{Overview}

In this chapter, we derive formulas for the low and high-frequency limits of $\bar{P}_{0}, \bar{P}_{1}$ and $\bar{P}_{2}$. We then compare these approximations with the solution obtained in Chapter 2 in Figures 8 and 9. The dots in the figures denote the approximations obtained in Sections 5.2-5.4.

\subsection{Zeroth-Order Term}

Using Equation (2.4.2) the zeroth-order solution $\bar{P}_{0}$ is

$$
\bar{P}_{0}(\bar{x}, \bar{t})=\frac{i}{2} e^{i\left[\left(\bar{\alpha}_{0}+i \bar{\beta}_{0}\right) \bar{x}-\bar{\omega} \bar{t}\right]}+\text { c.c. }
$$

or

$$
\bar{P}_{0}(\bar{x}, \bar{t})=\frac{i}{2} e^{-\bar{\beta}_{0} \bar{x}} e^{i\left(\bar{\alpha}_{0} \bar{x}-\bar{\omega} \bar{t}\right)}+\text { c.c. }
$$

or

$$
\bar{P}_{0}(\bar{x}, \bar{t})=\frac{i}{2} e^{-\bar{\beta}_{0} \bar{x}} e^{i\left(\bar{\alpha}_{0} \bar{x}-\bar{\omega} \bar{t}\right)}-\frac{i}{2} e^{-\bar{\beta}_{0} \bar{x}} e^{-i\left(\bar{\alpha}_{0} \bar{x}-\bar{\omega} \bar{t}\right)}
$$

or

$$
\bar{P}_{0}(\bar{x}, \bar{t})=-e^{-\bar{\beta}_{0} \bar{x}} \cdot \frac{e^{i\left(\bar{\alpha}_{0} \bar{x}-\bar{\omega} \bar{t}\right)}-e^{-\bar{\beta}_{0} \bar{x}} e^{-i\left(\bar{\alpha}_{0} \bar{x}-\bar{\omega} \bar{t}\right)}}{2 i}
$$


Thus

$$
\bar{P}_{0}(\bar{x}, \bar{t})=-e^{-\bar{\beta}_{0} \bar{x}} \sin \left(\bar{\alpha}_{0} \bar{x}-\bar{\omega} \bar{t}\right)
$$

\subsection{The First-Order Correction}

Using Equation (2.5.5) the first-order correction $\bar{P}_{1}$ is

$$
\bar{P}_{1}(\bar{x}, \bar{t})=\frac{i}{2} \cdot\left(\frac{i}{4} e^{i\left(\bar{k}_{1} \bar{x}-2 \bar{\omega} \bar{t}\right)}-\frac{i}{4} e^{2 i\left(\bar{k}_{0} \bar{x}-\bar{\omega} \bar{t}\right)}\right)+\text { c.c. },
$$

or

$$
\bar{P}_{1}(\bar{x}, \bar{t})=\left(\frac{i}{2}\right)^{2} \cdot \frac{1}{2}\left[e^{i\left(\bar{k}_{1} \bar{x}-2 \bar{\omega} \bar{t}\right)}-e^{2 i\left(\bar{k}_{0} \bar{x}-\bar{\omega} \bar{t}\right)}\right]+\text { c.c. }
$$

or

$$
\bar{P}_{1}(\bar{x}, \bar{t})=\left(\frac{i}{2}\right)^{2} \cdot \frac{1}{2}\left[e^{-\bar{\beta}_{1} \bar{x}} \cdot e^{i\left(\bar{\alpha}_{1} \bar{x}-2 \bar{\omega} \bar{t}\right)}-e^{-2 \bar{\beta}_{0} \bar{x}} e^{2 i\left(\bar{\alpha}_{0} \bar{x}-\bar{\omega} \bar{t}\right)}\right]+\text { c.c. },
$$

or

$$
\bar{P}_{1}(\bar{x}, \bar{t})=-\frac{e^{-\bar{\beta}_{1} \bar{x}}}{8}\left[e^{i\left(\bar{\alpha}_{1} \bar{x}-2 \bar{\omega} \bar{t}\right)}+e^{-i\left(\bar{\alpha}_{1} \bar{x}-2 \bar{\omega} \bar{t}\right)}\right]-\frac{e^{-2 \bar{\beta}_{0} \bar{x}}}{8}\left[e^{2 i\left(\bar{\alpha}_{0} \bar{x}-\bar{\omega} \bar{t}\right)}+e^{-2 i\left(\bar{\alpha}_{0} \bar{x}-\bar{\omega} \bar{t}\right)}\right],
$$

or

$$
\bar{P}_{1}(\bar{x}, \bar{t})=-\frac{1}{4} \cdot\left[e^{-\bar{\beta}_{1} \bar{x}} \cos \left(\bar{\alpha}_{1} \bar{x}-2 \bar{\omega} \bar{t}\right)-e^{-2 \bar{\beta}_{0} \bar{x}} \cos 2\left(\bar{\alpha}_{0} \bar{x}-\bar{\omega} \bar{t}\right)\right] .
$$




\subsection{The Second-Order Correction}

Using Equation (2.6.10) the second-order correction $\bar{P}_{2}$ is

$$
\bar{P}_{2}(\bar{x}, \bar{t})=\left(\frac{i}{2}\right)^{3} \cdot \frac{1}{4}\left[\bar{A}_{2}^{\prime} e^{i \bar{k}_{2} \bar{x}-3 \bar{\omega} \bar{t}}+\bar{B}_{2}^{\prime} e^{i\left(\bar{k}_{0}+\bar{k}_{1}\right) \bar{x}-3 \bar{\omega} \bar{t}}+e^{3 i \bar{k}_{0} \bar{x}-3 \bar{\omega} \bar{t}}\right]+\text { c.c. }
$$

where $\bar{A}_{2}^{\prime}$ and $\bar{B}_{2}^{\prime}$ are given by Equation (2.6.11) and Equation (2.6.12), respectively. Then

$$
\begin{aligned}
32 \bar{P}_{2}(\bar{x}, \bar{t})=-i & \left\{\bar{A}_{2}^{\prime} e^{i\left(\bar{k}_{2} \bar{x}-3 \bar{\omega} \bar{t}\right)}+\bar{B}_{2}^{\prime} e^{i\left[\left(\bar{k}_{0}+\bar{k}_{1}\right) \bar{x}-3 \bar{\omega} \bar{t}\right]}+e^{3 i\left(\bar{k}_{0} \bar{x}-\bar{\omega} \bar{t}\right)}\right\} \\
& +i\left\{\bar{A}_{2}^{\prime *} e^{-i\left(\bar{k}_{2}^{*} \bar{x}-3 \bar{\omega} \bar{t}\right)}+\bar{B}_{2}^{\prime *} e^{-i\left[\left(\bar{k}_{0}+\bar{k}_{1}\right)^{*} \bar{x}-3 \bar{\omega} \bar{t}\right]}+e^{-3 i\left(\bar{k}_{0}^{*} \bar{x}-\bar{\omega} \bar{t}\right)}\right\},
\end{aligned}
$$

or

$$
\begin{aligned}
32 i \bar{P}_{2}(\bar{x}, \bar{t})= & \bar{A}_{2}^{\prime} e^{i\left(\bar{k}_{2} \bar{x}-3 \bar{\omega} \bar{t}\right)}+\bar{B}_{2}^{\prime} e^{i\left[\left(\bar{k}_{0}+\bar{k}_{1}\right) \bar{x}-3 \bar{\omega} \bar{t}\right]}+e^{3 i\left(\overline{k_{0}} \bar{x}-\bar{\omega} \bar{t}\right)} \\
& -\bar{A}_{2}^{\prime *} e^{-i\left(\bar{k}_{2}^{*} \bar{x}-3 \bar{\omega} \bar{t}\right)}-\bar{B}_{2}^{\prime *} e^{-i\left[\left(\bar{k}_{0}+\bar{k}_{1}\right)^{*} \bar{x}-3 \bar{\omega} \bar{t}\right]}-e^{-3 i\left(\bar{k}_{0}^{*} \bar{x}-\bar{\omega} \bar{t}\right)},
\end{aligned}
$$

or

$$
\begin{aligned}
32 i \bar{P}_{2}(\bar{x}, \bar{t})=\bar{A}_{2}^{\prime} e^{-\bar{\beta}_{2} \bar{x}} e^{i\left(\bar{\alpha}_{2} \bar{x}-3 \bar{\omega} \bar{t}\right)}+\bar{B}_{2}^{\prime} e^{-\left(\bar{\beta}_{0}+\bar{\beta}_{1}\right) \bar{x}} e^{i\left[\left(\bar{\alpha}_{0}+\bar{\alpha}_{1}\right) \bar{x}-3 \bar{\omega} \bar{t}\right]} \\
+e^{-3 \bar{\beta}_{0} \bar{x}} e^{3 i\left(\bar{\alpha}_{0} \bar{x}-\bar{\omega} \bar{t}\right)}-\bar{A}_{2}{ }^{\prime *} e^{-\bar{\beta}_{2} \bar{x}} e^{-i\left(\bar{\alpha}_{2} \bar{x}-3 \bar{\omega} \bar{t}\right)} \\
-\bar{B}_{2}^{\prime *} e^{-\left(\bar{\beta}_{0}+\bar{\beta}_{1}\right) \bar{x}} e^{-i\left[\left(\bar{\alpha}_{0}+\bar{\alpha}_{1}\right) \bar{x}-3 \bar{\omega} \bar{t}\right]}-e^{-3 \bar{\beta}_{0} \bar{x}} e^{-3 i\left(\bar{\alpha}_{0} \bar{x}-\bar{\omega} \bar{t}\right)},
\end{aligned}
$$


Since $\bar{A}_{2}^{\prime}, \bar{B}_{2}^{\prime} \in \mathbb{C}$, we write $\bar{A}_{2}^{\prime}=\operatorname{Re} \bar{A}_{2}^{\prime}+i \operatorname{Im} \bar{A}_{2}^{\prime}$ and $\bar{B}_{2}^{\prime}=\operatorname{Re} \bar{B}_{2}^{\prime}+i \operatorname{Im} \bar{B}_{2}^{\prime}$. Then, $\bar{A}_{2}{ }^{*}=\operatorname{Re} \bar{A}_{2}^{\prime}-i \operatorname{Im} \bar{A}_{2}^{\prime}$ and $\bar{B}_{2}{ }^{\prime *}=\operatorname{Re} \bar{B}_{2}{ }^{\prime}-i \operatorname{Im} \bar{B}_{2}{ }^{\prime}$, and Equation (5.4.1) can be written as

$$
\begin{aligned}
& 32 i \bar{P}_{2}(\bar{x}, \bar{t})=\left(\operatorname{Re} \bar{A}_{2}^{\prime}+i \operatorname{Im} \bar{A}_{2}^{\prime}\right) e^{-\bar{\beta}_{2} \bar{x}} e^{i\left(\bar{\alpha}_{2} \bar{x}-3 \bar{\omega} \bar{t}\right)} \\
&+\left(\operatorname{Re} \bar{B}_{2}^{\prime}+i \operatorname{Im} \bar{B}_{2}^{\prime}\right) e^{-\left(\bar{\beta}_{0}+\bar{\beta}_{1}\right) \bar{x}} e^{i\left[\left(\bar{\alpha}_{0}+\bar{\alpha}_{1}\right) \bar{x}-3 \bar{\omega} \bar{t}\right]} \\
&-\left(\operatorname{Re} \bar{A}_{2}^{\prime}-i \operatorname{Im} \bar{A}_{2}^{\prime}\right) e^{-\bar{\beta}_{2} \bar{x}} e^{-i\left(\bar{\alpha}_{2} \bar{x}-3 \bar{\omega} \bar{t}\right)} \\
&-\left(\operatorname{Re} \bar{B}_{2}^{\prime}-i \operatorname{Im} \bar{B}_{2}^{\prime}\right) e^{-\left(\bar{\beta}_{0}+\bar{\beta}_{1}\right) \bar{x}} e^{-i\left[\left(\bar{\alpha}_{0}+\bar{\alpha}_{1}\right) \bar{x}-3 \bar{\omega} \bar{t}\right]} \\
&+e^{-3 \bar{\beta}_{0} \bar{x}} e^{3 i\left(\overline{\left.\alpha_{0} \bar{x}-\bar{\omega} \bar{t}\right)}-e^{-3 \bar{\beta}_{0} \bar{x}} e^{-3 i\left(\bar{\alpha}_{0} \bar{x}-\bar{\omega} \bar{t}\right)},\right.}
\end{aligned}
$$

or

$$
\begin{aligned}
32 i \bar{P}_{2}(\bar{x}, \bar{t})=\operatorname{Re} \bar{A}_{2}^{\prime} e^{-\bar{\beta}_{2} \bar{x}}\left[e^{i\left(\bar{\alpha}_{2} \bar{x}-3 \bar{\omega} \bar{t}\right)}-e^{-i\left(\bar{\alpha}_{2} \bar{x}-3 \bar{\omega} \bar{t}\right)}\right] \\
+i \operatorname{Im} \bar{A}_{2}^{\prime} e^{-\bar{\beta}_{2} \bar{x}}\left[e^{i\left(\bar{\alpha}_{2} \bar{x}-3 \bar{\omega} \bar{t}\right)}+e^{-i\left(\bar{\alpha}_{2} \bar{x}-3 \bar{\omega} \bar{t}\right)}\right] \\
+\operatorname{Re} \bar{B}_{2}^{\prime} e^{-\left(\bar{\beta}_{0}+\bar{\beta}_{1}\right) \bar{x}}\left\{e^{i\left[\left(\bar{\alpha}_{0}+\bar{\alpha}_{1}\right) \bar{x}-3 \bar{\omega} \bar{t}\right]}-e^{-i\left[\left(\bar{\alpha}_{0}+\bar{\alpha}_{1}\right) \bar{x}-3 \bar{\omega} \bar{t}\right]}\right\} \\
+i \operatorname{Im} \bar{B}_{2}^{\prime} e^{-\left(\bar{\beta}_{0}+\bar{\beta}_{1}\right) \bar{x}}\left\{e^{i\left[\left(\bar{\alpha}_{0}+\bar{\alpha}_{1}\right) \bar{x}-3 \bar{\omega} \bar{t}\right]}+e^{-i\left[\left(\bar{\alpha}_{0}+\bar{\alpha}_{1}\right) \bar{x}-3 \bar{\omega} \bar{t}\right]}\right\} \\
+e^{-3 \bar{\beta}_{0} \bar{x}} e^{3 i\left(\bar{\alpha}_{0} \bar{x}-\bar{\omega} \bar{t}\right)}-e^{-3 \bar{\beta}_{0} \bar{x}} e^{-3 i\left(\bar{\alpha}_{0} \bar{x}-\bar{\omega} \bar{t}\right)}
\end{aligned}
$$


or

$$
\begin{aligned}
& 32 \bar{P}_{2}(\bar{x}, \bar{t})=2 \operatorname{Re} \bar{A}_{2}^{\prime} e^{-\bar{\beta}_{2} \bar{x}}\left[\frac{e^{i\left(\bar{\alpha}_{2} \bar{x}-3 \bar{\omega} \bar{t}\right)}-e^{-i\left(\bar{\alpha}_{2} \bar{x}-3 \bar{\omega} \bar{t}\right)}}{2 i}\right] \\
& +2 \operatorname{Im} \bar{A}_{2}^{\prime} e^{-\bar{\beta}_{2} \bar{x}}\left[\frac{e^{i\left(\bar{\alpha}_{2} \bar{x}-3 \bar{\omega} \bar{t}\right)}+e^{-i\left(\bar{\alpha}_{2} \bar{x}-3 \bar{\omega} \bar{t}\right)}}{2}\right] \\
& +2 \operatorname{Re} \bar{B}_{2}^{\prime} e^{-\left(\bar{\beta}_{0}+\bar{\beta}_{1}\right) \bar{x}}\left\{\frac{e^{i\left[\left(\bar{\alpha}_{0}+\bar{\alpha}_{1}\right) \bar{x}-3 \bar{\omega} \bar{t}\right]}-e^{-i\left[\left(\bar{\alpha}_{0}+\bar{\alpha}_{1}\right) \bar{x}-3 \bar{\omega} \bar{t}\right]}}{2 i}\right\} \\
& +2 \operatorname{Im} \bar{B}_{2}^{\prime} e^{-\left(\bar{\beta}_{0}+\bar{\beta}_{1}\right) \bar{x}}\left\{\frac{e^{i\left[\left(\bar{\alpha}_{0}+\bar{\alpha}_{1}\right) \bar{x}-3 \bar{\omega} \bar{t}\right]}+e^{-i\left[\left(\bar{\alpha}_{0}+\bar{\alpha}_{1}\right) \bar{x}-3 \bar{\omega} \bar{t}\right]}}{2}\right\} \\
& +2 e^{-3 \bar{\beta}_{0} \bar{x}}\left[\frac{e^{3 i\left(\bar{\alpha}_{0} \bar{x}-\bar{\omega} \bar{t}\right)}-e^{-3 i\left(\bar{\alpha}_{0} \bar{x}-\bar{\omega} \bar{t}\right)}}{2 i}\right],
\end{aligned}
$$

or

$$
\begin{aligned}
16 \bar{P}_{2}(\bar{x}, \bar{t})=\operatorname{Re} \bar{A}_{2}^{\prime} e^{-\bar{\beta}_{2} \bar{x}} \sin \left(\bar{\alpha}_{2} \bar{x}-3 \bar{\omega} \bar{t}\right)+\operatorname{Im} \bar{A}_{2}^{\prime} e^{-\bar{\beta}_{2} \bar{x}} \cos \left(\bar{\alpha}_{2} \bar{x}-3 \bar{\omega} \bar{t}\right) \\
+\operatorname{Re} \bar{B}_{2}^{\prime} e^{-\left(\bar{\beta}_{0}+\bar{\beta}_{1}\right) \bar{x}} \sin \left[\left(\bar{\alpha}_{0}+\bar{\alpha}_{1}\right) \bar{x}-3 \bar{\omega} \bar{t}\right] \\
+\operatorname{Im} \bar{B}_{2}^{\prime} e^{-\left(\bar{\beta}_{0}+\bar{\beta}_{1}\right) \bar{x}} \cos \left[\left(\bar{\alpha}_{0}+\bar{\alpha}_{1}\right) \bar{x}-3 \bar{\omega} \bar{t}\right] \\
+e^{-3 \bar{\beta}_{0} \bar{x}} \sin 3\left(\bar{\alpha}_{0} \bar{x}-\bar{\omega} \bar{t}\right) .
\end{aligned}
$$


Thus

$$
\begin{aligned}
\bar{P}_{2}(\bar{x}, \bar{t})= & \frac{e^{-\bar{\beta}_{2} \bar{x}}}{16}\left[\operatorname{Re} \bar{A}_{2}^{\prime} \sin \left(\bar{\alpha}_{2} \bar{x}-3 \bar{\omega} \bar{t}\right)+\operatorname{Im} \bar{A}_{2}^{\prime} \cos \left(\bar{\alpha}_{2} \bar{x}-3 \bar{\omega} \bar{t}\right)\right] \\
& +\frac{e^{-\left(\bar{\beta}_{0}+\bar{\beta}_{1}\right) \bar{x}}}{16} \operatorname{Re} \bar{B}_{2}^{\prime} \sin \left[\left(\bar{\alpha}_{0}+\bar{\alpha}_{1}\right) \bar{x}-3 \bar{\omega} \bar{t}\right] \\
& +\frac{e^{-\left(\bar{\beta}_{0}+\bar{\beta}_{1}\right) \bar{x}}}{16} \operatorname{Im} \bar{B}_{2}^{\prime} \cos \left[\left(\bar{\alpha}_{0}+\bar{\alpha}_{1}\right) \bar{x}-3 \bar{\omega} \bar{t}\right] \\
& +\frac{e^{-3 \bar{\beta}_{0} \bar{x}}}{16} \sin 3\left(\bar{\alpha}_{0} \bar{x}-\bar{\omega} \bar{t}\right) .
\end{aligned}
$$

\subsection{Low-Frequency Limiting Behavior}

In the low-frequency region, we have from Equation (3.2.6) and Equation (3.2.7),

$$
\bar{\alpha}_{n}(\bar{\omega})=\bar{\beta}_{n}(\bar{\omega})=\sqrt{\frac{(n+1) \bar{\omega}}{2}}
$$

which gives $\bar{\alpha}_{0}(\bar{\omega})=\bar{\beta}_{0}(\bar{\omega})=\sqrt{\frac{\bar{\omega}}{2}}, \bar{\alpha}_{1}(\bar{\omega})=\bar{\beta}_{1}(\bar{\omega})=\sqrt{\bar{\omega}}$ and

$$
\bar{k}_{0} \cdot \bar{k}_{1}=\left(\sqrt{\frac{\bar{\omega}}{2}}+i \sqrt{\frac{\bar{\omega}}{2}}\right)(\sqrt{\bar{\omega}}+i \sqrt{\bar{\omega}})=i\left(\frac{\bar{\omega}}{\sqrt{2}}+\frac{\bar{\omega}}{\sqrt{2}}\right)=i \frac{2 \bar{\omega}}{\sqrt{2}}=i \sqrt{2} \bar{\omega},
$$


Then, from Equations (2.6.11) and (2.6.12), we have

$$
\begin{gathered}
\operatorname{Re} \bar{A}_{2}^{\prime}=-\frac{4 \bar{\omega}^{2}-(3-\sqrt{2}) \sqrt{2}}{4 \bar{\omega}^{2}+2}, \\
\operatorname{Im} \bar{A}_{2}^{\prime}=-\frac{6 \bar{\omega}}{4 \bar{\omega}^{2}+2}
\end{gathered}
$$

and

$$
\begin{gathered}
\operatorname{Re} \bar{B}_{2}^{\prime}=-\frac{3 \sqrt{2}}{4 \bar{\omega}^{2}+2} \\
\operatorname{Im} \bar{B}_{2}^{\prime}=\frac{6 \bar{\omega}}{4 \bar{\omega}^{2}+2}
\end{gathered}
$$

Expanding $\bar{P}$ into its real and imaginary parts, adding it to its complex conjugate and simplifying, the low-frequency approximation is given by $\bar{P}^{L F}=\bar{P}_{0}^{L F}+\varepsilon \bar{P}_{1}^{L F}+\varepsilon^{2} \bar{P}_{2}^{L F}$, where the terms $\bar{P}_{0}^{L F}, \varepsilon \bar{P}_{1}^{L F}$ and $\varepsilon^{2} \bar{P}_{2}{ }^{L F}$ are given by

$$
\begin{gathered}
\bar{P}_{0}^{L F}(\bar{x}, \bar{t})=-e^{-\sqrt{\frac{\omega}{2}} \bar{x}} \sin \left(\sqrt{\frac{\bar{\omega}}{2}} \bar{x}-\bar{\omega} \bar{t}\right) \\
\varepsilon \bar{P}_{1}^{L F}(\bar{x}, \bar{t})=-\frac{\varepsilon}{4}\left[e^{-\sqrt{\bar{\omega}} \bar{x}} \cos (\sqrt{\bar{\omega}} \bar{x}-2 \bar{\omega} \bar{t})-e^{-\sqrt{2 \bar{\omega}} \bar{x}} \cos (\sqrt{2 \bar{\omega}} \bar{x}-2 \bar{\omega} \bar{t})\right] \\
\varepsilon^{2} \bar{P}_{2}^{L F}(\bar{x}, \bar{t})=-\frac{\varepsilon^{2}}{16} \cdot \frac{A+B+C}{4 \bar{\omega}^{2}+2},
\end{gathered}
$$


where

$$
\begin{gathered}
A=\left\{\left[4 \bar{\omega}^{2}-(3-\sqrt{2}) \sqrt{2}\right] \sin \left(\sqrt{\frac{3 \bar{\omega}}{2}} \bar{x}-3 \bar{\omega} \bar{t}\right)+6 \bar{\omega} \cos \left(\sqrt{\frac{3 \bar{\omega}}{2}} \bar{x}-3 \bar{\omega} \bar{t}\right)\right\} e^{-\sqrt{\frac{3 \bar{\omega}}{2}} \bar{x}} \\
B=[-6 \bar{\omega} \cos (s \bar{x}-3 \bar{\omega} \bar{t})+3 \sqrt{2} \sin (s \bar{x}-3 \bar{\omega} \bar{t})] e^{-s \bar{x}} \\
C=-\left(4 \bar{\omega}^{2}+2\right) \sin \left[3\left(\sqrt{\frac{\bar{\omega}}{2} \bar{x}}-\bar{\omega} \bar{t}\right)\right] e^{-3 \sqrt{\frac{\bar{\omega}}{2}}},
\end{gathered}
$$

and

$$
s=\frac{1+\sqrt{2}}{\sqrt{2}} \bar{\omega}
$$

Figure 8 shows a comparison between the perturbation solution $\bar{P}=\overline{\mathcal{P}}+$ c.c. and the corresponding low-frequency approximation. From this figure, it is clear that the approximation is in excellent agreement with the corresponding perturbation solution in the low-frequency region. 


$$
\bar{P}=\overline{\mathcal{P}}+\text { c.c. }
$$

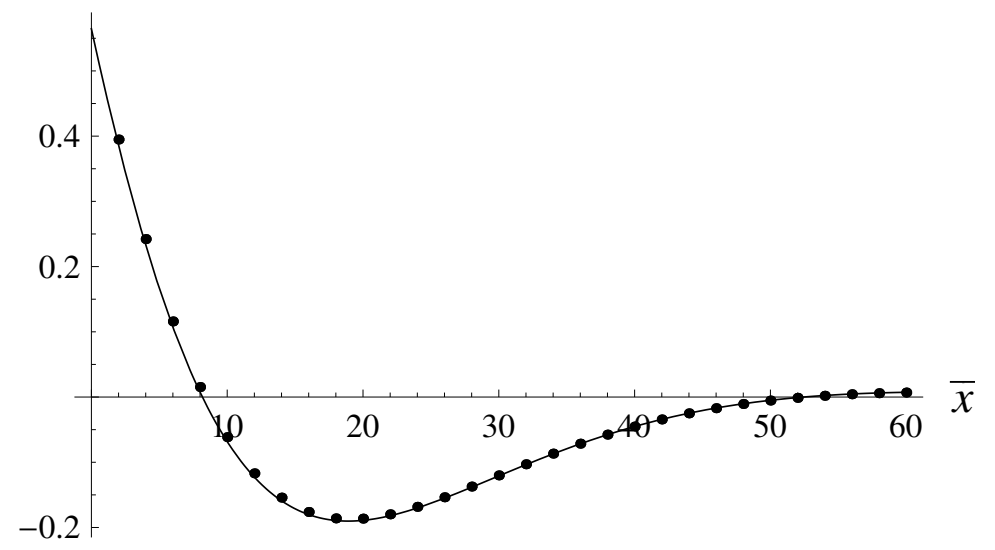

Figure 8. A comparison of the low-frequency $\bar{\omega}=0.01$ perturbation solution $\bar{P}=\overline{\mathcal{P}}+$ c.c. with the lowfrequency approximation $\bar{P}^{L F}$ for $\varepsilon=0.4 . \bar{P}$ is shown as a solid curve and the points are obtained from $\bar{P}^{L F}$. 


\subsection{High-Frequency Limiting Behavior}

In the high-frequency region, we have from Equation (3.2.8) and Equation (3.2.9),

$$
\bar{\alpha}_{n}(\bar{\omega})=(n+1) \bar{\omega}
$$

and

$$
\bar{\beta}_{n}(\bar{\omega})=\frac{1}{2}
$$

which gives $\bar{\alpha}_{0}(\bar{\omega})=\bar{\omega}, \bar{\alpha}_{1}(\bar{\omega})=2 \bar{\omega}, \bar{\beta}_{0}(\bar{\omega})=\bar{\beta}_{1}(\bar{\omega})=\frac{1}{2}$ and

$$
\bar{k}_{0} \cdot \bar{k}_{1}=\left(\sqrt{\frac{\bar{\omega}}{2}}+i \sqrt{\frac{\bar{\omega}}{2}}\right)(\sqrt{\bar{\omega}}+i \sqrt{\bar{\omega}})=i\left(\frac{\bar{\omega}}{\sqrt{2}}+\frac{\bar{\omega}}{\sqrt{2}}\right)=i \frac{2 \bar{\omega}}{\sqrt{2}}=i \sqrt{2} \bar{\omega},
$$

Then, from Equations (2.6.11) and (2.6.12), we have

$$
\begin{gathered}
\operatorname{Re} \bar{A}_{2}^{\prime}=-\frac{-\frac{9}{4} \bar{\omega}^{2}+\frac{1}{16}}{\frac{9}{4} \bar{\omega}^{2}+\frac{1}{16}} \\
\operatorname{Im} \bar{A}_{2}^{\prime}=-\frac{\frac{3}{4} \bar{\omega}}{\frac{9}{4} \bar{\omega}^{2}+\frac{1}{16}}
\end{gathered}
$$


and

$$
\begin{aligned}
& \operatorname{Re} \bar{B}_{2}^{\prime}=-\frac{\frac{9}{2} \bar{\omega}^{2}}{\frac{9}{4} \bar{\omega}^{2}+\frac{1}{16}} \\
& \operatorname{Im} \bar{B}_{2}^{\prime}=\frac{\frac{3}{4} \bar{\omega}}{\frac{9}{4} \bar{\omega}^{2}+\frac{1}{16}}
\end{aligned} .
$$

In the high-frequency region, using the high-frequency approximations for $\bar{\alpha}_{n}$ and $\bar{\beta}_{n}$, Equations (4.3.1), expanding $\bar{P}$ into its real and imaginary parts, adding it to its complex conjugate and simplifying, the high-frequency approximation is given by $\bar{P}^{H F}=\bar{P}_{0}{ }^{H F}+\varepsilon \bar{P}_{1}^{H F}+\varepsilon^{2} \bar{P}_{2}^{H F}$, where the terms $\bar{P}_{0}{ }^{H F}, \varepsilon \bar{P}_{1}^{H F}$ and $\varepsilon^{2} \bar{P}_{2}{ }^{H F}$ are given by

$$
\begin{gathered}
\bar{P}_{0}^{H F}(\bar{x}, \bar{t})=-e^{-\frac{\bar{x}}{2}} \sin \bar{\omega}(\bar{x}-\bar{t}) \\
\varepsilon \bar{P}_{1}^{H F}(\bar{x}, \bar{t})=-\frac{\varepsilon}{4} e^{-\frac{\bar{x}}{2}}\left(1-e^{-\frac{\bar{x}}{2}}\right) \cos 2 \bar{\omega}(\bar{x}-\bar{t}) \\
\varepsilon^{2} \bar{P}_{2}^{H F}(\bar{x}, \bar{t})=\frac{\varepsilon^{2}}{16} \cdot \frac{1}{\frac{1}{16}+\frac{9}{4} \bar{\omega}^{2}} e^{-\frac{\bar{x}}{2}}[D \cos 3 \bar{\omega}(\bar{x}-\bar{t})+E \sin 3 \bar{\omega}(\bar{x}-\bar{t})],
\end{gathered}
$$

where

$$
\begin{gathered}
D=-\frac{3}{4} \bar{\omega}+\frac{3}{4} \bar{\omega} e^{-\frac{\bar{x}}{2}} \\
E=-\left[\frac{1}{16}-\frac{9}{4} \bar{\omega}^{2}\right]-\frac{9}{2} \bar{\omega}^{2} e^{-\frac{\bar{x}}{2}}+\left[\frac{1}{16}+\frac{9}{4} \bar{\omega}^{2}\right] e^{-\bar{x}} .
\end{gathered}
$$


Figure 9 shows a comparison between the perturbation solution $\bar{P}=\overline{\mathcal{P}}+$ c.c. and the corresponding high-frequency approximation.. Again, we see that the approximation is in excellent agreement with the corresponding perturbation solution in the high-frequency regions.

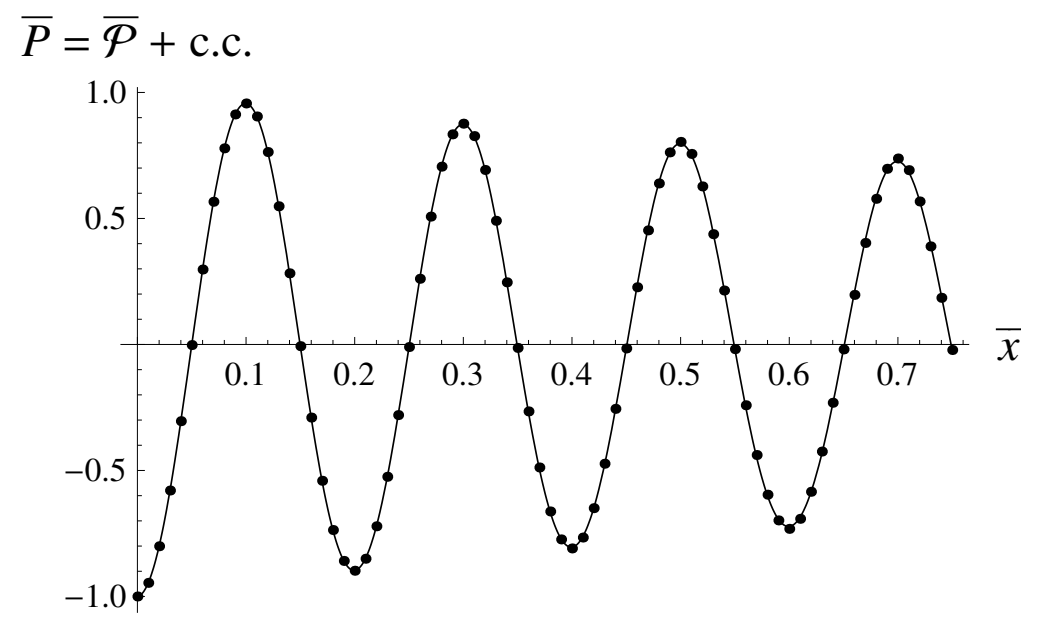

Figure 9. A comparison of the high-frequency $\bar{\omega}=10 \pi$ perturbation solution $\bar{P}=\overline{\mathcal{P}}+$ c.c. with the highfrequency approximation $\bar{P}^{H F}$ for $\varepsilon=0.4 . \bar{P}$ is shown as a solid curve and the points are obtained from $\bar{P}^{H F}$. 


\section{Chapter 6. Comparison of the Perturbation Solution with the Finite- Difference Solution}

\subsection{Overview}

In Chapter 2, the solution $\bar{P}$ of BVP was found using the perturbation method and in Chapter 4, convergence of the solution was verified by determining a value of the perturbation parameter, $\varepsilon_{\max }$, such that $\bar{P}$ remained bounded. However, $\bar{P}$ as determined is only valid after enough time has passed for the transient effects to die down. We shall now compare $\bar{P}$ with the solution of the non-dimensional form of BVP (1.7.1) as obtained using a nonstandard finitedifference scheme [19], which we denote by $\bar{\rho}$. Since $\bar{\rho}$ is valid for all times, we shall seek a time $\bar{T}$ large enough for any transient effects to become negligible. Then for $\bar{t} \geq \bar{T}, \bar{\rho} \approx \bar{P}$.

To determine $\bar{\rho}$, we will use the explicit scheme as outlined by Jordan and Mickens in [19]. Here, the continuous variables $\bar{x}$ and $\bar{t}$ are replaced by discrete sets of equally spaced points $\bar{x}_{m}$ and $\bar{t}_{k}$, where $\bar{x}_{m}=m \Delta \bar{x}$ and $\bar{t}_{k}=k \Delta \bar{t}$, respectively, where $m$ and $k$ are nonnegative integers. The corresponding pressure will then be denoted by $\bar{P}_{m}^{k}$. The derivatives are then discretized as follows:

$$
\bar{P}_{m}^{k+1}=\frac{2 \bar{P}_{m}^{k}-\bar{P}_{m}^{k-1}-\frac{\Delta \bar{t}}{2}\left(\varepsilon \bar{P}_{m}^{k}-1\right) \bar{P}_{m}^{k-1}-R^{2}\left(\bar{P}_{m+1}^{k}-2 \bar{P}_{m}^{k}+\bar{P}_{m-1}^{k}\right)}{1-\frac{\Delta \bar{t}}{2}\left(\varepsilon \bar{P}_{m}^{k}-1\right)},
$$

where, $R=\Delta \bar{t} / \Delta \bar{x}$. To ensure proper convergence, we must have $R<1$. This is satisfied in [19] by taking $\Delta \bar{x}=2 \Delta \bar{t}$. We will use the same $R$; however, we shall seek an optimum mesh size by varying the total number of nodal points over one quarter of a wavelength. 
Since the finite-difference scheme involves a boundary at some point $0<\bar{L}<\infty$, the effect of the presence of this boundary must be taken into consideration when comparing the two solution methods. We note that since the units are non-dimensional and the speed of the wave is normalized to unity, the spatial and temporal variables are equivalent. Thus, finding the time $\bar{T}$ at which the approximations are close is equivalent to finding a position $\bar{L}$ for the right boundary such that reflection from the boundary becomes negligible.

\subsection{Demonstrating the Convergence of the Finite-Difference Scheme Over One Quarter-Wavelength}

We shall now determine an optimal mesh size for the finite-difference scheme. Specifically, we will solve Equation (xmaxequation) over one quarter of a wavelength for increasingly smaller values of $\Delta \bar{t}$ until convergence occurs to the third decimal place. Table 2 gives the values of $\bar{\rho}(\bar{L}-\Delta \bar{x}, \bar{T})$ for several values of $\Delta \bar{t}^{-1}$ and the corresponding values of $\Delta \bar{x}^{-1}$ 


\begin{tabular}{|c|c|c|}
\hline$\Delta \bar{t}^{-1}$ & $\Delta \bar{x}^{-1}$ & $\bar{\rho}(\bar{L}-\Delta \bar{x}, \bar{T})$ \\
\hline \hline 625 & 312.5 & 0.105945262321074 \\
\hline 1250 & 625 & 0.0349286893647188 \\
\hline 2500 & 1250 & 0.0170705086266031 \\
\hline 3000 & 1500 & 0.0141534335150195 \\
\hline 3500 & 1750 & 0.0120916075568926 \\
\hline 3775 & 1887.5 & 0.0111914850660859 \\
\hline 3780 & 1890 & 0.0111770506623238 \\
\hline
\end{tabular}

Table 2. Illustrating the Convergence of One Quarter-Wavelength of the Finite-Difference Approximation $\bar{\rho}$ by Computing $\bar{\rho}$ at One Spatial Increment Before the Boundary at $\bar{L}$ for Decreasing Mesh Sizes. Here, $\bar{\omega}=10 \pi$ and $\varepsilon=0.4$.

Here, it is evident that $\bar{\rho}(\bar{L}-\Delta \bar{x}, \bar{T}) \rightarrow 0$ as $\Delta \bar{t} \rightarrow 0$ (or, $\Delta \bar{t}^{-1} \rightarrow \infty$ ).

\subsection{Determining the Position of the Right Boundary such that the Perturbation}

\section{Solution Decays to $10 \%$}

In this section, we shall determine a value of $\bar{L}$ such that the amplitude of $\bar{P}$ decays in amplitude to $10 \%$ of its value at source of the disturbance. To do this, we will compute $\bar{P}$ one quarter-wavelength to the left of the right boundary at $\bar{L} \cdot \bar{P}(\bar{L}-\lambda / 4, \bar{T})$ for boundaries placed at several odd multiples of quarter wavelengths are given in Table 3. 


\begin{tabular}{|c|c|c|}
\hline $\bar{L}=\bar{T}$ & $\bar{L}-\lambda / 4$ & $\bar{P}(\bar{L}-\lambda / 4, \bar{T})$ \\
\hline \hline 0.15 & 0.1 & 0.9558962911 \\
\hline 0.25 & 0.2 & 0.9135396006 \\
\hline 0.35 & 0.3 & 0.8728775809 \\
\hline$\vdots$ & $\vdots$ & $\vdots$ \\
\hline 4.55 & 4.5 & 0.1156853701 \\
\hline 4.65 & 4.6 & 0.1101040369 \\
\hline 4.75 & 4.7 & 0.1047891584 \\
\hline 4.85 & 4.8 & 0.0997282801 \\
\hline 4.95 & 4.9 & 0.09490950482 \\
\hline 5.15 & 5.1 & 0.0859533238 \\
\hline 5.35 & 5.3 & 0.07783573618 \\
\hline 5.55 & 5.5 & 0.07047940385 \\
\hline
\end{tabular}

Table 3. Determining the location at which the amplitude of $\bar{P}$ decays to $10 \%$ of its value at the signal in the high-frequency region. Here, $\bar{\omega}=10 \pi$ and $\varepsilon=0.4$.

In Table 3, the location at which the amplitude of the perturbation solution decays to ten percent is shown in bold face. This corresponds to ninety-seven quarter-wavelengths. For the computation of the data, $\bar{\omega}=10 \pi$ and $\varepsilon=0.4$.

\subsection{Using the Finite-Difference Solution to Establish the Validity of the}

\section{Perturbation Solution}

Here we wish to establish the validity of the perturbation solution $\bar{P}$. To do this, we shall compare $\bar{P}$ with the finite-difference solution $\bar{\rho}$, whose convergence is demonstrated over one quarter-wavelength in Section 6.2. Due to the presence of a right boundary $\bar{L}$ in the finitedifference and the corresponding boundary condition at $\bar{L}$; namely, the pressure must vanish at $\bar{L}$. 
Since we are most concerned with the high-frequency regime, we choose a frequency $\bar{\omega}=10 \pi$, which satisfies this condition. Furthermore, for the perturbation solution, we shall take the perturbation parameter to be $\varepsilon=0.4<\varepsilon_{\max }$ determined in Chapter 4. For the chosen frequency, we choose $\bar{L}=97 \lambda / 4$, where $\lambda$ is the wavelength of the pressure wave. This location is chosen so that the condition that the amplitude of the signal drops to $10 \%$ of its value at $\bar{x}=0$. The finite-difference solution is then computed for this $\bar{L}$.

The evolution of this solution is then compared with the evolution of the perturbation solution for times $\bar{t}$ such that the signal has reached one, five, seventeen and thirty-five quarterwavelengths and is shown in Figure 10. Figure 11 shows the evolution of the perturbation solution for ninety-seven quartet-wavelengths. In each of these figures, it is readily seen that the two solutions are in excellent agreement. Figure 11 is repeated in Figure 12, with the linear part of the perturbation, $\bar{P}_{0}$, superimposed on the plot for positions to the right of $\bar{L}$. Here, we see that when the solution is evolved to the point where the signal amplitude drops to $10 \%$, the perturbation solution $\bar{P}$ may be replaced with $\bar{P}_{0}$. 

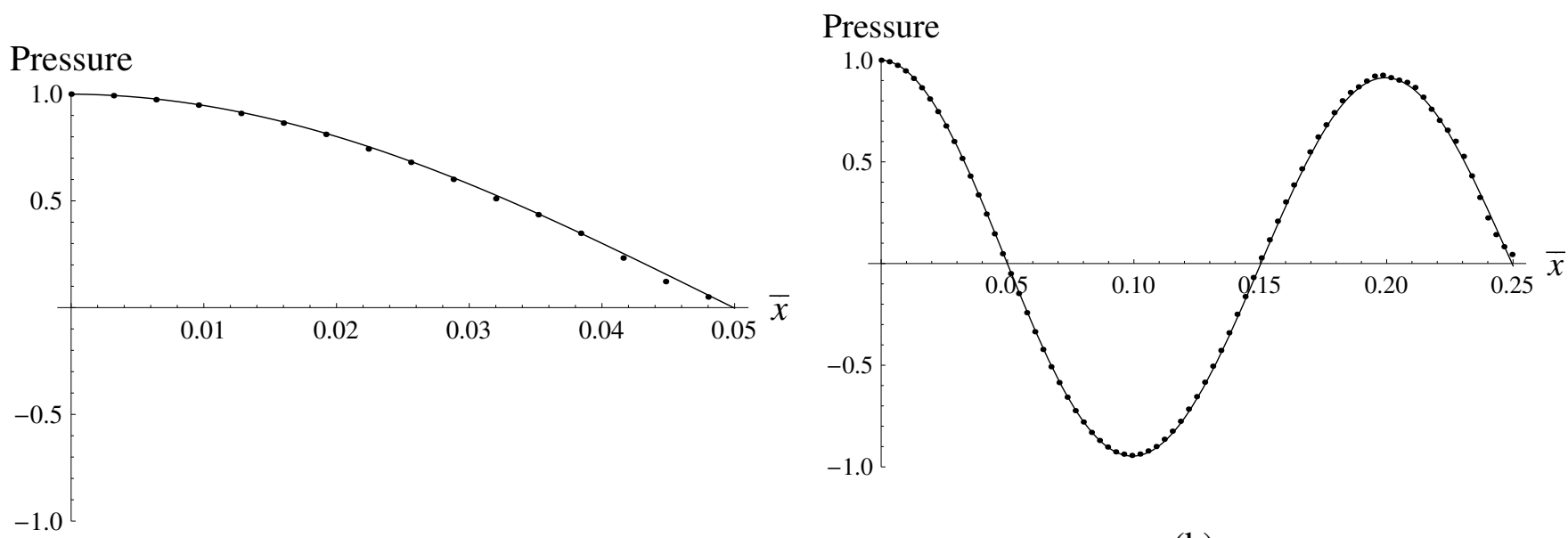

(b)

(a)

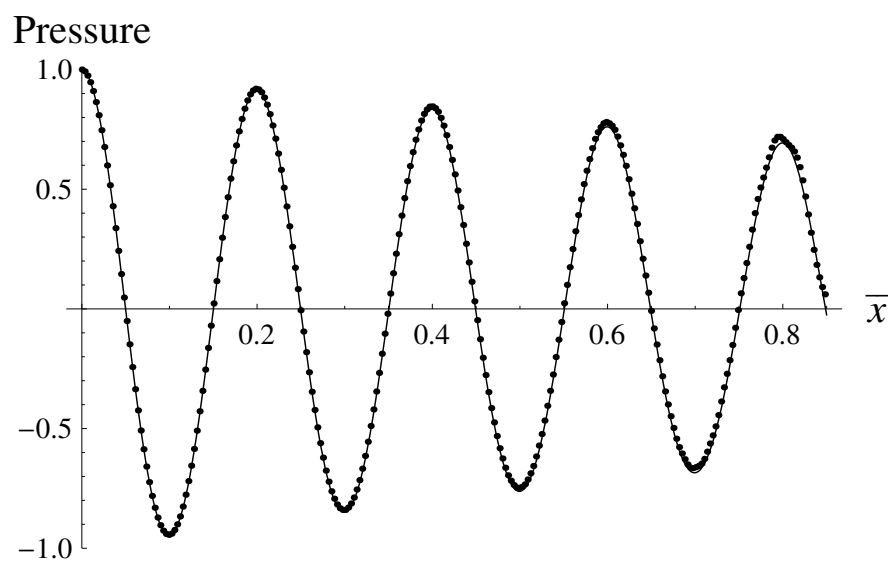

(c)
Pressure

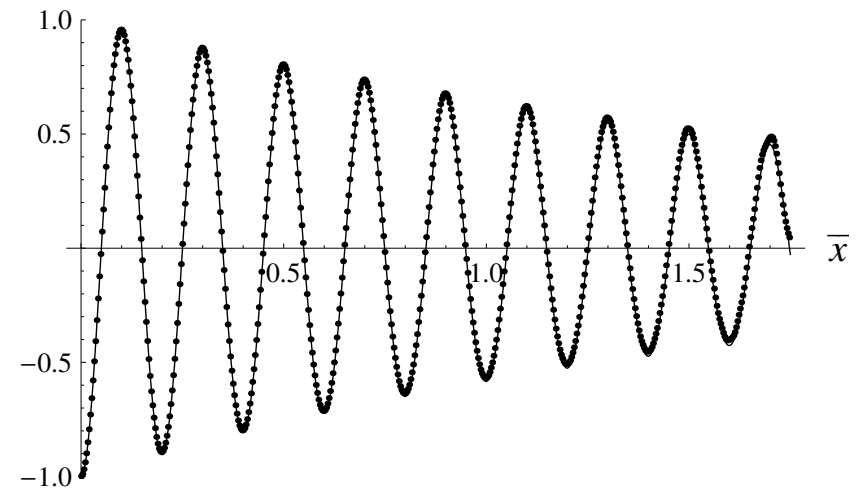

(d)

Figure 10. A comparison of the evolution of the perturbation solution $\bar{P}$ (solid) with the finite-difference solution $\bar{\rho}$ (dots) for (a) one, (b) five, (c) seventeen, and (d) thirty-five quarter-wavelengths with $\bar{\omega}=10 \pi$ and $\varepsilon=0.4$. The right boundary $\bar{L}$ used in the calculation of $\bar{\rho}$ is located at 97 quarterwavelengths. 


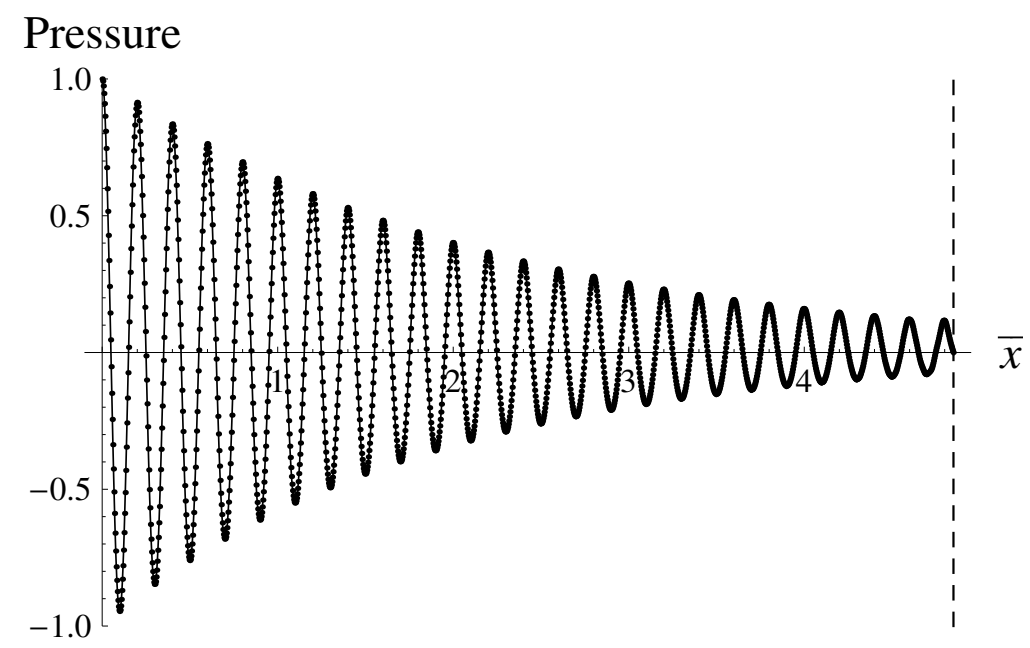

Figure 11. A comparison of the evolution of the perturbation solution $\bar{P}$ (solid) with the finite-difference solution $\bar{\rho}$ (dots) for ninety-seven quarter-wavelengths with $\bar{\omega}=10 \pi$ and $\varepsilon=0.4$. The right boundary $\bar{L}$ used in the calculation of $\bar{\rho}$ is located at 97 quarter-wavelengths.

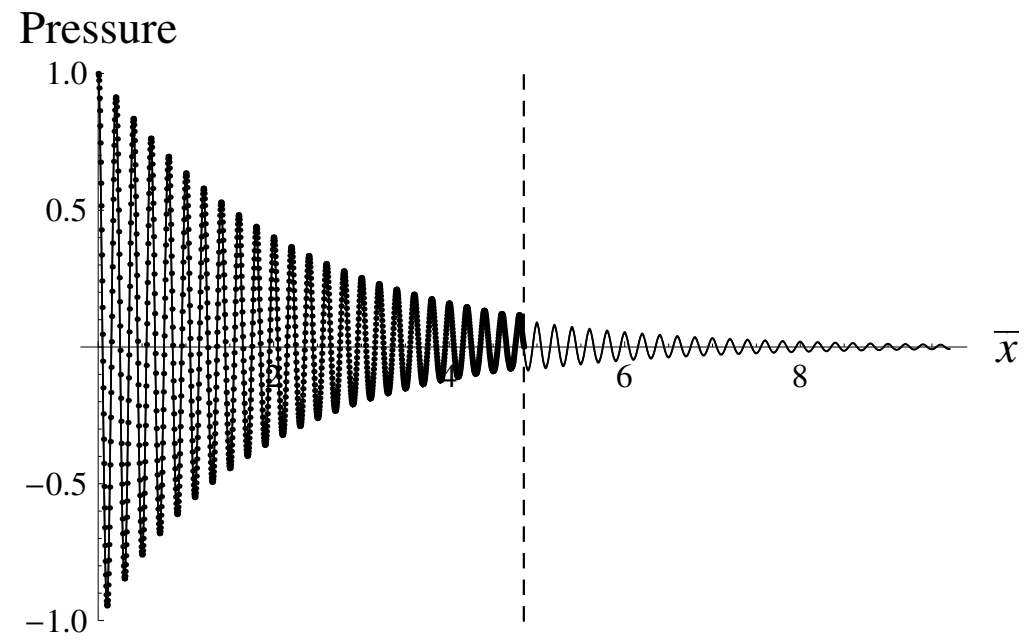

Figure 12. Demonstrating the validity of replacing the finite-difference solution $\bar{\rho}$ (dots) with the linear part of the perturbation solution $\bar{P}_{0}$ (dots) for ninety-seven quarter-wavelengths with $\bar{\omega}=10 \pi$ and $\varepsilon=0.4$. The right boundary $\bar{L}$ used in the calculation of $\bar{\rho}$ is located at 97 quarter-wavelengths. Note that the perturbation solution $\bar{P}$ is shown as a solid line on the left side of $\bar{L}$. 


\section{Chapter 7. Closure}

\subsection{Conclusions}

In this dissertation, the perturbation method is used to find the long-time solution, $\bar{P}=\overline{\mathcal{P}}+$ c.c. , to Equation (2.3.2). The dispersion relations are determined and their real and imaginary parts obtained. Particular attention is paid to the behavior of the dispersion relations in the low and high frequency regions. The wave speeds are analyzed in a similar manner. Next, we examine the behavior of the first-order correction term, $\bar{P}_{0}$, by investigating how the location of the maximum value of its modulus, $\bar{x}_{\max }$, varies in relation to frequency. This is done by computing its spatial derivative and determining where it vanishes. Exact solutions are found for the high and low frequency regimes. Numerical results are obtained for frequencies between these two regimes. A maximum value of the perturbation parameter, $\varepsilon_{\max }$, was then determined which ensures boundedness of the solution. Finally, a numerical solution, $\bar{\rho}$, was obtained using a non-standard finite-difference scheme. $\bar{\rho}$ was compared with $\bar{P}$ at a time $\bar{T}$. Moreover, the location of the right boundary $(\bar{x}=\bar{L}$ ) was determined so that the amplitude of $\bar{\rho}$ decays to $10 \%$ of its value at the signal location. Furthermore, we show that for this value of $\bar{L}$, the solution can be replaced by the linear part of the perturbation solution $\bar{P}_{0}$. The results of our investigations are summarized below. 
1. The moduli $\left|\overline{\mathcal{P}_{1}}\right|$ and $\left|\overline{\mathcal{P}_{2}}\right|$, the moduli of the first and second-order corrections, respectively, are bounded and decay to zero as $\bar{x} \rightarrow \infty .\left|\overline{\mathcal{P}_{1}}\right|$ and $\left|\overline{\mathcal{P}_{2}}\right|$ are shown plotted for several frequencies in Figure 1 and Figure 2, respectively. From these figures, it can be seen that $\left|\overline{\mathcal{P}_{1}}\right|$ and $\left|\overline{\mathcal{P}_{2}}\right|$ have unique maxima, that shift to the left as the frequency increases and to the right as the frequency is reduced.

2. The dispersion relations are determined in Chapter 3 (Figures $3 \mathrm{a}$ and $3 \mathrm{~b}$ ). Here, we find that for low frequencies, both the real and imaginary parts of the wavenumber are approximately equal and proportional to $\sqrt{\bar{\omega}}$, indicating that the medium assumes a diffusive-like nature in this frequency regime [16]; whereas, for high frequencies, the real part of the wavenumber is proportional to $\bar{\omega}$, while the imaginary part is approximately equal to the real constant 0.5 , indicating that the medium behaves similarly to one exhibiting relaxation behavior in this frequency regime $[12,28]$. For combustion to be supported, the medium must behave similarly to one exhibiting relaxation in a given frequency regime. Thus, we see that combustion is not supported for low frequencies.

3. The phase speeds are determined in Chapter 3 (Figure 4). Here, we find that each mode of the phase speed is monotonically increasing and bounded, approaching a constant value in the high-frequency limit. Moreover, we see that for all modes, $\bar{v}_{\mathrm{ph}, n}<1$ for all $\bar{\omega}$ 
4. In Chapter 4 , the location of the peak of $\left|\overline{\mathcal{P}_{1}}\right|, \bar{x}_{\max }$, is first determined from Equation (4.2.9). Next, the asymptotic behavior is determined analytically and numerically in low and high frequency limits. $\bar{x}_{\max }$ is shown to be asymptotic to the curve $2(\sqrt{2}-1) / \sqrt{\bar{\omega}}$ in the low-frequency limit and to the constant value $2 \ln 2$ in the high-frequency limit. Figure 5 shows a plot of $\bar{x}_{\max }$ versus $\bar{\omega}$. The limiting behavior is shown as dashed lines.

5. Bounds are obtained on the perturbation parameter $\varepsilon$ and a maximum value of $\varepsilon_{\max }=0.8$ is determined which ensures boundedness of the solution. As shown in Figure 6 , this value of $\varepsilon$ guarantees that both ratios, $\ln \left(\left|\overline{\mathcal{P}_{1}}\right| /\left|\overline{\mathcal{P}_{0}}\right|\right)$ and $\ln \left(\left|\overline{\mathcal{P}_{2}}\right| /\left|\overline{\mathcal{P}_{0}}\right|\right)$ remain much smaller than unity, thereby ensuring convergence of $\bar{P}$. Specifically, we find that $\varepsilon \leq 0.8$. Thus, for the numerical work, we use $\varepsilon=0.4$, well below the maximum value $\varepsilon_{\max }$.

6. In Chapter 5, analytic expressions are obtained for the solutions $\bar{P}_{0}, \bar{P}_{1}$ and $\bar{P}_{2}$. Approximations for these expressions are then obtained in the limiting cases of low and high frequency. The total approximate solutions $\bar{P}^{L F}$ and $\bar{P}^{H F}$ are then compared with $\bar{P}$ in the corresponding frequency regimes in Figures 8 and 9, respectively, where the agreement is seen to be excellent. In these figures, the dots are obtained from the approximations while the solid line is obtained from the general expression.

7. In Chapter 6, the perturbation method is compared with a positivity and boundedness preserving non-standard finite-difference scheme [18, 22]. First, convergence of the finite-difference solution $\bar{\rho}$ is established for one quarter wavelength by computing $\bar{\rho}$ at 
one spatial-step to the left of the right boundary for several decreasing mesh sizes, $(\Delta t, \Delta x)$. This is done since the $\mathrm{BC}$ demands that $\bar{\rho}$ must vanish at the right boundary, so that the value of $\bar{\rho}$ one spatial step to the left must tend to zero as the mesh size decreases. The results for several mesh sizes are given in Table 2. From these values, it can be readily seen that $\bar{\rho}(\bar{L}-\Delta \bar{x}, \bar{T})$ decreases to zero as the mesh size is reduced.

8. Since the perturbation method is valid only for long times (after transient effects have dissipated), a position for the right boundary, $\bar{L}$, is established such that enough time has passed for the long-time behavior to become dominant. Specifically, the location of the right boundary is chosen so that $\bar{P}$ decays to approximately $10 \%$ of its value at the signal source, as shown in Figure 11. This ensures that reflections from the boundary will be negligible. The evolution of the finite-difference solution, $\bar{\rho}$, compared to the perturbation solution, $\bar{P}$, is shown in Figure 10 for one quarter-wavelength, five quarterwavelengths, seventeen quarter-wavelengths, and thirty-five quarter-wavelengths. Here, the decay of the amplitude of $\bar{\rho}$ as $\bar{x}$ approaches $\bar{L}$ can clearly be seen.

\subsection{Limitations}

The method described in this paper has some limitations. The solution obtained from the perturbation method is valid only for long times, after initial transient effects have dissipated; whereas, the solution obtained from the finite-difference scheme is valid for all times. Thus, the two solutions can only be legitimately compared for large times. For small times, the comparison becomes invalid. 
Another limitation is due to the presence of the right boundary in the finite-difference scheme. In order to avoid any contributions from reflections at the boundary, the boundary must be taken far from the source. However, this presents a computational challenge due to the large number of points needed in the finite-difference calculations.

The efficiency of the finite-difference scheme is strongly dependent on the mesh size. The number of calculations required for a given mesh size may require a large amount of time allocated to the computation and be impractical.

One way to overcome some of these limitations is illustrated in Figure 11. Here, the pressure wave is plotted versus $\bar{x}$ at time $\bar{T}=4.85$, over the interval $[0,2 \bar{L}]$, where $\bar{L}=\bar{T}$, $\bar{\omega}=10 \pi$ and $\varepsilon=0.4<\varepsilon_{\max }$. These values of the parameters are chosen so that the amplitude of the pressure wave decays to about $10 \%$ of its value at the applied signal. To the left of $\bar{L}$, the plot of the finite-difference solution $(\bar{\rho})$ is shown. However, to the right of $\bar{L}$, the plot of the corresponding linear part $\bar{P}_{0}$ is shown. From this plot, it can be seen that for points sufficiently far from the signal, $\bar{x}>\bar{L}$, the solution can be replaced by the linear part of the pressure wave, $\bar{P}_{0}$ 


\subsection{Connections to Other Fields}

Equation (1.6.1) can be used to model many phenomena in areas other than gas combustion. In fact, many of these phenomena occur in fields outside of the physical sciences: for example, in biology [23] and population genetics [2]. In each case, the physical parameters and constraints will be specific to the discipline, as well as the interpretation of the solution. We have presented a non-dimensional form of Equation (1.6.1) for gas combustion in Equation (2.3.2). Thus, the non-dimensional solution can be obtained and the parameters modified accordingly. 


\section{Appendix A. Mathematica Code for the Finite-Difference Calculation}

\section{Initializations}

SetDirectory["C:\Dissertation Manuscript"];

Off[General::"spell1"]; Off[FindRoot::"cvmit"]; Off[FindRoot::"srect"]; Off[Power::"infy"];

Off[œ::"indet"]; Off[General::"stop"]; Off[FindRoot::"lstol"];

Off[\$RecursionLimit::"reclim"]; Off[Graphics::"gprim"]; Off[MakeExpression::"boxfmt"];

$\varepsilon=.4$

$\omega=10 . \pi$

$\lambda=2 \pi / \omega ; \mathrm{n}=97 ; \mathrm{L}=\mathrm{n} \lambda / 4 ; \gamma=-1 ; \alpha=1 . ; \mathrm{dt}=1 . / 625 ; \mathrm{dx}=2 \mathrm{dt} ; \mathrm{R}=\mathrm{dt} / \mathrm{dx} ;$

$\mathrm{M}=$ Floor$[\mathrm{L} / \mathrm{dx}+1]$; 
$\bar{T}=\lambda / 4$

$\mathrm{T}=\lambda / 4 ; \mathrm{K}=$ Floor $[\mathrm{T} / \mathrm{dt}+1] ;$ Mprime $=\mathrm{N}[\mathrm{T} / \mathrm{dx}] ;$ FinalState $=\{\} ;$

filename $=$ StringJoin[ToString[T/( $\lambda / 4)]$, "quarterwavelengths.dat"];

$\mathrm{S}=$ Table $[0,\{\mathrm{t}, 0, \mathrm{~T}, \mathrm{dt}\},\{\mathrm{x}, 0, \mathrm{~L}, \mathrm{dx}\}]$

Do $[\mathrm{S}[[\mathrm{k}, 1]]=\mathrm{N}[\operatorname{Sin}[(\mathrm{k}-1) \mathrm{dt} \omega]],\{\mathrm{k}, 1, \mathrm{~K}\}]$;

$\operatorname{Do}[\mathrm{S}[[\mathrm{k}, \mathrm{m}]]$

$=\left(R^{2}(S[[k-1, m+1]]-2 S[[k-1, m]]+S[[k-1, m-1]])+(2 S[[k-1, m]]-S[[k-2, m]])-1 / 2 \gamma d t(\alpha-\right.$

$\varepsilon \mathrm{S}[[\mathrm{k}-1, \mathrm{~m}]]) \mathrm{S}[[\mathrm{k}-2, \mathrm{~m}]]) /(1-1 / 2 \gamma \mathrm{dt}(\alpha-\varepsilon \mathrm{S}[[\mathrm{k}-1, \mathrm{~m}]]))$,

$\{\mathrm{k}, 3, \mathrm{~K}\},\{\mathrm{m}, 2, \mathrm{M}-1\}]$

$\operatorname{Do}[\{\mathrm{y}=(\mathrm{m}-1) \mathrm{dx} ; \mathrm{s}=\mathrm{S}[[\mathrm{K}, \mathrm{m}]] ;$ FinalState $=\operatorname{Join}[$ FinalState, $\{\{\mathrm{y}, \mathrm{s}\}\}]\}$,

$\{\mathrm{m}, 1$, Mprime+1 $\}$;

$\mathrm{S}=$.

Export[filename, FinalState]; 
$\bar{T}=5 \lambda / 4$

$\mathrm{T}=5 \lambda / 4 ; \mathrm{K}=$ Floor $[\mathrm{T} / \mathrm{dt}+1] ;$ Mprime $=\mathrm{N}[\mathrm{T} / \mathrm{dx}] ;$ FinalState $=\{\} ;$

filename $=$ StringJoin[ToString[T/(入/4)],"quarterwavelengths.dat"];

$\mathrm{S}=\operatorname{Table}[0,\{\mathrm{t}, 0, \mathrm{~T}, \mathrm{dt}\},\{\mathrm{x}, 0, \mathrm{~L}, \mathrm{dx}\}]$

Do $[\mathrm{S}[[\mathrm{k}, 1]]=\mathrm{N}[\operatorname{Sin}[(\mathrm{k}-1) \mathrm{dt} \omega]],\{\mathrm{k}, 1, \mathrm{~K}\}]$;

$\operatorname{Do}[\mathrm{S}[[\mathrm{k}, \mathrm{m}]]$

$=\left(R^{2}(S[[k-1, m+1]]-2 S[[k-1, m]]+S[[k-1, m-1]])+(2 S[[k-1, m]]-S[[k-2, m]])-1 / 2 \gamma d t(\alpha-\right.$

$\varepsilon \mathrm{S}[[\mathrm{k}-1, \mathrm{~m}]]) \mathrm{S}[[\mathrm{k}-2, \mathrm{~m}]]) /(1-1 / 2 \gamma \mathrm{dt}(\alpha-\varepsilon \mathrm{S}[[\mathrm{k}-1, \mathrm{~m}]]))$,

$\{\mathrm{k}, 3, \mathrm{~K}\},\{\mathrm{m}, 2, \mathrm{M}-1\}]$

$\operatorname{Do}[\{\mathrm{y}=(\mathrm{m}-1) \mathrm{dx} ; \mathrm{s}=\mathrm{S}[[\mathrm{K}, \mathrm{m}]]$; FinalState $=\operatorname{Join}[$ FinalState, $\{\{\mathrm{y}, \mathrm{s}\}\}]\}$,

$\{\mathrm{m}, 1$, Mprime+1 $\}]$

$\mathrm{S}=$.

Export[filename, FinalState]; 
$\bar{T}=17 \lambda / 4$

$\mathrm{T}=17 \lambda / 4 ; \mathrm{K}=$ Floor $[\mathrm{T} / \mathrm{dt}+1] ;$ Mprime $=\mathrm{N}[\mathrm{T} / \mathrm{dx}] ;$ FinalState $=\{\} ;$

filename = StringJoin[ToString[T/(入/4)],"quarterwavelengths.dat"];

$\mathrm{S}=$ Table $[0,\{\mathrm{t}, 0, \mathrm{~T}, \mathrm{dt}\},\{\mathrm{x}, 0, \mathrm{~L}, \mathrm{dx}\}]$;

Do $[\mathrm{S}[[\mathrm{k}, 1]]=\mathrm{N}[\operatorname{Sin}[(\mathrm{k}-1) \mathrm{dt} \omega]],\{\mathrm{k}, 1, \mathrm{~K}\}]$

$\operatorname{Do}[\mathrm{S}[[\mathrm{k}, \mathrm{m}]]$

$=\left(R^{2}(S[[k-1, m+1]]-2 S[[k-1, m]]+S[[k-1, m-1]])+(2 S[[k-1, m]]-S[[k-2, m]])-1 / 2 \gamma d t(\alpha-\right.$

$\varepsilon \mathrm{S}[[\mathrm{k}-1, \mathrm{~m}]]) \mathrm{S}[[\mathrm{k}-2, \mathrm{~m}]]) /(1-1 / 2 \gamma \mathrm{dt}(\alpha-\varepsilon \mathrm{S}[[\mathrm{k}-1, \mathrm{~m}]]))$,

$\{\mathrm{k}, 3, \mathrm{~K}\},\{\mathrm{m}, 2, \mathrm{M}-1\}]$

$\operatorname{Do}[\{\mathrm{y}=(\mathrm{m}-1) \mathrm{dx} ; \mathrm{s}=\mathrm{S}[[\mathrm{K}, \mathrm{m}]] ;$ FinalState $=\operatorname{Join}[$ FinalState, $\{\{\mathrm{y}, \mathrm{s}\}\}]\}$,

$\{\mathrm{m}, 1$, Mprime+1 $\}]$

$S=$

Export[filename, FinalState]; 
$\bar{T}=35 \lambda / 4$

$\mathrm{T}=35 \lambda / 4 ; \mathrm{K}=$ Floor$[\mathrm{T} / \mathrm{dt}+1] ;$ Mprime $=\mathrm{N}[\mathrm{T} / \mathrm{dx}] ;$ FinalState $=\{\} ;$

filename $=$ StringJoin[ToString[T/(入/4)],"quarterwavelengths.dat"];

$\mathrm{S}=\operatorname{Table}[0,\{\mathrm{t}, 0, \mathrm{~T}, \mathrm{dt}\},\{\mathrm{x}, 0, \mathrm{~L}, \mathrm{dx}\}]$

Do $[\mathrm{S}[[\mathrm{k}, 1]]=\mathrm{N}[\operatorname{Sin}[(\mathrm{k}-1) \mathrm{dt} \omega]],\{\mathrm{k}, 1, \mathrm{~K}\}]$;

$\operatorname{Do}[\mathrm{S}[[\mathrm{k}, \mathrm{m}]]$

$=\left(R^{2}(S[[k-1, m+1]]-2 S[[k-1, m]]+S[[k-1, m-1]])+(2 S[[k-1, m]]-S[[k-2, m]])-1 / 2 \gamma d t(\alpha-\right.$

$\varepsilon \mathrm{S}[[\mathrm{k}-1, \mathrm{~m}]]) \mathrm{S}[[\mathrm{k}-2, \mathrm{~m}]]) /(1-1 / 2 \gamma \mathrm{dt}(\alpha-\varepsilon \mathrm{S}[[\mathrm{k}-1, \mathrm{~m}]]))$,

$\{\mathrm{k}, 3, \mathrm{~K}\},\{\mathrm{m}, 2, \mathrm{M}-1\}]$;

$\operatorname{Do}[\{\mathrm{y}=(\mathrm{m}-1) \mathrm{dx} ; \mathrm{s}=\mathrm{S}[[\mathrm{K}, \mathrm{m}]] ;$ FinalState $=$ Join[FinalState, $\{\{\mathrm{y}, \mathrm{s}\}\}]\}$,

$\{\mathrm{m}, 1$, Mprime+1 $\}]$

$\mathrm{S}=$.

Export[filename, FinalState]; 
$\bar{T}=97 \lambda / 4$

$\mathrm{T}=\mathrm{L} ; \mathrm{K}=$ Floor $[\mathrm{T} / \mathrm{dt}+1] ;$ Mprime $=\mathrm{N}[\mathrm{T} / \mathrm{dx}] ;$ FinalState $=\{\} ;$

filename $=$ String Join[ToString[T/( $\lambda / 4)]$, "quarterwavelengths.dat"];

$\mathrm{S}=\operatorname{Table}[0,\{\mathrm{t}, 0, \mathrm{~T}, \mathrm{dt}\},\{\mathrm{x}, 0, \mathrm{~L}, \mathrm{dx}\}]$

Do $[S[[k, 1]]=N[\operatorname{Sin}[(k-1) d t \omega]],\{k, 1, K\}]$;

$\operatorname{Do}[\mathrm{S}[[\mathrm{k}, \mathrm{m}]]$

$=\left(R^{2}(S[[k-1, m+1]]-2 S[[k-1, m]]+S[[k-1, m-1]])+(2 S[[k-1, m]]-S[[k-2, m]])-1 / 2 \gamma d t(\alpha-\right.$

$\varepsilon \mathrm{S}[[\mathrm{k}-1, \mathrm{~m}]]) \mathrm{S}[[\mathrm{k}-2, \mathrm{~m}]]) /(1-1 / 2 \gamma \mathrm{dt}(\alpha-\varepsilon \mathrm{S}[[\mathrm{k}-1, \mathrm{~m}]]))$,

$\{\mathrm{k}, 3, \mathrm{~K}\},\{\mathrm{m}, 2, \mathrm{M}-1\}]$

$\operatorname{Do}[\{\mathrm{y}=(\mathrm{m}-1) \mathrm{dx} ; \mathrm{s}=\mathrm{S}[[\mathrm{K}, \mathrm{m}]] ;$ FinalState $=$ Join[FinalState, $\{\{\mathrm{y}, \mathrm{s}\}\}]\}$,

$\{\mathrm{m}, 1$, Mprime+1 $\}]$

$\mathrm{S}=$.

Export[filename,FinalState]; 


\section{Appendix B. Mathematica Code for Computing the Location of the Peak of the First-Order Correction}

\section{Initializations}

SetDirectory ["C : \dissertation" ];

Off [ FindRoot :: "1stol" ];

$\alpha\left[\mathrm{n}_{-}\right.$Integer, $\mathrm{w}_{-}$Real $]:=\sqrt{\frac{(n+1) \omega}{2}} \sqrt{\sqrt{(n+1)^{2} \omega^{2}+1}+(n+1) \omega} ;$

$\beta\left[\mathrm{n}_{-}\right.$Integer, $\mathrm{w}_{-}$Real $]:=\sqrt{\frac{(n+1) \omega}{2}} \sqrt{\sqrt{(n+1)^{2} \omega^{2}+1}-(n+1) \omega} ;$

$\operatorname{modU1}\left[\omega \_\operatorname{Real}, \mathrm{x} \_\operatorname{Real}\right]:=\operatorname{Abs}\left[-\frac{1}{8}\left(e^{-\beta[1, \omega] \mathrm{x}} \mathrm{e}^{\mathrm{I} \alpha[1, \omega] \mathrm{x}}-\mathrm{e}^{-2 \beta[0, \omega] \mathrm{x}} \mathrm{e}^{2 \mathrm{I} \alpha[0, \omega] x}\right)\right]$;

$\mathrm{F}\left[\omega_{-}\right.$Real, $\mathrm{x}_{-}$Real $]:=\operatorname{modU} 1[\omega, \mathrm{x}]$

$\mathrm{G}\left[\omega_{-}\right.$Real, $\mathrm{x}_{-}$Real $]:=\partial_{\mathrm{u}} \mathrm{F}[\omega, \mathrm{u}] / .\{\mathrm{u} \rightarrow \mathrm{x}\}$ 


\section{Compute $\mathbf{x} \_\max$ for low frequencies.}

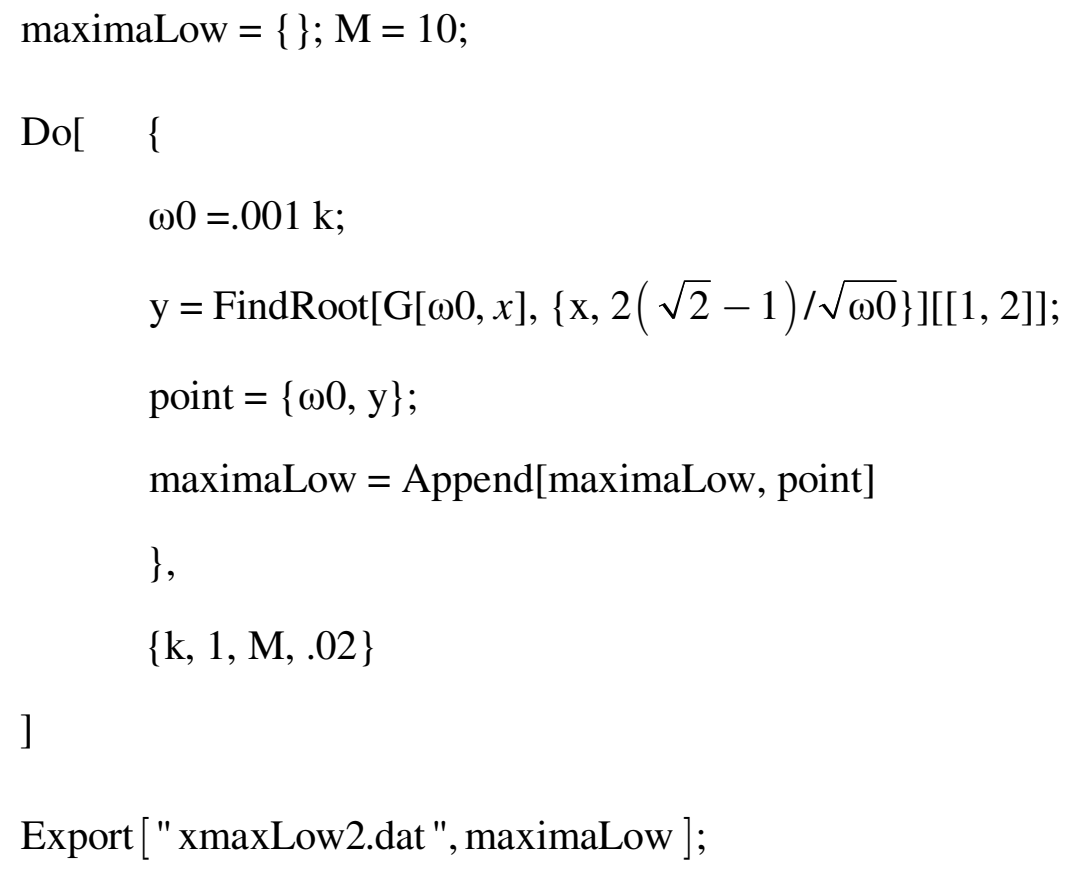

\section{Compute $\mathbf{x} \_\max$ for high frequencies.}

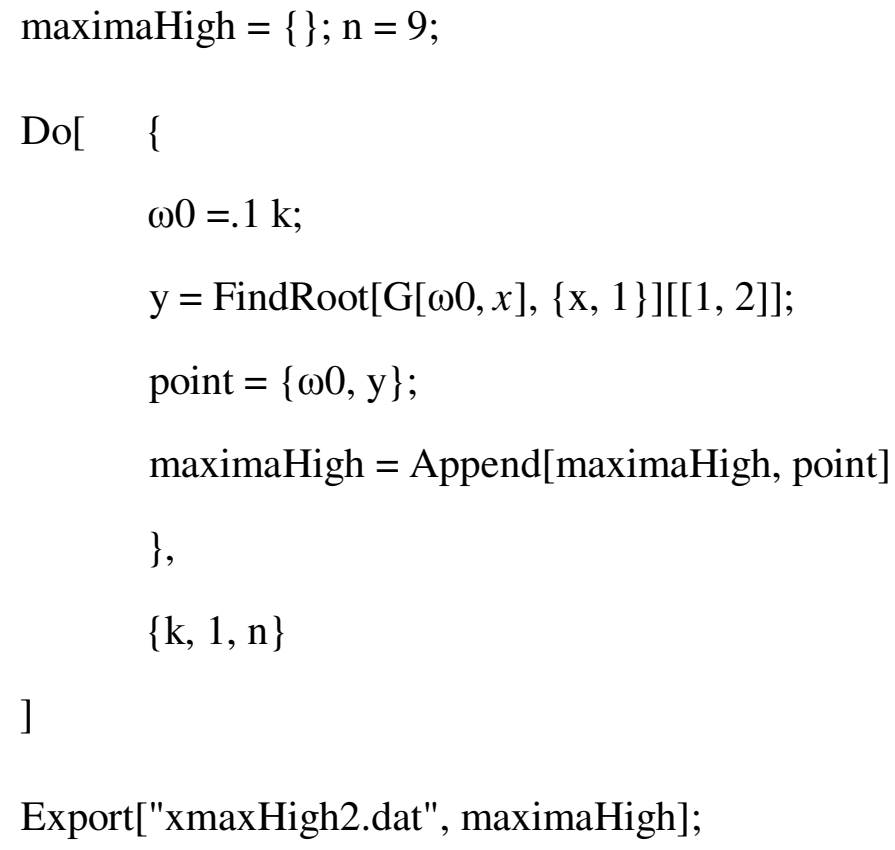




\section{References}

[1] G. B. Arfken and H. J. Weber, Mathematical methods for physicist, 4th edition, Academic Press, San Diego, CA, 1995.

[2] D. Aronson and H. Weinberger, Nonlinear diffusion in population genetics, combustion, and nerve pulse propagation, Partial Differential Equations and Related Topics (Jerome Goldstein, ed.), Lecture Notes in Mathematics, vol. 446, Springer Berlin / Heidelberg, 1975, 10.1007/BFb0070595, 5-49.

[3] J. H. Balbi, P.A. Santoni and J.L. Dupuy, "Dynamic modelling of fire spread across a fuel bed”, Int. J. Wildland Fire 9(4) (1999), 275-284

[4] J. H. Balbi, P.A. Santoni and J.L. Dupuy, "Dynamic modelling of upslope fire growth", Int. J. Wildland Fire 9(4) (1999), 285-292

[5] Boltzmann, L., Ableitung des stefan'schen gesetzes, betreffend die abhängigkeit der wärmestrahlung von der temperatur aus der electromagnetischen lichttheorie, Annalen der Physik und Chemie, Bd. 22 (1884), 291-294

[6] W. Chen, Rates of pyrolysis and combustion of bark by thermogravimetric analysis, Department of Chemical Engineering, Anderson Hall, University of

[7] P. K. Daripa and L. Sirovich, Exact and approximate gas dynamics using the tangent gas, J. Comp. Phys. 62 (1986), 400-413

[8] L. Debnath, An introduction to nonlinear partial differential equations for scientist and engineers, 2nd edition., Birkhauser, Boston, MA, 2004.

[9] J. Dupuy and M. Larini, Fire spread through a porous forest fuel bed: a radiative and convective model including fire-induced flow effects, Int. J. Wildland Fire 9(3) (1999), $155-172$

[10] S.J. Farlow, Partial differential equations for scientist and engineers, Dover, New York, NY, 1993.

[11] R. A. Fisher, The wave of advance of advantageous genes, Ann. Eugen. 7 (1937), 355-369. 
[12] F. Frachi and B. Straughan, Continuous dependence on the relaxation and modelling unbounded growth in theories of heat conduction with finite propagation speeds, J. Math. Anal. Appl. 185 (1994), 726-746.

[13] A. E. Green and P.M. Naghdi, A re-examination of the basic postulates of thermomechanics, Proc. Roy. Soc. A 432 (1991), 171-194.

[14] A.E. Green and P.M. Naghdi, Thermoelasticity without energy dissipation, Journal of Elasticity Springer Netherlands 31 (1993), 189-208

[15] M. E. Gurtin and A. C. Pipkin, A general theory of heat conduction with finite wave speeds, Arch. Rat. Mech. Anal. 31 (1968), 113-126.

[16] J. D. Jackson, Classical electrodynamics, 3rd edition, Wiley, New York, NY, 1999.

[17 ] P. M. Jordan and R. E. Mickens, A positivity-preserving nonstandard finite difference scheme for the dwe, Num. Meth. Partial Diff. Eqn 20 (2004), 639-649.

[18] P. M. Jordan and A. Puri, On the propagation of plane waves in type-III thermoelastic media, Proc. R. Soc. Lond. A 460 (2004), 3203-3221.

[19] A. Kolmogorov, I. Petrovski and N. Piskunov, A study of the diffusion equation with increase in the amount of substance, and its application to a biological problem, Bull. Univ. Moscow Ser. Int. A 11 (1937)

[20] J. Masoliver and G. Weiss, Telegrapher's equations with variable propagation speeds, Phys. Rev. E 49 (1994), 3852-3854

[21] M. J. Lighthill and G. B. Whitman, On kinematic waves ii. a theory of traffic flow on long crowded roads, Proc. R. Soc. A 229 (1955), 317-345.

[22] J.E. Macias-Diaz and A. Puri, A boundedness-preserving finite-difference scheme for a damped nonlinear wave equation, Appl. Numer. Math. 60 (2010), 934-948. 
[23] J. D. Murray, Mathematical biology, Springer, New York, NY, 1989.

[24] G. Rosen, Stability of pressure waves in a combustion field, Am. Rocket Soc. J. 30 (1960), $422-423$.

[25] G. Rosen, Nonlinear pressure oscillations in a combustion field, Am. Rocket Soc. J. 32 (1962).

[26] G. Rosen, Solutions of a certain nonlinear wave equation, J. Appl. Math. 44 (1966).

[27] K. R. Sharma, Damped wave transport and relaxation, Elsevier, San Diego, CA, 2005.

[28] J.G. Skellam, Random dispersal in theoretical populations, Biometrika 38 (1951), $196-218$

[29] Stefan, J.: Über die beziehung zwischen der wärmestrahlung und der temperatur, Sitzungsberichte der mathematisch-naturwissenschaftlichen Classe der kaiserlichen Akademie der Wissenschaften, Bd. 79 (1879), 391-428.

[30 ] H.C. Tuckwell, A study of some diffusion models of population growth, Theoretic. Pop. Biol. 5 (1974), 345-357

[31] H.F. Weinberger, On spreading speeds and traveling waves for growth and migration models in a periodic habitat, J. Math. Biol. 45 no. 2 (2007), 207-222

[32] F.A. Williams, Combustion theory ( $2^{\text {nd }}$ edition), Benjamin Cummins, 1985 Mississippi

[33] Q. Zhou, et. al., The ignition criterion of pulverized coal flow and its experimental verification, Challenges of Power Engineering and Environment Vol. 1, Springer, Berlin/Heidelberg (2007), 923-927

[34] Y. B. Zeldovich, et. al., The mathematical theory of combustion and explosions (New York: Consultants Bureau) (1985) 


\section{Vita}

John Eslick was born in Little Rock, Arkansas. He attended the University of New Orleans where he received a Bachelor of Science in Mathematics in 1997, minoring in Physics. He enrolled in the graduate school at the University of New Orleans, receiving a Master of Science in Mathematics in 1999. For the next five years, he taught at local high schools and community colleges in the New Orleans area. In 2004 he entered the Engineering and Applied Science Doctoral Program, in the Physics Department at the University of New Orleans under the advisement of Dr. Ashok Puri. 